T. Kajiwara, K. Kato and C. Nakayama

Nagoya Math. J.

Vol. 189 (2008), 63-138

\title{
LOGARITHMIC ABELIAN VARIETIES
}

\author{
TAKESHI KAJIWARA, KAZUYA KATO AND \\ CHIKARA NAKAYAMA
}

\section{Dedicated to Professor Luc Illusie}

\begin{abstract}
We develop the algebraic theory of log abelian varieties. This is Part II of our series of papers on log abelian varieties, and is an algebraic counterpart of the previous Part I ([6]), where we developed the analytic theory of $\log$ abelian varieties.
\end{abstract}

\section{Part II. Algebraic theory}

\section{Introduction}

This is Part II of our series of papers on log abelian varieties, and is an algebraic counterpart of the previous Part I ([6]), where we developed the analytic theory of $\log$ abelian varieties.

Degenerating abelian varieties can not preserve group structures, properness, and smoothness at the same time. However, in a log world, degenerating abelian varieties can become group objects called log abelian varieties which behave like proper smooth objects. For some background, see the introduction of Part I.

In Part I [6], we studied a complex analytic theory of log abelian varieties. In this Part II and sequel, we develop an algebraic theory of log abelian varieties.

Our philosophy, main ideas, and methods are illustrated in the introductory section, Section 1, by using Tate elliptic curves as examples. The main definitions and basic results concerning log abelian varieties are given in Sections 2-4. In Section 2 we introduce the notion $\log 1$-motif, and by using it, we define in Section 3 log abelian variety with constant degeneration which is a special case of $\log$ abelian variety. We define log abelian variety in Section 4. The proofs of the results are given in Sections 5-11.

Received September 27, 2005.

Revised December 20, 2006.

2000 Mathematics Subject Classification: Primary 14K10; Secondary 14J10, 14D06. 
In Sections 1-4, we refer to Sections 5-11 for almost all the proofs. (In the beginning of each section 1-4, we give a list of propositions whose proofs are put off and explain where we give their proofs.) The reason why we put the proofs in later sections is that the proofs of some results are very long and we wish that the reader can understand the main story early. A reader who prefers to know the proofs right after the results appear could read this paper in the following order: 1, 5, 2, 6, 3, 7, 8, 4 and 9-11.

In the sequel (Part III, etc.), one of the main subjects will be moduli spaces of $\log$ abelian varieties.

While we were writing this paper, we learned that V. Pahnke completed a beautiful work [12] on log abelian varieties. His formulation is different from ours: He works with the inverse limits of blowing ups along log structures. Log elliptic curves were already studied by M. C. Olsson in [11]. He also worked with the inverse limits of blowing ups along log structures.

Acknowledgments. A part of this work was done while the second author was a visitor of University of Minnesota in the spring of 1992 . He expresses his sincere gratitudes, especially to Professor W. Messing, for the hospitality and stimulating discussions. The authors are very much grateful to Professor Kazuhiro Fujiwara for enlightening discussions. They learned much from his unpublished work [3] on degeneration of abelian varieties.

\section{$\S 1$. Tate curves}

In this section, we explain our methods by using Tate curves as examples. The proof of Proposition 1.7 is given in Section 5 .

1.1. In this section, let $K$ be a complete discrete valuation field, let $q$ be a non-zero element of the maximal ideal $m_{K}$ of the valuation ring $O_{K}$ of $K$, and let $E_{q}$ be the Tate elliptic curve over $K$ with " $q$-invariant" $q([13]$, [14] Appendix C, Section 14).

We have a canonical isomorphism

$$
E_{q}(L) \cong L^{\times} / q^{\mathbb{Z}}
$$

for any finite extension $L$ of $K$.

We ask questions.

QUESTION 1. Is there a proper model of $E_{q}$ over $O_{K}$ to which the group structure of $E_{q}$ extends? 
The answer is "no" as is well known. We next ask

QUESTION 2. Is there a best model among all proper models of $E_{q}$ over $O_{K}$ ?

QUESTION 3. If the best proper model exists, and if $L$ is a finite extension of $K$, is the base change of the best model under $\operatorname{Spec}\left(O_{L}\right) \rightarrow \operatorname{Spec}\left(O_{K}\right)$ the best proper model of $E_{q} \otimes_{K} L$ over $O_{L}$ ?

It is difficult to have a natural formulation of the best model which gives affirmative answers to both the questions 2 and 3.

In fact, there is a unique minimal one among regular proper flat models of $E_{q}$ over $O_{K}$ (it is the model $\overline{\mathcal{G}}_{m}^{\pi} / q^{\mathbb{Z}}$ in [1] VII, which we review in 1.4-1.5 below, where $\pi$ is a prime element of $K$ ), but this construction does not commute with the base change $\operatorname{Spec}\left(O_{L}\right) \rightarrow \operatorname{Spec}\left(O_{K}\right)(\pi$ is not a prime element in $L$ if the ramification index of $L / K$ is $>1$ ).

In this paper, we show that all the questions $1,2,3$ have the answers "yes", if we look for the answers in the log world. As we will see, in the log world, $E_{q}$ extends to a log elliptic curve (=1-dimensional log abelian variety) $\mathcal{E}_{q}$ over $O_{K}$, which has a group structure. This construction commutes with the base change $\operatorname{Spec}\left(O_{L}\right) \rightarrow \operatorname{Spec}\left(O_{K}\right)$. This $\mathcal{E}_{q}$ plays the role of the best proper model of $E_{q}$ over $O_{K}$. Such a nice object does not exist in the world of the usual algebraic geometry.

1.2. For an fs log scheme $S$ (cf. [7], [10], [5] for basic terminology in log geometry in the sense of Fontaine-Illusie), let (fs $/ S$ ) be the category of fs $\log$ schemes over $S$. We regard $(\mathrm{fs} / S)$ as a site endowed with the usual étale topology. That is, a covering $\left(U_{i} \rightarrow U\right)_{i}$ of an object $U$ of $\left(\mathrm{fs}_{\mathrm{s}} / S\right)$ means a covering of the underlying scheme of $U$ by étale schemes $U_{i}$ over $U$ which are endowed with the inverse images of the $\log$ structure of $U$.

In our definition in Section 4, a $\log$ abelian variety over $S$ is a sheaf of abelian groups on $(\mathrm{fs} / S)$ satisfying certain conditions.

1.3. In this 1.3 , we describe one basic property 1.3 .1 of $\mathcal{E}_{q}$. In $1.4-1.5$, we consider some proper models of $E_{q}$ over $O_{K}$ which live in the world of schemes, and compare them with $\mathcal{E}_{q}$. In 1.6 , we give an explicit construction of $\mathcal{E}_{q}$.

In the rest of Section 1 , let $S=\operatorname{Spec}\left(O_{K}\right)$ with the canonical log structure (that is, the $\log$ structure defined by the closed point). Our $\mathcal{E}_{q}$ is a sheaf of abelian groups on $(\mathrm{fs} / S)$. Let $(\mathrm{fs} / S)^{\prime}$ be the full subcategory of 
(fs $/ S$ ) consisting of objects on which $m_{K}$ is locally nilpotent. We endow $(\mathrm{fs} / S)^{\prime}$ with the usual étale topology.

Define a commutative group sheaf $\mathbb{G}_{m, \log }$ and its subgroup sheaf $\mathbb{G}_{m, \log }^{(q)}$ on $(\mathrm{fs} / S)$ as follows: For an object $U$ of $(\mathrm{fs} / S)$,

$$
\begin{aligned}
& \mathbb{G}_{m, \log }(U)=\Gamma\left(U, M_{U}^{\mathrm{gp}}\right), \\
& \mathbb{G}_{m, \log }^{(q)}(U)=\left\{\varphi \in \Gamma\left(U, M_{U}^{\mathrm{gp}}\right) \mid \text { locally on } U, \text { there exist } i, j \in \mathbb{Z}\right. \\
& \text { such that } \left.q^{i}|\varphi| q^{j}\right\} .
\end{aligned}
$$

Here, $q \in M_{S} \subset \mathcal{O}_{S}$ is viewed as a section of $M_{U}^{\mathrm{gp}}$, and for local sections $a, b \in M_{U}^{\mathrm{gp}}, a \mid b$ means $a^{-1} b \in M_{U}$. (We write the monoid law of the logarithmic structure multiplicatively as in [7].) To see that $\mathbb{G}_{m, \log }^{(q)}$ is in fact a subgroup sheaf, let $\varphi, \varphi^{\prime} \in \Gamma\left(U, M_{U}^{\mathrm{gp}}\right)$ satisfy $q^{i}|\varphi| q^{j}$ and $q^{i^{\prime}}\left|\varphi^{\prime}\right| q^{j^{\prime}}$. Then $q^{i+i^{\prime}}\left|\varphi \varphi^{\prime}\right| q^{j+j^{\prime}}$ and $q^{-j}\left|\varphi^{-1}\right| q^{-i}$ so that the both $\varphi \varphi^{\prime}$ and $\varphi^{-1}$ belong to $\mathbb{G}_{m, \log }^{(q)}$.

1.3.1. The restriction of $\mathcal{E}_{q}$ to $(\mathrm{fs} / S)^{\prime}$ coincides with

$$
\mathbb{G}_{m, \log }^{(q)} / q^{\mathbb{Z}} .
$$

In particular, for a finite extension $L$ of $K$ and for $n \geq 1$, we have

$$
\mathcal{E}_{q}\left(O_{L} / m_{L}^{n}\right)=\left(\mathbb{G}_{m, \log }^{(q)} / q^{\mathbb{Z}}\right)\left(O_{L} / m_{L}^{n}\right)=\left(L^{\times} / U_{L}^{(n)}\right) / q^{\mathbb{Z}},
$$

where $\operatorname{Spec}\left(O_{L} / m_{L}^{n}\right)$ is endowed with the inverse image of the canonical log structure of $\operatorname{Spec}\left(O_{L}\right)$, and $U_{L}^{(n)}=\operatorname{Ker}\left(O_{L}^{\times} \rightarrow\left(O_{L} / m_{L}^{n}\right)^{\times}\right)$. The canonical isomorphism $E_{q}(L) \cong L^{\times} / q^{\mathbb{Z}}(1.1 .1)$ is obtained as

$$
E_{q}(L) \cong \mathcal{E}_{q}\left(O_{L}\right) \stackrel{\cong}{\lim _{n}} \mathcal{E}_{q}\left(O_{L} / m_{L}^{n}\right)={\underset{\lim }{n}}_{(}\left(L^{\times} / U_{L}^{(n)}\right) / q^{\mathbb{Z}}=L^{\times} / q^{\mathbb{Z}} .
$$

1.4. We now consider proper models of $E_{q}$ over $O_{K}$ and describe their relations with $\mathcal{E}_{q}$.

For a non-empty subset $I$ of $\mathbb{Q}$ which is stable under the translation by $\mathbb{Z}$ such that $I / \mathbb{Z}$ is finite, there exists a proper scheme $\mathcal{E}_{q}^{(I)}$ over $O_{K}$ such that $\mathcal{E}_{q}^{(I)} \otimes_{O_{K}} K=E_{q}$, having the characterizing property (1.4.1) below. For example, for a non-zero element $t$ of $m_{K}$ such that $\operatorname{ord}_{K}(t)$ divides $\operatorname{ord}_{K}(q)$, the model $\overline{\mathcal{G}}_{m}^{t} / q^{\mathbb{Z}}$ in [1] VII, 1.6 coincides with $\mathcal{E}_{q}^{((1 / d) \mathbb{Z})}$, where $d=\operatorname{ord}_{K}(q) / \operatorname{ord}_{K}(t)$. An explicit construction of the fs $\log$ scheme $\mathcal{E}_{q}^{(I)}$ is explained in 1.5. 
The scheme $\mathcal{E}_{q}^{(I)}$ has a natural fs log structure (see 1.4.1 below). As we will see in Section 5 , the sheaf $\operatorname{Mor}_{S}\left(, \mathcal{E}_{q}^{(I)}\right)$ on (fs/S), which we identify with $\mathcal{E}_{q}^{(I)}$ itself, is a subsheaf of $\mathcal{E}_{q}$. That is, various proper models are various subsheaves of $\mathcal{E}_{q}$.

For $\alpha \in I$, let $\alpha^{\prime}$ be the smallest element of the set $\{\beta \in I \mid \beta>\alpha\}$. Define a subsheaf $\mathbb{G}_{m, \log }^{(q, I)}$ of $\mathbb{G}_{m, \log }$ on $(\mathrm{fs} / S)$ as follows. For an object $U$ of $($ fs $/ S$ ),

$$
\begin{aligned}
& \mathbb{G}_{m, \log }^{(q, I)}(U)=\left\{\varphi \in \Gamma\left(U, M_{U}^{\mathrm{gp}}\right) \mid \text { locally on } U \text {, there exist } \alpha \in I\right. \text { and } \\
& \text { an integer } \left.k \geq 1 \text { such that } k \alpha, k \alpha^{\prime} \in \mathbb{Z} \text { and } q^{k \alpha}\left|\varphi^{k}\right| q^{k \alpha^{\prime}}\right\} \text {. }
\end{aligned}
$$

We have

$$
\mathbb{G}_{m, \log }^{(q, I)} \subset \mathbb{G}_{m, \log }^{(q)}, \quad \mathbb{G}_{m, \log }^{(q, I)} / q^{\mathbb{Z}} \subset \mathbb{G}_{m, \log }^{(q)} / q^{\mathbb{Z}} .
$$

Note that $\mathbb{G}_{m, \log }^{(q)}$ is a subgroup sheaf of $\mathbb{G}_{m, \log }$ as is explained in 1.3 , but $\mathbb{G}_{m, \log }^{(q, I)}$ is not a subgroup sheaf of $\mathbb{G}_{m, \log }$. For example, if $\varphi, \varphi^{\prime}$ are sections of $\mathbb{G}_{m, \log }^{(q, \mathbb{Z})}$ satisfying $1|\varphi| q$ and $1\left|\varphi^{\prime}\right| q$, then we have always $1\left|\varphi \varphi^{\prime}\right| q^{2}$, but we do not necessarily have $1\left|\varphi \varphi^{\prime}\right| q$ or $q\left|\varphi \varphi^{\prime}\right| q^{2}$.

For an object $W$ of (fs $/ S$ ), we identify $W$ with the sheaf

$$
(\mathrm{fs} / S) \longrightarrow(\operatorname{Sets}) ; U \longmapsto \operatorname{Mor}(U, W)
$$

represented by $W$. If the underlying scheme of $W$ is proper over $O_{K}$, the underlying scheme of $W$ is determined by the underlying schemes of $W \otimes_{O_{K}}$ $O_{K} / m_{K}^{n}(n \geq 1)([4])$ and hence determined by the restriction of the sheaf $W$ to $(\mathrm{fs} / S)^{\prime}$. Hence the following 1.4.1 characterizes the proper scheme $\mathcal{E}_{q}^{(I)}$ over $O_{K}$.

1.4.1. Define the log structure of $\mathcal{E}_{q}^{(I)}$ to be the subsheaf of the structural sheaf consisting of local sections whose restrictions to $E_{q}$ are invertible. Then this log structure of $\mathcal{E}_{q}^{(I)}$ is an fs log structure, and the restriction of $\mathcal{E}_{q}^{(I)}$ to $(\mathrm{fs} / S)^{\prime}$ coincides with

$$
\mathbb{G}_{m, \log }^{(q, I)} / q^{\mathbb{Z}}
$$

For example, if $t$ is a non-zero element of $m_{K}$ such that $\operatorname{ord}_{K}(t)$ divides $\operatorname{ord}_{K}(q)$, the restriction of $\overline{\mathcal{G}}_{m}^{t} / q^{\mathbb{Z}}\left(=\mathcal{E}_{q}^{((1 / d) \mathbb{Z})}\right.$ with $\left.d=\operatorname{ord}_{K}(q) / \operatorname{ord}_{K}(t)\right)$ to $(\mathrm{fs} / S)^{\prime}$ coincides with

$$
\left\{\varphi \in \mathbb{G}_{m, \log } \mid \text { locally, } t^{r}|\varphi| t^{r+1} \text { for some } r \in \mathbb{Z}\right\} / q^{\mathbb{Z}} .
$$

We describe further properties of $\mathcal{E}_{q}^{(I)}$. 
1.4.2. There is a natural bijection between $I / \mathbb{Z}$ and the set of all irreducible components of the special fiber of $\mathcal{E}_{q}^{(I)}$.

1.4.3. If $J$ is a non-empty subset of $I$ and is stable under the translation by $\mathbb{Z}$, there is a unique morphism

$$
\mathcal{E}_{q}^{(I)} \longrightarrow \mathcal{E}_{q}^{(J)}
$$

of models of $E_{q}$ whose restriction to (fs $\left./ S\right)^{\prime}$ is the evident inclusion map of sheaves. For $\alpha \in I \backslash J$, the irreducible component of the special fiber of $\mathcal{E}_{q}^{(I)}$ corresponding to $\alpha$ collapses into one point in the special fiber of $\mathcal{E}_{q}^{(J)}$. For $\alpha \in J$, the irreducible component of the special fiber of $\mathcal{E}_{q}^{(I)}$ corresponding to $\alpha$ maps onto the irreducible component of the special fiber of $\mathcal{E}_{q}^{(J)}$ corresponding to $\alpha$.

As a morphism of sheaves, $\mathcal{E}_{q}^{(I)} \rightarrow \mathcal{E}_{q}^{(J)}$ is injective, and not surjective if $I \neq J$. However the map of the underlying sets of schemes $\mathcal{E}_{q}^{(I)} \rightarrow \mathcal{E}_{q}^{(J)}$ is surjective, and not injective if $I \neq J$.

For example, we have diagrams in which the right square is commutative:

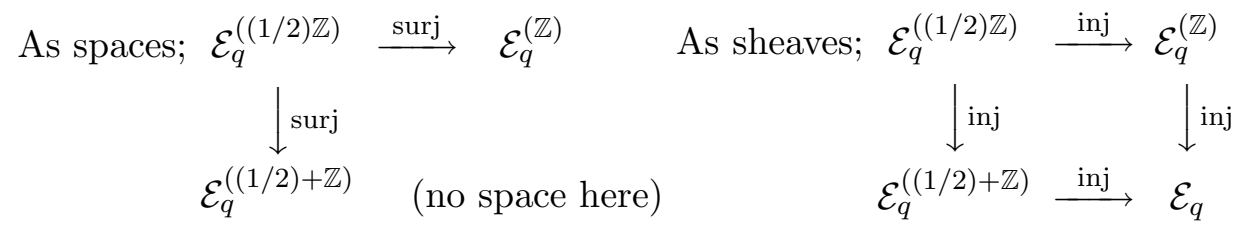

The special fiber of $\mathcal{E}_{q}^{((1 / 2) \mathbb{Z})}$ has two irreducible components as mentioned in (1.4.2). The irreducible component of the special fiber of $\mathcal{E}_{q}^{((1 / 2) \mathbb{Z})}$ corresponding to $1 / 2 \bmod \mathbb{Z}$ collapses into one point in the special fiber of $\mathcal{E}_{q}^{(\mathbb{Z})}$, and the other irreducible component of the special fiber of $\mathcal{E}_{q}^{((1 / 2) \mathbb{Z})}$ corresponding to $0 \bmod \mathbb{Z}$ collapses into one point in the special fiber of $\mathcal{E}_{q}^{((1 / 2)+\mathbb{Z})}$. Hence if there were a proper flat model of $E_{q}$ over $O_{K}$ which had morphisms from both $\mathcal{E}_{q}^{(\mathbb{Z})}$ and $\mathcal{E}_{q}^{((1 / 2)+\mathbb{Z})}$, the special fiber of such a model should be a one point set (because all irreducible components of the special fiber of $\mathcal{E}_{q}^{((1 / 2) \mathbb{Z})}$ should collapse in it), but this is impossible.

In the right diagram, $\mathcal{E}_{q}$ lives outside the world of schemes, and looks like a "true minimal model". We ask

QUESTION 4. What does this mean for the total picture of the theory of minimal models in algebraic geometry? 
For example, does such "true minimal model" outside the usual world of schemes appear in degenerations of $K 3$-surfaces? At present, we have no answer to Question 4.

1.5. We explain the construction of $\mathcal{E}_{q}^{(I)}$. We follow the method of Raynaud in [1] VII, Section 1, applying log geometry.

Let $n \geq 1$, and let $S_{n}=\operatorname{Spec}\left(O_{K} / m_{K}^{n}\right)$ which we endow with the inverse image of the canonical $\log$ structure of $S=\operatorname{Spec}\left(O_{K}\right)$. For $\alpha \in I$, let $V_{\alpha, n}$ be the subsheaf of $\mathbb{G}_{m, \log }$ on (fs $\left./ S_{n}\right)$ defined by

$$
\begin{array}{r}
V_{\alpha, n}(U)=\left\{\varphi \in \Gamma\left(U, M_{U}^{\mathrm{gp}}\right)\left|q^{k \alpha}\right| \varphi^{k} \mid q^{k \alpha^{\prime}} \text { for some integer } k \geq 1\right. \\
\text { such that } \left.k \alpha, k \alpha^{\prime} \in \mathbb{Z}\right\},
\end{array}
$$

where $\alpha^{\prime}$ is the smallest element of $I$ satisfying $\alpha^{\prime}>\alpha$. Let $e=\operatorname{ord}_{K}(q)$, and define an additive monoid $N_{\alpha}$ by

$$
N_{\alpha}=\left\{(a, b) \in \mathbb{Z}^{2} \mid a+\alpha e b \geq 0, a+\alpha^{\prime} e b \geq 0\right\} .
$$

Then $V_{\alpha, n}$ is represented by the fs $\log$ scheme over $S_{n}$

$$
\operatorname{Spec}\left(\mathbb{Z}\left[N_{\alpha}\right] \otimes_{\mathbb{Z}[\mathbb{N}]} O_{K} / m_{K}^{n}\right)
$$

endowed with the log structure associated to $N_{\alpha} \rightarrow \mathbb{Z}\left[N_{\alpha}\right] \otimes_{\mathbb{Z}[\mathbb{N}]} O_{K} / m_{K}^{n}$, where $\mathbb{N} \rightarrow O_{K}$ sends 1 to a fixed prime element $\pi$ of $K$ and the homomorphism $\mathbb{N} \rightarrow N_{\alpha}$ sends 1 to $(1,0)$. This is because for an object $U$ of $\left(\mathrm{fs} / S_{n}\right)$, we have

$$
\begin{aligned}
& \operatorname{Mor}_{S_{n}}\left(U, \operatorname{Spec}\left(\mathbb{Z}\left[N_{\alpha}\right] \otimes_{\mathbb{Z}[\mathbb{N}]} O_{K} / m_{K}^{n}\right)\right) \\
& \cong\left\{h \in \operatorname{Hom}\left(N_{\alpha}, M_{U}\right) \mid h(1,0)=\pi\right\} \cong V_{\alpha, n}(U),
\end{aligned}
$$

where the last isomorphism sends $h$ to $\varphi=h^{\mathrm{gp}}(0,1)$. Let $\mathbb{G}_{m, \log , n}^{(q, I)}$ be the restriction of $\mathbb{G}_{m, \log }^{(q, I)}$ to $\left(\mathrm{fs} / S_{n}\right)$. Then $\mathbb{G}_{m, \log , n}^{(q, I)}=\bigcup_{\alpha \in I} V_{\alpha, n}$. For $\alpha, \beta \in I$, $V_{\alpha, n} \cap V_{\beta, n}=\varnothing$ in $\mathbb{G}_{m, \log , n}^{(q, I)}$ unless $\alpha=\beta$ or $\alpha^{\prime}=\beta$ or $\beta^{\prime}=\alpha$, where $\alpha^{\prime}$ denotes the smallest element of $I$ satisfying $\alpha^{\prime}>\alpha$. For $\alpha \in I, V_{\alpha, n} \cap V_{\alpha^{\prime}, n}=$ $V_{\alpha, n}[1 / f]=V_{\alpha^{\prime}, n}[1 / g]$, where $f$ (resp. $g$ ) is any element $(a, b)$ of $N_{\alpha}$ (resp. $\left.N_{\alpha^{\prime}}\right)$ such that $a+\alpha^{\prime} e b=0, b \neq 0$ which we regard as an element of $\mathcal{O}\left(V_{\alpha, n}\right)$ (resp. $\mathcal{O}\left(V_{\alpha^{\prime}, n}\right)$ ), and hence $V_{\alpha, n} \cap V_{\alpha^{\prime}, n}$ is an open fs log subscheme of $V_{\alpha, n}$ and is also an open fs $\log$ subscheme of $V_{\alpha^{\prime}, n}$. Hence $\mathbb{G}_{m, \log , n}^{(q, I)}$ is representable and $\left(V_{\alpha, n}\right)_{\alpha \in I}$ is a covering of $\mathbb{G}_{m, \log , n}^{(q, I)}$ by open fs log subschemes. Since 
$q V_{\alpha, n}=V_{\alpha+1, n}$, the quotient sheaf $\mathbb{G}_{m, \log , n}^{(q, I)} / q^{\mathbb{Z}}$ is represented by the quotient of the fs $\log$ scheme $\mathbb{G}_{m, \log , n}^{(q, I)}$ over $S_{n}$ by the action of $q^{\mathbb{Z}}$. The formal scheme $\lim _{\longrightarrow n} \mathbb{G}_{m, \log , n}^{(q, I)} / q^{\mathbb{Z}}$ is projective and algebraizable over $O_{K}$. This is $\mathcal{E}_{q}^{(I)}$. It can be seen easily that with the log structure defined in 1.4.1, the restriction of $\mathcal{E}_{q}^{(I)}$ to (fs $\left./ S\right)^{\prime}$ coincides with $\mathbb{G}_{m, \log }^{(q, I)} / q^{\mathbb{Z}}$.

1.6. Now we give an explicit construction of $\mathcal{E}_{q}$ :

$$
\mathcal{E}_{q}=\left(\coprod_{(i, I)} \mathcal{E}_{q^{i}}^{(I)}\right) / \sim
$$

where $(i, I)$ ranges over all pairs with $i$ an integer $\geq 1$ and $I$ a non-empty subset of $\mathbb{Q}$ stable under the translation by $\mathbb{Z}$ such that $I / \mathbb{Z}$ is finite, and $\sim$ is the equivalence relation in the category of sheaves on $(\mathrm{fs} / S)$ generated by the following equivalences 1.6.1 and 1.6.2.

1.6.1. For any $(i, I)$ and $(j, J)$ such that $j \mid i$ and such that $(i / j) I \supset J$, any element of $\mathcal{E}_{q^{i}}^{(I)}(U)$ for any fs $\log$ scheme $U$ over $S$ is equivalent to its image in $\mathcal{E}_{q^{j}}^{(J)}(U)$ under the canonical morphism $\mathcal{E}_{q^{i}}^{(I)} \rightarrow \mathcal{E}_{q^{j}}^{(J)}$.

Here the morphism $\mathcal{E}_{q^{i}}^{(I)} \rightarrow \mathcal{E}_{q^{j}}^{(J)}$ is the one whose restriction to (fs $\left./ S\right)^{\prime}$ is the evident map of sheaves. Such a morphism uniquely exists since these models are proper over $S$.

1.6.2. For any $(i, I)$, if we denote $J=I+(1 / i)$, any element of $\mathcal{E}_{q^{i}}^{(I)}(U)$ for any fs $\log$ scheme $U$ over $S$ is equivalent to its image in $\mathcal{E}_{q^{i}}^{(J)}(U)$ under the morphism $\mathcal{E}_{q^{i}}^{(I)} \rightarrow \mathcal{E}_{q^{i}}^{(J)}$ whose pull back to (fs $\left./ S\right)^{\prime}$ is induced by $\mathbb{G}_{m, \log }^{(q)} / q^{i \mathbb{Z}} \rightarrow$ $\mathbb{G}_{m, \log }^{(q)} / q^{i \mathbb{Z}} ; \varphi \mapsto q \varphi$.

The following proposition will be proved in Section 5 .

Proposition 1.7. (1) The pull back of $\mathcal{E}_{q}$ to (fs/Spec $(K)$ ) coincides with $E_{q}$. The pull back of $\mathcal{E}_{q}$ to $(\mathrm{fs} / S)^{\prime}$ coincides with $\mathbb{G}_{m, \log }^{(q)} / q^{\mathbb{Z}}$.

(2) There exists a unique group law on $\mathcal{E}_{q}$ whose pull back to $(\mathrm{fs} / \operatorname{Spec}(K))$ coincides with the group law of $E_{q}$ and whose pull back to $(\mathrm{fs} / S)^{\prime}$ coincides with the group law of $\mathbb{G}_{m, \log }^{(q)} / q^{\mathbb{Z}}$.

(3) The canonical morphism $\mathcal{E}_{q}^{(I)} \rightarrow \mathcal{E}_{q}$ is injective. As a subsheaf of $\mathcal{E}_{q}$, the sheaf $\mathcal{E}_{q}^{(I)}$ coincides with the inverse image of $\mathbb{G}_{m, \log }^{(q, I)} /\left(\mathbb{G}_{m} q^{\mathbb{Z}}\right)$ under the canonical morphism $\mathcal{E}_{q} \rightarrow \mathbb{G}_{m, \log }^{(q)} /\left(\mathbb{G}_{m} q^{\mathbb{Z}}\right)$ on $(\mathrm{fs} / S)$. 
1.8. Question 2 on the best model is related to compactifications of moduli spaces of elliptic curves studied in Deligne-Rapoport [1]. Let $n \geq 1$ be an integer which is invertible in $O_{K}$, let $Y(n)$ be the fine moduli space over $\mathbb{Z}[1 / n]$ of elliptic curves with $n$-level structures, and let $X(n)$ be the canonical compactification of $Y(n)$ in [1]. Assume that the group of $n$ division points in $E_{q}(K)$ is isomorphic to $(\mathbb{Z} / n \mathbb{Z})^{2}$ and fix such an isomorphism. Then $E_{q}$ with this $n$-level structure defines a morphism $\operatorname{Spec}(K) \rightarrow$ $Y(n)$. Since $X(n)$ is proper, this morphism is extended uniquely to a commutative diagram

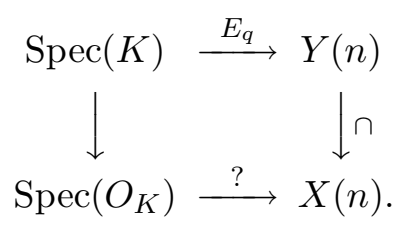

In [1], the space $X(n)$ is a moduli space of degenerating elliptic curves (called generalized elliptic curves) with $n$-level structures. That is, the lower horizontal arrow corresponds to a generalized elliptic curve ? over $O_{K}$ with $n$-level structure which is a candidate of the best model of $E_{q}$ from the viewpoint of compactifications of moduli spaces. This model, an answer to Question 2 , is $\mathcal{E}_{q}^{((1 / n) \mathbb{Z})}$ in our notation. Thus the answer depends on $n$.

We will take $\mathcal{E}_{q}$ instead of $\mathcal{E}_{q}^{((1 / n) \mathbb{Z})}$ at the place ? when we discuss compactifications of moduli spaces of abelian varieties in the sequel of this Part II. Advantages of $\mathcal{E}_{q}$ are that it is independent of $n$ and furthermore, since it has a group structure, the meaning of $n$-level structure becomes simple. We already discussed the analytic version in [6].

\section{§2. Log 1-motifs}

In this section, we define the notion log 1-motif, as a preparation to introduce the notion log abelian variety. The proofs of Proposition 2.5 and Remark 2.8.2 will be given in Section 6 .

Let $S$ be an fs log scheme and let (fs/S) be the category of fs log schemes over $S$. We regard (fs/S) as a site with the usual étale topology as in 1.2. We identify an fs log scheme $V$ over $S$ with the sheaf on (fs $/ S$ ) represented by $V$. For a point $v$ of $V$ and a sheaf $F$ on $(\mathrm{fs} / S)$, we denote by $F_{\bar{v}}$ the stalk at $v$ of the sheaf $\left.F\right|_{V_{\text {ét }}}$ obtained by restricting $F$ to the small étale site $V_{\text {ét }}$ of $V$. Note that this $F_{\bar{v}}$ often differs from the set $F(\bar{v})$ of $\bar{v}$-sections, where $\bar{v}$ denotes the Spec of the separable closure of the residue field of $v$, and is endowed with the inverse image of the $\log$ structure of $V$ (as is so 
already in the non-log case; say, in case where $F=\mathbb{G}_{a}$, we have $F_{\bar{v}}=\mathcal{O}_{V, \bar{v}}$ and $\left.F(\bar{v})=\Gamma\left(\bar{v}, \mathcal{O}_{\bar{v}}\right)\right)$.

2.1. Let $G$ be a commutative group scheme over the underlying scheme $S$ which is an extension of an abelian scheme $B$ by a torus $T$. We define the logarithmic enlargement $G_{\log }$ of $G$, which is a sheaf of abelian groups on (fs/S) containing $G$, as follows.

We regard $G$ as a sheaf of abelian groups on (fs $/ S$ ): For an fs log scheme $U$ over $S, G(U)$ is defined by forgetting the $\log$ structure of $U$. This sheaf is represented by $G$ which is endowed with the inverse image of the log structure of $S$.

Let $X:=\mathcal{H} \operatorname{om}\left(T, \mathbb{G}_{m}\right)$ be the character group of $T$ (here $\mathcal{H o m}$ denotes the hom sheaf), which is a locally constant sheaf of finitely generated free $\mathbb{Z}$-modules on $(\mathrm{fs} / S)$, and let $T_{\log }:=\mathcal{H} \operatorname{Hom}\left(X, \mathbb{G}_{m, \log }\right)$. Here the sheaves $\mathbb{G}_{m}$ and $\mathbb{G}_{m, \log }$ on $(\mathrm{fs} / S)$ are defined by

$$
\mathbb{G}_{m}(U)=\Gamma\left(U, \mathcal{O}_{U}^{\times}\right), \quad \mathbb{G}_{m, \log }(U)=\Gamma\left(U, M_{U}^{\mathrm{gp}}\right)
$$

for an fs $\log$ scheme $U$ over $S$. We have a canonical embedding $T \subset T_{\log }$.

We define $G_{\log }$ as the pushout of $T_{\log } \leftarrow T \rightarrow G$ in the category of sheaves of abelian groups on (fs $/ S)$. We have a commutative diagram with exact rows

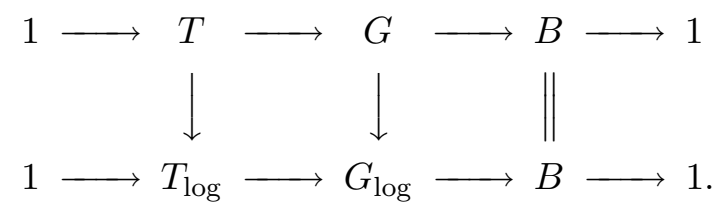

Definition 2.2. A $\log 1$-motif $M$ over $S$ consists of the following data:

(a) a commutative group sheaf $Y$ on $(\mathrm{fs} / S)$ which is, étale locally on $S$, isomorphic to the constant sheaf defined by a free $\mathbb{Z}$-module of finite rank;

(b) a commutative group scheme $G$ over (the underlying scheme of) $S$ which is an extension of an abelian scheme $B$ over $S$ by a torus $T$ over $S$;

(c) a homomorphism $u: Y \rightarrow G_{\log }$.

We denote by $M=\left[u: Y \rightarrow G_{\log }\right]$ a $\log 1$-motif over $S$ as a complex of sheaves on $S$ with $G_{\log }$ degree 0 .

Putting $X=\mathcal{H} o m\left(T, \mathbb{G}_{m}\right)$, we say $M$ is a log 1-motif of type $(X, Y)$. 
2.3. We define a canonical pairing $\langle\rangle:, X \times Y \rightarrow \mathbb{G}_{m, \log } / \mathbb{G}_{m}$ for a $\log 1$-motif $\left[u: Y \rightarrow G_{\log }\right]$, where $X=\mathcal{H} \operatorname{Hom}\left(T, \mathbb{G}_{m}\right)$. Since the left square in (2.1.1) is a pushout, we have isomorphisms $G_{\log } / G \cong T_{\log } / T \cong$ $\mathcal{H o m}\left(X, \mathbb{G}_{m, \log } / \mathbb{G}_{m}\right)$. Hence we have a natural pairing

$$
\langle,\rangle: X \times Y \longrightarrow X \times\left(G_{\log } / G\right) \longrightarrow \mathbb{G}_{m, \log } / \mathbb{G}_{m} .
$$

Definition 2.4. Let $M=\left[u: Y \rightarrow G_{\log }\right], M^{\prime}=\left[u^{\prime}: Y^{\prime} \rightarrow G_{\log }^{\prime}\right]$ be $\log$ 1-motifs over $S$. A morphism $h: M \rightarrow M^{\prime}$ is a homomorphism of complexes

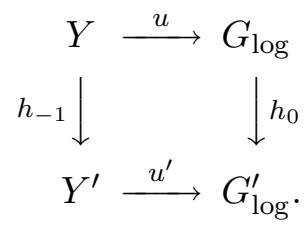

Proposition 2.5. Let $G$ and $G^{\prime}$ be commutative group schemes which are extensions of abelian schemes by tori. Then we have an isomorphism

$$
\operatorname{Hom}\left(G, G^{\prime}\right) \stackrel{\cong}{\longrightarrow} \operatorname{Hom}\left(G_{\log }, G_{\log }^{\prime}\right) .
$$

The proof will be given in Section 6 .

Corollary 2.6. $\mathcal{H o m}\left(T_{\log }, \mathbb{G}_{m, \log }\right)=X$.

2.7. Let $M:=\left[u: Y \rightarrow G_{\log }\right]$ be a $\log 1$-motif of type $(X, Y)$ over $S$. The dual $\log 1$-motif $M^{*}$ to $M$ is the $\log 1$-motif $\left[u^{*}: X \rightarrow G_{\log }^{*}\right]$ of type $(Y, X)$ defined as follows.

2.7.1. Let $B^{*}=\mathcal{E} x t\left(B, \mathbb{G}_{m}\right)$ be the dual abelian scheme of $B$, and $T^{*}:=\mathcal{H o m}\left(Y, \mathbb{G}_{m}\right)$ the torus with character group $Y$. We define $G^{*}$ as the sheafification of the presheaf

$$
U \longmapsto\left\{\text { pairs }(F, h) \text { of an extension } F \text { of } B \text { by } \mathbb{G}_{m} \text { over } U\right. \text { and }
$$
a homomorphism $h: Y \rightarrow F$ such that the composition $Y \stackrel{h}{\rightarrow} F \rightarrow B$ coincides with $\left.Y \stackrel{u}{\rightarrow} G_{\log } \stackrel{\text { pr }}{\rightarrow} B\right\} / \cong$.

Here pr: $G_{\log } \rightarrow B$ is the canonical projection. We have an exact sequence

$$
1 \longrightarrow T^{*} \longrightarrow G^{*} \longrightarrow B^{*} \longrightarrow 1
$$


where $G^{*} \rightarrow B^{*}$ sends the class of $(F, h)$ to that of $F$, and the map $T^{*} \rightarrow G^{*}$ sends $\left(t: Y \rightarrow \mathbb{G}_{m}\right) \in T^{*}$ to the class of $\left(\mathbb{G}_{m} \oplus B, t \oplus(\operatorname{pr} \circ u): Y \rightarrow \mathbb{G}_{m} \oplus B\right)$. Thus $G^{*}$ is representable. We can also write

$$
G^{*}=\mathcal{E} x t\left([Y \stackrel{\text { prou }}{\longrightarrow} B], \mathbb{G}_{m}\right),
$$

where $B$ (resp. $Y$ ) is in the degree 0 (resp. -1 ), and $\mathcal{E} x t$ is the $\operatorname{Ext}^{1}$-sheaf for objects in the derived category.

2.7.2. Then we have the sheaf of commutative groups $G_{\log }^{*}$ associated to $G^{*}$. We can also identify $G_{\log }^{*}$ with the sheafification of the presheaf

$U \longmapsto\left\{\right.$ pairs $(F, h)$ of an extension $F$ of $B$ by $\mathbb{G}_{m}$ over $U$ and a homomorphism $h: Y \rightarrow F_{\log }$ such that the composition $Y \stackrel{h}{\rightarrow} F_{\log } \rightarrow B_{\log }=B$ coincides with $\left.Y \stackrel{u}{\rightarrow} G_{\log } \stackrel{\text { pr }}{\rightarrow} B\right\} / \cong$.

2.7.3. Let $u^{*}: X \rightarrow G_{\log }^{*}$ be the homomorphism which sends $x \in X$ to the class of $(F, h)$, where $F$ is the extension obtained as

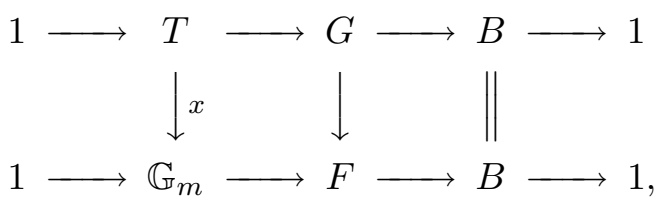

and $h$ is the composite homomorphism $Y \rightarrow G_{\log } \rightarrow F_{\log }$.

Definition 2.7.4. The dual $\log 1$-motif $M^{*}$ to $M=\left[u: Y \rightarrow G_{\log }\right]$ is the $\log 1$-motif $\left[u^{*}: X \rightarrow G_{\log }^{*}\right]$ of type $(Y, X)$ defined as above. Note that, by construction, we can easily show that $M^{* *}$ is canonically isomorphic to $M$.

Definition 2.8. Let $M=\left[u: Y \rightarrow G_{\log }\right]$ be a $\log 1$-motif over $S$. A polarization of $M$ is a homomorphism $h: M \rightarrow M^{*}=\left[u^{*}: X \rightarrow G_{\log }^{*}\right]$ satisfying the following conditions (a) $-(\mathrm{d})$ :

(a) The homomorphism $B \rightarrow B^{*}$ induced by $h_{0}: G_{\log } \rightarrow G_{\log }^{*}$ is a polarization on $B$;

(b) The homomorphism $h_{-1}: Y \rightarrow X$ is injective and has finite cokernel;

(c) $\left\langle h_{-1}(y), y\right\rangle_{\bar{s}} \in\left(M_{S, \bar{s}} / \mathcal{O}_{S, \bar{s}}^{\times}\right) \backslash\{1\}$ for $y \in Y_{\bar{s}} \backslash\{0\}$ and $s \in S$. Here $\langle\rangle:, X \times Y \rightarrow M_{S}^{\mathrm{gp}} / \mathcal{O}_{S}^{\times}$is the pairing in 2.3; 
(d) The homomorphism $T_{\log } \rightarrow T_{\log }^{*}$ induced by $h_{0}: G_{\log } \rightarrow G_{\log }^{*}$ coincides with the one induced by $h_{-1}: Y \rightarrow X$.

We say that $M$ is pointwise polarizable if the pullback of $M$ to $(\mathrm{fs} / \bar{s})$ admits a polarization for every $s \in S$.

EXAMPLE 2.8.1. We give an example of polarization. Let $X=Y=\mathbb{Z}$ and let $q$ be an element of $\Gamma\left(S, M_{S}\right)$ such that $\alpha(q)$ (the image of $q$ in $\left.\Gamma\left(S, \mathcal{O}_{S}\right)\right)$ is locally nilpotent. Then the identity map from the log 1-motif $M=\left[Y \stackrel{1 \mapsto q}{\longrightarrow} \mathbb{G}_{m, \log }\right]$ to its dual $\left[X \stackrel{1 \mapsto q}{\longrightarrow} \mathbb{G}_{m, \log }\right]$ is a polarization of $M$. In the situation in Section 1, for $q \in m_{K} \backslash\{0\}$, we have thus polarizations of the $\log 1$-motif $\left[\mathbb{Z} \stackrel{1 \mapsto q}{\longrightarrow} \mathbb{G}_{m, \log }\right]$ over $S=\operatorname{Spec}\left(O_{K} / m_{K}^{n}\right)$ for $n \geq 1$.

Remark 2.8.2. The condition (c) is equivalent to the following:

$\left(\mathrm{c}^{\prime}\right)$ For any étale scheme $U$ over the underlying scheme of $S$ and for any $y \in Y(U)$ such that $y \neq 0$ at each point of $U$, the local section $m:=$ $\left\langle h_{-1}(y), y\right\rangle$ belongs to $\left(M_{S} / \mathcal{O}_{S}^{\times}\right)(U)$, and for any local lift $\widetilde{m} \in M_{S}$ of $m$, $\alpha(\widetilde{m})$ is locally nilpotent.

See 6.2 for the proof of the equivalence.

Remark 2.8.3. The injectivity in the condition (b) in fact follows from the condition (c).

Remark 2.8.4. It is easy to see that the existence of a polarization implies that $Y \rightarrow G_{\log } / G$ is injective.

\section{$\S 3$. Log abelian varieties with constant degeneration}

In this section, we define the notion log abelian varieties with constant degeneration, and state a category equivalence between pointwise polarizable $\log 1$-motifs and log abelian varieties with constant degeneration. Since the proof of the result Theorem 3.4 is involved and long, we give it later in Sections 7-8.

In the situation of Section 1, the pull back of $\mathcal{E}_{q}$ to $\left(\mathrm{fs} / S_{n}\right)$ with $S_{n}=$ $\operatorname{Spec}\left(O_{K} / m_{K}^{n}\right)$ is a log abelian variety with constant degeneration over $S_{n}$, but $\mathcal{E}_{q}$ itself is not a log abelian variety with constant degeneration over $O_{K}$ though it is a $\log$ abelian variety over $O_{K}$ as is explained in Section 9.

Let $S$ be an fs log scheme. 
3.1. Let $X$ and $Y$ be locally constant sheaves of finitely generated $\mathbb{Z}$ modules on $(\mathrm{fs} / S)$, and let $\langle\rangle:, X \times Y \rightarrow\left(\mathbb{G}_{m, \log } / \mathbb{G}_{m}\right)_{S}$ be a $\mathbb{Z}$-bilinear form.

We define a subgroup sheaf $\mathcal{H o m}\left(X, \mathbb{G}_{m, \log } / \mathbb{G}_{m}\right)^{(Y)}$ of the sheaf $\mathcal{H o m}\left(X, \mathbb{G}_{m, \log } / \mathbb{G}_{m}\right)$ on $(\mathrm{fs} / S)$. For each fs $\log$ scheme $U$ over $S$, we set

$$
\begin{aligned}
& \operatorname{Hom}\left(X, \mathbb{G}_{m, \log } / \mathbb{G}_{m}\right)^{(Y)}(U) \\
& :=\left\{\varphi \in \mathcal{H} \text { om }\left(X, \mathbb{G}_{m, \log } / \mathbb{G}_{m}\right)(U) \mid \text { for every } u \in U \text { and } x \in X_{\bar{u}},\right. \\
& \left.\quad \text { there exist } y_{u, x}, y_{u, x}^{\prime} \in Y_{\bar{u}} \text { such that }\left\langle x, y_{u, x}\right\rangle_{\bar{u}}\left|\varphi(x)_{\bar{u}}\right|\left\langle x, y_{u, x}^{\prime}\right\rangle \bar{u}\right\} .
\end{aligned}
$$

Here, for $u \in U$ and $a, b \in\left(M_{U}^{\mathrm{gp}} / \mathcal{O}_{U}^{\times}\right)_{\bar{u}}, a \mid b$ means $a^{-1} b \in\left(M_{U} / \mathcal{O}_{U}^{\times}\right)_{\bar{u}}$. We can easily verify that $\mathcal{H o m}\left(X, \mathbb{G}_{m, \log } / \mathbb{G}_{m}\right)^{(Y)}$ is a subgroup sheaf of $\mathcal{H o m}\left(X, \mathbb{G}_{m, \log } / \mathbb{G}_{m}\right)$.

As is easily seen, the image of $Y \rightarrow \mathcal{H o m}\left(X, \mathbb{G}_{m, \log } / \mathbb{G}_{m}\right)$ is contained in $\mathcal{H o m}\left(X, \mathbb{G}_{m, \log } / \mathbb{G}_{m}\right)^{(Y)}$.

3.2. Let $\left[u: Y \rightarrow G_{\log }\right]$ be a $\log 1$-motif over $S$ of type $(X, Y)$.

Let $G_{\log }^{(Y)} \subset G_{\log }$ be the inverse image of $\mathcal{H o m}\left(X, \mathbb{G}_{m, \log } / \mathbb{G}_{m}\right)^{(Y)}$ under $G_{\log } \rightarrow G_{\log } / G \cong \mathcal{H o m}\left(X, \mathbb{G}_{m, \log } / \mathbb{G}_{m}\right)$.

Then the image of $Y \rightarrow G_{\log }$ is contained in $G_{\log }^{(Y)}$.

For example, for $q \in M_{S}(S)$ and for the $\log 1$-motif $\left[Y \stackrel{1 \mapsto q}{\longrightarrow} G_{\log }\right]$ with $Y=\mathbb{Z}$ and $G=\mathbb{G}_{m}$, we have $G_{\log }^{(Y)}=\mathbb{G}_{m, \log }^{(q)}$, where

$$
\begin{array}{r}
\mathbb{G}_{m, \log }^{(q)}(U)=\left\{\varphi \in \Gamma\left(U, M_{U}^{\mathrm{gp}}\right) \mid \text { locally on } U, \text { there exist } m, n \in \mathbb{Z}\right. \\
\text { such that } \left.q^{m}|\varphi| q^{n}\right\} .
\end{array}
$$

DEFINITION 3.3. (1) A log abelian variety with constant degeneration over $S$ is a sheaf of abelian groups on $(\mathrm{fs} / S)$ which is isomorphic to the quotient sheaf $G_{\log }^{(Y)} / Y$ for a pointwise polarizable log 1-motif $\left[u: Y \rightarrow G_{\log }\right]$ over $S$.

(2) A homomorphism of log abelian varieties with constant degeneration is a homomorphism as sheaves of abelian groups on (fs/S).

The proof of the following theorem will be given in Section 8 .

TheOREM 3.4. The functor $\left[u: Y \rightarrow G_{\log }\right] \mapsto G_{\log }^{(Y)} / Y$ induces an equivalence from the category of pointwise polarizable log 1-motifs over $S$ to that of log abelian varieties with constant degeneration over $S$. 


\section{$\S 4$. Log abelian varieties}

In this section, we define the notion log abelian varieties, and introduce some basic properties. Since the proofs of some properties are long, we will give the proofs for 4.3, 4.4, Proposition 4.5, Theorem 4.6, Proposition 4.7, and Proposition 4.8 in Section 9 and the proof for Theorem 4.10 in Sections 10-11.

Definition 4.1. A $\log$ abelian variety over an fs $\log$ scheme $S$ is a sheaf of abelian groups $A$ on (fs/S) satisfying the following conditions 4.1.1, 4.1.2, and 4.1.3.

4.1.1. For any $s \in S$, the pull back of $A$ to $(\mathrm{fs} / \bar{s})$ is a $\log$ abelian variety with constant degeneration.

4.1.2. The following holds étale locally on $S$. There are a semiabelian group scheme $G$ over (the underlying scheme of) $S$, finitely generated free $\mathbb{Z}$ modules $X$ and $Y$, a $\mathbb{Z}$-bilinear form $\langle\rangle:, X \times Y \rightarrow\left(\mathbb{G}_{m, \log } / \mathbb{G}_{m}\right)_{S}$ on $(\mathrm{fs} / S)$ (i.e. a $\mathbb{Z}$-bilinear form $\left.\langle\rangle:, X \times Y \rightarrow M_{S}^{\mathrm{gp}} / \mathcal{O}_{S}^{\times}\right)$satisfying the condition (i) below, and an exact sequence

$$
0 \longrightarrow G \longrightarrow A \longrightarrow \mathcal{H o m}\left(X, \mathbb{G}_{m, \log } / \mathbb{G}_{m}\right)^{(Y)} / \bar{Y} \longrightarrow 0 .
$$

Here $\bar{Y}$ denotes the image of $Y$ in $\mathcal{H o m}\left(X, \mathbb{G}_{m, \log } / \mathbb{G}_{m}\right)$. A semi-abelian group scheme over a scheme $V$ means, as usual, a smooth separated group scheme over $V$ each fiber of which is an extension of an abelian variety by a torus. Let $\bar{X}$ be the image of $X$ in $\mathcal{H o m}\left(Y, \mathbb{G}_{m, \log } / \mathbb{G}_{m}\right)$.

(i) For each $s \in S$, there is a homomorphism $\phi: \bar{Y}_{\bar{s}} \rightarrow \bar{X}_{\bar{s}}$ with finite cokernel such that $\langle\phi(y), z\rangle=\langle\phi(z), y\rangle$ in $\left(M_{S}^{\mathrm{gp}} / \mathcal{O}_{S}^{\times}\right)_{\bar{s}}$ for any $y, z \in \bar{Y}_{\bar{s}}$, and such that $\langle\phi(y), y\rangle \in\left(M_{S} / \mathcal{O}_{S}^{\times}\right)_{\bar{s}}$ in $\left(M_{S}^{\mathrm{gp}} / \mathcal{O}_{S}^{\times}\right)_{\bar{s}}$ for any $y \in \bar{Y}_{\bar{s}}$.

4.1.3. The diagonal morphism $A \rightarrow A \times A$ is represented by finite morphisms. (See 4.2.)

4.2. We give an explanation of 4.1.3.

Let $f: F_{1} \rightarrow F_{2}$ be a morphism of contravariant functors from $\left(\mathrm{fs}_{\mathrm{s}} / S\right)$ to the category of sets. We say $f$ is represented by finite morphisms if for any object $U$ of (fs $/ S$ ) and any $a: U \rightarrow F_{2}$ (i.e. $a \in F_{2}(U)$ ), the fiber product $U \times_{F_{2}} F_{1}$ defined by $a$ is represented by an fs $\log$ scheme over $U$ whose underlying scheme is finite over that of $U$.

We have 
(1) Let $f: U \rightarrow V$ be a morphism of fs $\log$ schemes over $S$. Then $f: \operatorname{Mor}_{S}(, U) \rightarrow \operatorname{Mor}_{S}(, V)$ is represented by finite morphisms if and only if the underlying morphism of schemes of $f$ is finite.

In fact, the only if part is clear. The if part follows from the fact that the underlying scheme of the fiber product in the category of fs log schemes is finite over the fiber product as schemes.

(2) Let $V$ be an fs $\log$ scheme over $S$. Then the diagonal $\operatorname{Mor}_{S}(, V) \rightarrow$ $\operatorname{Mor}_{S}(, V) \times \operatorname{Mor}_{S}(, V)$ is represented by finite morphisms if and only if the underlying scheme of $V$ is separated over that of $S$.

In fact, let $W$ be the fiber product of $V \rightarrow S \leftarrow V$ in the category of fs $\log$ schemes, and let $W^{\prime}$ be the fiber product as schemes. By (1), the diagonal $\operatorname{Mor}(, V) \rightarrow \operatorname{Mor}(, V) \times \operatorname{Mor}(, V)$ is represented by finite morphisms if and only if (the underlying morphism of schemes of) $V \rightarrow W$ is finite. If $V \rightarrow W$ is finite, $V \rightarrow W^{\prime}$ is finite since $W \rightarrow W^{\prime}$ is finite, and this shows that $V$ is separated over $S$. Conversely, if $V$ is separated over $S$, then $V \rightarrow W$ is a morphism of $W^{\prime}$-schemes which are finite over $W^{\prime}$, and hence is finite.

Since the equalizer of $f, g: U \rightarrow A$ for an fs $\log$ scheme $U$ over $S$ (i.e. the fiber product of $\operatorname{Mor}(, U) \stackrel{(f, g)}{\longrightarrow} A \times A \leftarrow A)$ is the equalizer of $f-g, 0: U \rightarrow$ $A$, the condition 4.1 .3 can be replaced by the following condition:

The 0 -section $S \rightarrow A$ is represented by finite morphisms.

4.3. In [6], we considered the notion "admissible pairing" $X \times Y \rightarrow$ $\left(\mathbb{G}_{m, \log } / \mathbb{G}_{m}\right)_{S}$. See the beginning of Section 7 for the definition of the admissibility. An equivalent definition of log abelian variety is given by replacing the part

$$
\begin{aligned}
& \text { [a } \left.\mathbb{Z} \text {-bilinear form }\langle,\rangle: X \times Y \rightarrow\left(\mathbb{G}_{m, \log } / \mathbb{G}_{m}\right)_{S}\right] \\
& \text { [satisfying the condition (i) below] }
\end{aligned}
$$

in 4.1 .2 by

$$
\text { [an admissible pairing } \left.\langle,\rangle: X \times Y \rightarrow\left(\mathbb{G}_{m, \log } / \mathbb{G}_{m}\right)_{S}\right] \text {. }
$$

The equivalence of the definitions is explained in Section 9. 
4.4. The semi-abelian scheme $G$ in 4.1 .2 in fact exists globally on $S$ and is uniquely determined by $A$, as is explained in Section 9 . We call $G$ the semi-abelian part of $A$.

We define the dimension of $A$ to be the relative dimension of $G$ over $S$, which is a locally constant function on $S$.

The sheaves of $\mathbb{Z}$-modules $\bar{X}=\operatorname{Image}\left(X \rightarrow \mathcal{H o m}\left(Y, \mathbb{G}_{m, \log } / \mathbb{G}_{m}\right)\right)$ and $\bar{Y}$, and the pairing $\bar{X} \times \bar{Y} \rightarrow \mathbb{G}_{m, \log } / \mathbb{G}_{m}$ are also determined by $A$ and exist globally on $S$, as is explained in Section 9.

The following results 4.5-4.8 are proved also in Section 9 .

Proposition 4.5. A log abelian variety with constant degeneration over $S$ is a $\log$ abelian variety over $S$.

TheOREm 4.6. (1) A log abelian variety over $S$ is a log abelian variety with constant degeneration if and only if the rank of the torus part of the fiber of the semi-abelian scheme $G$ is locally constant on $S$.

(2) If the sheaf $M_{S} / \mathcal{O}_{S}^{\times}$on the (small) étale site of $S$ is locally constant, then a log abelian variety over $S$ is a log abelian variety with constant degeneration over $S$.

Proposition 4.7. Let $K, O_{K}$ and $q$ be as in Section 1. Then the sheaf of abelian groups $\mathcal{E}_{q}$ (the q-Tate curve) in Section 1 is a log abelian variety over $\operatorname{Spec}\left(O_{K}\right)$.

We have log abelian varieties which are more global than the above example in 4.7 , as follows.

Proposition 4.8. Let $S$ be a 1-dimensional regular Noetherian excellent scheme, and let $G$ be a 1-dimensional semi-abelian scheme over $S$. Let $J$ be a finite set of closed points of $S$, let $S^{\prime}=S \backslash J$, and for $v \in J$, let $O_{v}$ be the completion of the local ring $\mathcal{O}_{S, v}$, and let $K_{v}$ be the field of fractions of $O_{v}$. Assume that $G \times_{S} S^{\prime}$ is an abelian scheme, and for each $v \in J$, $G \times{ }_{S} \operatorname{Spec}\left(K_{v}\right)$ is a $q_{v}$-Tate curve over $K_{v}$, where $q_{v}$ is a non-zero element of the maximal ideal $m_{v}$ of $O_{v}$. Endow $S$ with the log structure associated to the divisor $J$, and endow $\operatorname{Spec}\left(O_{v}\right)\left(r e s p . \operatorname{Spec}\left(K_{v}\right)\right)$ with the canonical (resp. trivial) log structure. 
(1) For any $f$ s log scheme $U$ over $S$, define the group $A(U)$ by the cartesian diagram

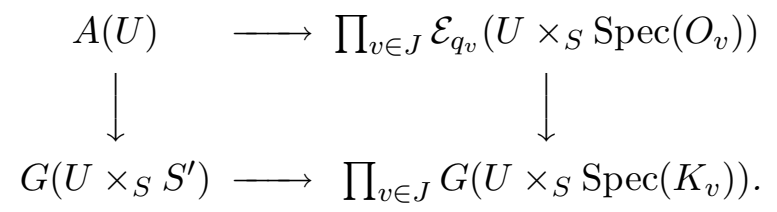

Here $\mathcal{E}_{q_{v}}$ is the $q_{v}$-Tate curve over $O_{v}$ in Proposition 4.7. Then $A$ is a (1-dimensional) log abelian variety over $S$.

(2) The pull back of $A$ to $S^{\prime}$ coincides with that of $G$. For each $v \in J$, the pull back of $A$ to $\left(\mathrm{fs} / O_{v}\right)$ coincides with $\mathcal{E}_{q_{v}}$.

(3) We have an exact sequence

$$
0 \longrightarrow G \longrightarrow A \longrightarrow \bigoplus_{v \in J} i_{v *}\left(\mathbb{G}_{m, \log }^{\left(q_{v}\right)} /\left(\mathbb{G}_{m} \cdot q_{v}^{\mathbb{Z}}\right)\right) \longrightarrow 0
$$

Here $i_{v *}\left(\mathbb{G}_{m, \log }^{\left(q_{v}\right)} /\left(\mathbb{G}_{m} \cdot q_{v}^{\mathbb{Z}}\right)\right)(U)=H^{0}\left(U \times_{S} v, \mathbb{G}_{m, \log }^{\left(q_{v}\right)} /\left(\mathbb{G}_{m} \cdot q_{v}^{\mathbb{Z}}\right)\right)$ for an $f_{s} \log$ scheme $U$ over $S$.

4.9. We give the relation with the theory of analytic log abelian varieties in $[6]$.

For an fs log scheme $S$ over $\mathbb{C}$ whose underlying scheme is locally of finite type over $\mathbb{C}$, let $S^{\text {an }}$ be the associated fs $\log$ analytic space. Let (fsan $/ S^{\text {an }}$ ) be the category of fs log analytic spaces over $S^{\text {an }}$. We regard (fsan $/ S^{\text {an }}$ ) as a site with respect to the usual topology. We have a morphism of sites (fsan $\left./ S^{\text {an }}\right) \rightarrow\left(\mathrm{fs}_{\mathrm{S}} / S\right)$ corresponding to the functor $(\mathrm{fs} / S) \rightarrow\left(\mathrm{fsan} / S^{\mathrm{an}}\right) ; U \mapsto U^{\mathrm{an}}$. For a sheaf $F$ on $(\mathrm{fs} / S)$, let $F^{\text {an }}$ be the pull back of $F$ on (fsan $\left./ S^{\text {an }}\right)$ by this morphism of sites. Then for an fs log scheme $U$ over $S$, we have

$$
\operatorname{Mor}_{S}(, U)^{\text {an }}=\operatorname{Mor}_{S \text { an }}\left(, U^{\text {an }}\right) .
$$

(Note that this last equality does not hold for the morphism of small sites $S^{\text {an }} \rightarrow S_{\text {ét }}$ or $\left.S^{\text {an }} \rightarrow S_{\text {Zar }}.\right)$

THEOREM 4.10. Let $S$ be an $f$ s log scheme over $\mathbb{C}$ whose underlying scheme is locally of finite type over $\mathbb{C}$, and let $A$ be a log abelian variety over $S$. Then $A^{\text {an }}$ is a log abelian variety over $S^{\text {an }}$ in the sense of [6] 1.3.5. (See 4.11 below.) 
This last theorem will be proved in Section 11. There we show how the condition 4.1.3 in the definition of log abelian variety is essential to have this theorem.

4.11. For readers' convenience, we review here the definition of analytic $\log$ abelian variety. See [6] for details.

Let $S$ be an fs $\log$ analytic space. In this paragraph, a pairing into $\mathbb{G}_{m, \log , S}$ means a triple $(X, Y,\langle\rangle$,$) , where X$ and $Y$ are finitely generated free abelian groups, and $\langle$,$\rangle is a \mathbb{Z}$-bilinear form $X \times Y \rightarrow \mathbb{G}_{m, \log , S}$.

4.11.1. For a pairing $(X, Y,\langle\rangle$,$) into \mathbb{G}_{m, \log , S}$, a subgroup sheaf $\mathcal{H o m}\left(X, \mathbb{G}_{m, \log }\right)^{(Y)}$ of $\mathcal{H o m}\left(X, \mathbb{G}_{m, \log }\right)$ on $(\mathrm{fsan} / S)$ is defined in the same way as in 3.1. We call the cokernel of $Y \rightarrow \mathcal{H o m}\left(X, \mathbb{G}_{m, \log }\right)^{(Y)}$ the quotient associated to this pairing.

4.11.2. A pairing $(X, Y,\langle\rangle$,$) into \mathbb{G}_{m, \log , S}$ is said to be non-degenerate if for any point $s$ of $S$, the following (i) and (ii) are satisfied.

(i) The induced $\mathbb{Z}$-bilinear form $X \times Y \rightarrow \mathcal{S}:=M_{S, s}^{\mathrm{gp}} / \mathcal{O}_{S, s}^{\times}$is admissible in the sense of 7.1 .

(ii) Let $\sigma$ be the face $\{1\}$ of $\mathcal{S}$. Then the induced $\mathbb{R}$-bilinear form $\mathbb{R} \otimes X_{\sigma} \times \mathbb{R} \otimes Y_{\sigma} \rightarrow \mathbb{R} ;(x, y) \mapsto \log (|\langle x, y\rangle(s)|)$ is non-degenerate. (See 7.1 for $X_{\sigma}$ and $Y_{\sigma}$.)

4.11.3. A pairing $(X, Y,\langle\rangle$,$) into \mathbb{G}_{m, \log , S}$ is said to be polarizable if there is a homomorphism $p: Y \rightarrow X$ satisfying the following (i)-(iii).

(i) $p$ is injective and the cokernel of $p$ is finite.

(ii) $\langle p(y), z\rangle=\langle p(z), y\rangle$ for any $y, z \in Y$.

(iii) For any $y \in Y,\langle p(y), y\rangle \in M_{S}$ in $M_{S}^{\mathrm{gp}}$. For any $y \in Y \backslash\{0\}$, the map $\alpha: M_{S} \rightarrow \mathcal{O}_{S}$ sends $\langle p(y), y\rangle$ to a function on $S$ whose values are always of absolute value $<1$.

It is shown in [6] 1.2.8 that any polarizable pairing into $\mathbb{G}_{m, \log , S}$ is non-degenerate in the sense of 4.11.2.

4.11.4. A sheaf $A$ of abelian groups on ( $\mathrm{fsan} / S$ ) is said to be a log complex torus if, locally on $S$, there exists a non-degenerate pairing $(X, Y,\langle\rangle$, into $\mathbb{G}_{m, \log , S}$ such that $A$ is isomorphic to the quotient associated to it.

4.11.5. A log complex torus is called polarizable if there exists a polarizable pairing $(X, Y,\langle\rangle$,$) into \mathbb{G}_{m, \log , S}$ such that $A$ is isomorphic to the quotient associated to it. 
4.11.6. A log complex torus is said to be a log abelian variety if, for any $s \in S$, the pull back of it to (fsan/s) is polarizable. Here $s$ is endowed with the inverse image of the $\log$ structure of $S$.

\section{§5. Proofs of Section 1}

Let $K, O_{K}, m_{K}, S=\operatorname{Spec}\left(O_{K}\right)$, and $q \in m_{K} \backslash\{0\}$ be as in Section 1. In this section, we prove Proposition 1.7.

5.1. We prove (1) of Proposition 1.7. It is easy to deduce that the pull back of $\mathcal{E}_{q}$ to $(\mathrm{fs} / \operatorname{Spec}(K))$ is $E_{q}$ from the fact that the pull back of $\mathcal{E}_{q^{i}}^{(I)}$ to (fs/ $\operatorname{Spec}(K))$ is $E_{q^{i}}$ and from the fact that $E_{q}$ is the quotient of $E_{q^{i}}$ by the action of $q^{\mathbb{Z}} / q^{i \mathbb{Z}}$ as a sheaf for the étale topology.

We prove that the pull back of $\mathcal{E}_{q}$ to $(\mathrm{fs} / S)^{\prime}$ is $\mathbb{G}_{m, \log }^{(q)} / q^{\mathbb{Z}}$. We have evident morphisms on (fs $/ S)^{\prime}$ from the pull backs of the $\mathcal{E}_{q^{i}}^{(I)} \mathrm{s}$ to $\mathbb{G}_{m, \log }^{(q)} / q^{\mathbb{Z}}$ and evidently the equivalence relations are killed in $\mathbb{G}_{m, \log }^{(q)} / q^{\mathbb{Z}}$. Hence we have a morphism on $(\mathrm{fs} / S)^{\prime}$ from the pull back of $\mathcal{E}_{q}$ to $\mathbb{G}_{m, \log }^{(q)} / q^{\mathbb{Z}}$. Next we will give a morphism on $(\mathrm{fs} / S)^{\prime}$ from $\mathbb{G}_{m, \log }^{(q)}$ to the pull back of $\mathcal{E}_{q}$. Let $\varphi$ be a local section of $\mathbb{G}_{m, \log }^{(q)}$. Locally, take a positive integer $m$ such that $q^{-m}|\varphi| q^{m}$. Consider the image of $\varphi$ in the pull back of $\mathcal{E}_{q^{2 m}}^{(1 / 2+\mathbb{Z})}$ to (fs/S)', and then the image of it in the pull back of $\mathcal{E}_{q}$ to $(\mathrm{fs} / S)^{\prime}$. This gives a welldefined morphism from $\mathbb{G}_{m, \log }^{(q)}$ to the pull back of $\mathcal{E}_{q}$, which factors through the quotient by $q^{\mathbb{Z}}$ and induces the converse of the above morphism on $(\text { fs } / S)^{\prime}$ from the pull back of $\mathcal{E}_{q}$ to $\mathbb{G}_{m, \log }^{(q)} / q^{\mathbb{Z}}$.

Lemma 5.2. The map $\mathcal{E}_{q}^{(I)} \rightarrow \mathcal{E}_{q}$ is injective.

Proof. By a limit argument, it is sufficient to prove that the map $\mathcal{E}_{q}^{(I)}(U) \rightarrow \mathcal{E}_{q}(U)$ is injective for any fs log scheme $U$ over $S$ whose underlying scheme is the Spec of a Noetherian ring $R$. Since $R \rightarrow\left(R \otimes_{O_{K}}\right.$ $K) \times \lim _{n}\left(R / m_{K}^{n} R\right)$ is faithfully flat and since $\mathcal{E}_{q}^{(I)}\left(\varliminf_{\varliminf_{n}} R / m_{K}^{n} R\right) \rightarrow$ $\lim _{n} \mathcal{E}_{q}^{(\overleftarrow{I)}}\left(R / m_{K}^{n} R\right)$ is bijective, the $\operatorname{map} \mathcal{E}_{q}^{(I)}(R) \rightarrow{\overleftarrow{\mathcal{E}_{q}^{(I)}}}^{n}\left(R \otimes_{O_{K}} K\right) \times$ $\varliminf_{n} \mathcal{E}_{q}^{(I)}\left(R / m_{K}^{n} R\right)$ is injective. Since $\mathcal{E}_{q}^{(I)}\left(R \otimes_{O_{K}} K\right)=E_{q}\left(R \otimes_{O_{K}} K\right)$, we are reduced to the injectivity of $\mathcal{E}_{q}^{(I)}\left(R / m_{K}^{n} R\right) \rightarrow \mathcal{E}_{q}\left(R / m_{K}^{n} R\right)$ which is clear from the descriptions of the restrictions of $\mathcal{E}_{q}^{(I)}$ and $\mathcal{E}_{q}$ to $(\mathrm{fs} / S)^{\prime}$. (For $\mathcal{E}_{q}$, the description was proved in 5.1.) 
5.3. We define a surjective morphism $\mathcal{E}_{q} \rightarrow \mathbb{G}_{m, \log }^{(q)} /\left(\mathbb{G}_{m} q^{\mathbb{Z}}\right)$ as follows. For an fs $\log$ scheme $U$ over $S$, this is given as

$$
\begin{array}{r}
\mathcal{E}_{q}(U) \longrightarrow \mathcal{E}_{q}\left(U \otimes_{O_{K}} O_{K} / m_{K}\right) \longrightarrow\left(\mathbb{G}_{m, \log }^{(q)} /\left(\mathbb{G}_{m} q^{\mathbb{Z}}\right)\right)\left(U \otimes_{O_{K}} O_{K} / m_{K}\right) \\
\cong\left(\mathbb{G}_{m, \log }^{(q)} /\left(\mathbb{G}_{m} q^{\mathbb{Z}}\right)\right)(U),
\end{array}
$$

where the second arrow is given by identifying the pull back of $\mathcal{E}_{q}$ to $(\mathrm{fs} / S)^{\prime}$ with $\mathbb{G}_{m, \log }^{(q)} / q^{\mathbb{Z}}$.

It is easy to see that $\mathcal{E}_{q}^{(I)}$ coincides with the inverse image of $\mathbb{G}_{m, \log }^{(q, I)} /\left(\mathbb{G}_{m} q^{\mathbb{Z}}\right)$ in $\mathcal{E}_{q}$. This completes the proof of $1.7(3)$.

5.4. We consider the semi-abelian scheme $G$ over $O_{K}$ which extends $E_{q}$ (see [1], [2] for example). It is the open set of the proper model $\mathcal{E}_{q}^{(\mathbb{Z})}$, which is the union of the general fiber of $\mathcal{E}_{q}^{(\mathbb{Z})}$ and the smooth part of the special fiber of $\mathcal{E}_{q}^{(\mathbb{Z})}$. We endow $G$ with the inverse image of the $\log$ structure of $S$ (then $G(U)$ for an fs $\log$ scheme $U$ over $S$ coincides with the usual $G(U)$ defined by forgetting the $\log$ structure of $U$ ). As a sheaf on (fs $/ S$ ), $G$ coincides with the inverse image in $\mathcal{E}_{q}^{(\mathbb{Z})}$ of the unit section of $\mathbb{G}_{m, \log }^{(q)} /\left(\mathbb{G}_{m} q^{\mathbb{Z}}\right)$. In particular, the pull back of $G$ to $(\mathrm{fs} / S)^{\prime}$ coincides with $\mathbb{G}_{m}$.

There is a unique action of $G$ on $\mathcal{E}_{q}^{(\mathbb{Z})}$ whose pull back to (fs/ $\left.\operatorname{Spec}(K)\right)$ coincides with the group law $E_{q} \times E_{q} \rightarrow E_{q}$, and whose pull back to (fs $\left./ S\right)^{\prime}$ is induced from the evident action of $\mathbb{G}_{m}$ on $\mathbb{G}_{m, \log }^{(q)} / q^{\mathbb{Z}}$ (cf. [1]).

5.5. Consider the proper model $\left(\mathcal{E}_{q}^{(\mathbb{Z})} \times \mathcal{E}_{q}^{(\mathbb{Z})}\right)^{\prime}$ of $E_{q} \times E_{q}$ whose pull back to $(\mathrm{fs} / S)^{\prime}$ is

$$
\begin{aligned}
& \left\{\left(\varphi_{1}, \varphi_{2}\right) \in \mathbb{G}_{m, \log } \times \mathbb{G}_{m, \log } \mid \text { locally there exist } a, b, c \in \mathbb{Z}\right. \text { such that } \\
& \left.\qquad q^{a}\left|\varphi_{1}\right| q^{a+1}, q^{b}\left|\varphi_{2}\right| q^{b+1}, q^{c}\left|\varphi_{1}^{-1} \varphi_{2}\right| q^{c+1}\right\} /\left(q^{\mathbb{Z}} \times q^{\mathbb{Z}}\right) .
\end{aligned}
$$

The existence of this proper model is shown as in the same way as the existence of $\mathcal{E}_{q}^{(I)}$ in 1.5. We have a canonical log étale morphism of proper models $\left(\mathcal{E}_{q}^{(\mathbb{Z})} \times \mathcal{E}_{q}^{(\mathbb{Z})}\right)^{\prime} \rightarrow \mathcal{E}_{q}^{(\mathbb{Z})} \times \mathcal{E}_{q}^{(\mathbb{Z})}$, and a morphism $\nu:\left(\mathcal{E}_{q}^{(\mathbb{Z})} \times \mathcal{E}_{q}^{(\mathbb{Z})}\right)^{\prime} \rightarrow \mathcal{E}_{q}^{(\mathbb{Z})}$ whose pull back to (fs $/ S)^{\prime}$ is given by $\left(\varphi_{1}, \varphi_{2}\right) \mapsto \varphi_{1}^{-1} \varphi_{2}$.

The open immersion $G \times \mathcal{E}_{q}^{(\mathbb{Z})} \rightarrow \mathcal{E}_{q}^{(\mathbb{Z})} \times \mathcal{E}_{q}^{(\mathbb{Z})}$ factors uniquely through an open immersion $G \times \mathcal{E}_{q}^{(\mathbb{Z})} \rightarrow\left(\mathcal{E}_{q}^{(\mathbb{Z})} \times \mathcal{E}_{q}^{(\mathbb{Z})}\right)^{\prime}$, and the action of $G$ on $\mathcal{E}_{q}^{(\mathbb{Z})}$ coincides with the composition $G \times \mathcal{E}_{q}^{(\mathbb{Z})} \rightarrow G \times \mathcal{E}_{q}^{(\mathbb{Z})} \rightarrow\left(\mathcal{E}_{q}^{(\mathbb{Z})} \times \mathcal{E}_{q}^{(\mathbb{Z})}\right)^{\prime} \stackrel{\nu}{\rightarrow} \mathcal{E}_{q}^{(\mathbb{Z})}$, where the first arrow is $(g, f) \mapsto\left(g^{-1}, f\right)$, the second arrow is the above open immersion, and the last arrow is $\nu$. 
Lemma 5.6. Let $U$ be an $f$ s $\log$ scheme over $S$. Let $f, g \in \mathcal{E}_{q}(U)$. Then étale locally on $U$, there exist an integer $m \geq 1$ and sections $\widetilde{f}, \widetilde{g}$ of $\mathcal{E}_{q^{m}}^{(\mathbb{Z})}$ such that $f$ comes from $\tilde{f}, g$ comes from $\widetilde{g}$, and $(\tilde{f}, \widetilde{g})$ belongs to $\left(\mathcal{E}_{q^{m}}^{(\mathbb{Z})} \times \mathcal{E}_{q^{m}}^{(\mathbb{Z})}\right)^{\prime}$. Here $\left(\mathcal{E}_{q^{m}}^{(\mathbb{Z})} \times \mathcal{E}_{q^{m}}^{(\mathbb{Z})}\right)^{\prime}$ is defined as in 5.5 replacing $q$ there by $q^{m}$.

Proof. We may assume that $f$ comes from $\mathcal{E}_{q^{i}}^{(I)}$ and $g$ comes from $\mathcal{E}_{q^{j}}^{(J)}$ for some $(i, I),(j, J)$. For $\alpha \in I$, let $\mathcal{E}_{q^{i}}^{(I, \alpha)}$ be the open fs log subscheme of $\mathcal{E}_{q^{i}}^{(I)}$ characterized by the property that for any fs log scheme $U$ over $S$, $\mathcal{E}_{q^{i}}^{(I, \alpha)}(U)$ consists of all elements of $\mathcal{E}_{q^{i}}^{(I)}(U)$ whose image in $\mathcal{E}_{q^{i}}^{(I)}\left(U \otimes_{O_{K}}\right.$ $\left.O_{K} / m_{K}\right)$ belongs to the image of $\left\{\varphi \in \mathbb{G}_{m, \log }\left|q^{k i \alpha}\right| \varphi^{k} \mid q^{k i \alpha^{\prime}}\right.$ for some $k \geq$ 1 such that $\left.k \alpha, k \alpha^{\prime} \in \mathbb{Z}\right\}$. Then $\mathcal{E}_{q^{i}}^{(I)}$ is covered by $\mathcal{E}_{q^{i}}^{(I, \alpha)}$ for $\alpha \in I$. Hence we may assume that $f$ comes from $\mathcal{E}_{q^{i}}^{(I, \alpha)}(U)$ and $g$ comes from $\mathcal{E}_{q^{j}}^{(J, \beta)}(U)$ for some $\alpha \in I, \beta \in J$. Furthermore we may assume $\alpha \geq 0$ and $i \alpha^{\prime} \leq j \beta$ (use the equivalence 1.6.2). Take a common multiple $m \geq 1$ of $i$ and $j$ such that $j \beta^{\prime} \leq m$. Then, since $0 \leq i \alpha, i \alpha^{\prime} \leq m, 0 \leq j \beta, j \beta^{\prime} \leq m$, and $0 \leq-i \alpha^{\prime}+j \beta,-i \alpha+j \beta^{\prime} \leq m$, étale locally, both $f$ and $g$ come from $\widetilde{f}$ and $\widetilde{g}$ in $\mathcal{E}_{q^{m}}^{(\mathbb{Z})}$ such that $(\widetilde{f}, \widetilde{g})$ belongs to $\left(\mathcal{E}_{q^{m}}^{(\mathbb{Z})} \times \mathcal{E}_{q^{m}}^{(\mathbb{Z})}\right)^{\prime}$.

Lemma 5.7. Let Spec $R$ be an fs log scheme over $S$ with $R$ a Noetherian ring. Then $\mathcal{E}_{q}(R) \rightarrow \mathcal{E}_{q}\left(R \otimes_{O_{K}} K\right) \times \lim _{n_{n}} \mathcal{E}_{q}\left(R / m_{K}^{n} R\right)$ is injective.

Proof. Let $f, g \in \mathcal{E}_{q}(R)$ and assume that the images of $f$ and $g$ in $\mathcal{E}_{q}\left(R \otimes_{O_{K}} K\right) \times \lim _{n} \mathcal{E}_{q}\left(R / m_{K}^{n} R\right)$ coincide. We prove $f=g$. By Lemma 5.6, étale locally on $\operatorname{Spec} R$, there exists $m \geq 1$ such that $f, g$ come from $\widetilde{f}, \widetilde{g} \in \mathcal{E}_{q^{m}}^{(\mathbb{Z})}$ and such that $(\widetilde{f}, \widetilde{g}) \in\left(\mathcal{E}_{q^{m}}^{(\mathbb{Z})} \times \mathcal{E}_{q^{m}}^{(\mathbb{Z})}\right)^{\prime}$. Since the images of $f, g$ in $\mathbb{G}_{m, \log }^{(q)} /\left(\mathbb{G}_{m} q^{\mathbb{Z}}\right)$ coincide, there exists $i \in \mathbb{Z}$ such that $\tilde{f}$ belongs to $\mathcal{E}_{q^{m}}^{((-i / m)+\mathbb{Z})}$ and the images of $q^{i} \widetilde{f}$ and $\widetilde{g}$ in $\mathbb{G}_{m, \log }^{(q)} /\left(\mathbb{G}_{m} q^{m \mathbb{Z}}\right)$ coincide. Let $G^{\prime}$ be the semi-abelian scheme over $O_{K}$ corresponding to $E_{q^{m}}$. Then $a:=\nu\left(q^{i} \widetilde{f}, \widetilde{g}\right)$ belongs to $G^{\prime}$, that is, $a \in G^{\prime}(R)$. Let $G$ be the semi-abelian scheme over $O_{K}$ which extends $E_{q}$. The image of $a$ in $G\left(R \otimes_{O_{K}} K\right)$ and the image of $a$ in $\varliminf_{n} G\left(R / m_{K}^{n} R\right)$ vanish. Since $R \rightarrow R \otimes_{O_{K}} K \times \lim _{n} R / m_{K}^{n} R$ is faithfully flat and since $G\left(\lim _{n} R / m_{K}^{n} R\right)=\varliminf_{n} G\left(R / m_{K}^{n} R\right)$ is bijective,

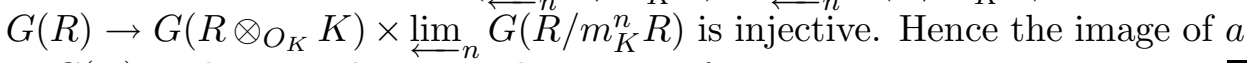
in $G(R)$ is the unit element. This proves $f=g$.

LEMma 5.8. In the situation of Lemma $5.7, \mathcal{E}_{q}(R)$ is a subgroup of $\mathcal{E}_{q}\left(R \otimes_{O_{K}} K\right) \times \lim _{n} \mathcal{E}_{q}\left(R / m_{K}^{n} R\right)$. 
Proof. Let $f, g \in \mathcal{E}_{q}(R)$. By Lemma 5.6, we may assume that $f, g$ come for some $m \geq 1$ from $\widetilde{f}, \widetilde{g} \in \mathcal{E}_{q^{m}}^{(\mathbb{Z})}$ such that $(\widetilde{f}, \widetilde{g})$ belongs to $\left(\mathcal{E}_{q^{m}}^{(\mathbb{Z})} \times \mathcal{E}_{q^{m}}^{(\mathbb{Z})}\right)^{\prime}$. Hence the product of $f^{-1}$ and $g$ takes values in $\mathcal{E}_{q^{m}}^{(\mathbb{Z})}$, and hence in the subset $\mathcal{E}_{q}(R)$ of $\mathcal{E}_{q}\left(R \otimes_{O_{K}} K\right) \times \lim _{n} \mathcal{E}_{q}\left(R / m_{K}^{n} R\right)$.

5.9. We prove the unique existence of the group structure of $\mathcal{E}_{q}$ stated in Section 1. The uniqueness follows from the injectivity in Lemma 5.7 by limit argument (which reduces the problem to Noetherian rings). Also we have the functorial group structure on $\mathcal{E}_{q}(R)$ by Lemma 5.8 and hence a group structure on $\mathcal{E}_{q}$ by the limit argument.

\section{$\S 6 . \quad$ Proofs of Section 2}

6.1. Proof of Proposition 2.5. To prove Proposition 2.5, we first show the following lemma:

Lemma 6.1.1. Let $H$ be a commutative group scheme over the underlying scheme of $S$ with connected fibers. Then $\operatorname{Hom}\left(H, \mathbb{G}_{m, \log } / \mathbb{G}_{m}\right)=0$.

Proof. Let $\varphi \in \operatorname{Hom}\left(H, \mathbb{G}_{m, \log } / \mathbb{G}_{m}\right)$, and $U$ an object of $\left(\mathrm{fs}_{\mathrm{s}} / S\right)$. We show that the map $H(U) \rightarrow\left(\mathbb{G}_{m, \log } / \mathbb{G}_{m}\right)(U)$ induced by $\varphi$ is trivial. For $u \in U$, we denote the Spec of the residue field of $u$ endowed with the inverse image of the log structure of $U$, simply by $u$. Since $\left(\mathbb{G}_{m, \log } / \mathbb{G}_{m}\right)(U) \rightarrow$ $\prod_{u \in U}\left(\mathbb{G}_{m, \log } / \mathbb{G}_{m}\right)(u)$ is injective, we are reduced to the case that (the underlying scheme of) $U$ is the Spec of a field. Consider the restriction of $\varphi$ to the full subcategory $\mathcal{C}$ of $(\mathrm{fs} / S) / U$ consisting of all objects whose $\log$ structures are the inverse images of the $\log$ structure of $U$. By assuming $U$ is the Spec of a field, the restriction of $\mathbb{G}_{m, \log } / \mathbb{G}_{m}$ to $\mathcal{C}$ is a locally constant sheaf, and hence is represented by an étale group scheme over $U$. Since a homomorphism of group schemes from $H \times{ }_{S} U$ to an étale group scheme over $U$ is trivial, the restriction of $\varphi$ to $\mathcal{C}$ is trivial. Hence the map $H(U) \rightarrow\left(\mathbb{G}_{m, \log } / \mathbb{G}_{m}\right)(U)$ induced by $\varphi$ is trivial.

Let $G, G^{\prime}$ be as in 2.5 .

Corollary 6.1.2. $\operatorname{Hom}\left(G, G_{\log }^{\prime} / G^{\prime}\right)=0$.

Proof. This follows from Lemma 6.1 .1 because $G_{\log }^{\prime} / G^{\prime}$ is étale locally isomorphic to a finite product of copies of $\mathbb{G}_{m, \log } / \mathbb{G}_{m}$. 

$G^{\prime}$

Lemma 6.1.3. Let $\varphi: G_{\log } \rightarrow G_{\log }^{\prime}$ be a homomorphism. Then $\varphi(G) \subset$

Proof. This follows from Corollary 6.1.2.

6.1.4. Now let $\varphi \in \operatorname{Hom}\left(G_{\log }, G_{\log }^{\prime}\right)$. By Lemma 6.1.3, the map $\varphi$ induces a homomorphism $\psi: G \rightarrow G^{\prime}$. Let $\psi_{\log }: G_{\log } \rightarrow G_{\log }^{\prime}$ be the homomorphism induced by $\psi$. We prove $\varphi=\psi_{\log }$. Since $\varphi-\psi_{\log }$ kills $G$, we have only to show $\operatorname{Hom}\left(G_{\log } / G, G_{\log }^{\prime}\right)=0$. Since $G_{\log } / G$ is étale locally isomorphic to a finite product of copies of $\mathbb{G}_{m, \log } / \mathbb{G}_{m}$, by replacing $G_{\log } / G$ by $\mathbb{G}_{m, \log } / \mathbb{G}_{m}$ and then $G^{\prime}$ by $G$, it is enough to prove the following lemma:

Lemma 6.1.5. $\operatorname{Hom}\left(\mathbb{G}_{m, \log } / \mathbb{G}_{m}, G_{\log }\right)=0$.

Proof. Let $W=\operatorname{Spec}\left(\mathcal{O}_{S}[t]\right)$ endowed with the log structure associated to $M_{S} \times \mathbb{N} \rightarrow \mathcal{O}_{S}[t]$ which sends $1 \in \mathbb{N}$ to $t$. Let $U$ be an object of $(\mathrm{fs} / S)$. Then, any section of $\mathbb{G}_{m, \log } / \mathbb{G}_{m}$ on $U$ is obtained, étale locally on $U$, as $a^{*}(t) b^{*}(t)^{-1} \bmod \mathbb{G}_{m}$, where $a, b: U \rightarrow W$. Hence it is sufficient to prove that any homomorphism $\varphi: \mathbb{G}_{m, \log } / \mathbb{G}_{m} \rightarrow G_{\log }$ sends $\left(t \bmod \mathbb{G}_{m}\right) \in\left(\mathbb{G}_{m, \log } / \mathbb{G}_{m}\right)(W)$ to $0 \in G_{\log }(W)$. Since $\left(t \bmod \mathbb{G}_{m}\right)$ vanishes on $W^{\prime}=\operatorname{Spec}\left(\mathcal{O}_{S}\left[t^{ \pm 1}\right]\right)$, the section $\varphi\left(t \bmod \mathbb{G}_{m}\right) \in G_{\log }(W)$ vanishes on $G_{\log }\left(W^{\prime}\right)$. Since $B(W) \rightarrow B\left(W^{\prime}\right)$ and $\mathbb{G}_{m, \log }(W) \rightarrow \mathbb{G}_{m, \log }\left(W^{\prime}\right)$ are injective, the map $G_{\log }(W) \rightarrow G_{\log }\left(W^{\prime}\right)$ is injective. Hence we have $\varphi\left(t \bmod \mathbb{G}_{m}\right)=0$.

6.2. Proof of 2.8.2. It is easy to see that $\left(\mathrm{c}^{\prime}\right)$ implies (c). Assume that (c) holds. Let $y, m$ be as in $\left(\mathrm{c}^{\prime}\right)$. Then $m$ belongs to $M_{S} / \mathcal{O}_{S}^{\times}$at each point. Hence $m \in M_{S} / \mathcal{O}_{S}^{\times}$. Let $\widetilde{m}$ be a local lift of $m$ to $M_{S}$. Since $\tilde{m}$ does not belong to the stalk of $\mathcal{O}_{S}^{\times}$at each point, $\alpha(\widetilde{m}) \in \mathcal{O}_{S}$ does not belong to the stalk of $\mathcal{O}_{S}^{\times}$at each point. Then, since the intersection of all prime ideals of a commutative ring coincides with the set of nilpotent elements, $\alpha(\widetilde{m})$ is locally nilpotent.

\section{§7. Computations of Hom and Ext}

Since log abelian varieties are abelian group objects, for the study of them, computations of Hom sheaves and Ext sheaves for abelian group objects related to them are useful. The aim of this section is to prove Theorem 7.3, Theorem 7.4, and Theorem 7.6 concerning such Hom sheaves and Ext sheaves, which will be used in Section 8 and Section 9. 
7.1. Let $S$ be an fs log scheme.

In the analytic theory [6], the notion "admissible pairing" played an important role. We consider it in the algebraic situation.

Let $X$ and $Y$ be finitely generated free $\mathbb{Z}$-modules, let $\mathcal{S}$ be an fs monoid, and let $\langle\rangle:, X \times Y \rightarrow \mathcal{S}^{\text {gp }}$ be a $\mathbb{Z}$-bilinear form. We say $\langle$,$\rangle is admissible$ (or $\mathcal{S}$-admissible for precise) if the following condition is satisfied.

For any face $\sigma$ of $\mathcal{S}$ and any homomorphism $N: \mathcal{S} \rightarrow \mathbb{R}_{>0}$ into the additive monoid $\mathbb{R}_{\geq 0}=\{r \in \mathbb{R} \mid r \geq 0\}$, if we denote the face $\{a \in \sigma \mid$ $N(a)=0\}$ of $\mathcal{S}$ by $\tau$, then the pairing of $\mathbb{R}$-linear spaces

$$
\mathbb{R} \otimes\left(X_{\sigma} / X_{\tau}\right) \times \mathbb{R} \otimes\left(Y_{\sigma} / Y_{\tau}\right) \longrightarrow \mathbb{R} ;(x, y) \longmapsto N(\langle x, y\rangle)
$$

is non-degenerate. Here $X_{\sigma}$ (resp. $Y_{\sigma}$ ) denotes the subgroup of $X$ (resp. $Y$ ) consisting of all elements $x$ (resp. $y$ ) such that $\langle x, Y\rangle$ (resp. $\langle X, y\rangle)$ is contained in $\sigma^{\mathrm{gp}}$, and $X_{\tau}$ (resp. $Y_{\tau}$ ) is defined similarly.

We have (see [6] 1.2.5, and the statement and the proof of [6] 1.2.9)

7.1.1. Let $\langle\rangle:, X \times Y \rightarrow \mathcal{S}^{\text {gp }}$ be an $\mathcal{S}$-admissible pairing and let $\mathcal{S} \rightarrow \mathcal{S}^{\prime}$ be a homomorphism of fs monoids. Then, the induced pairing $X \times Y \rightarrow\left(\mathcal{S}^{\prime}\right)^{\mathrm{gp}}$ is $\mathcal{S}^{\prime}$-admissible. Furthermore, if $\sigma^{\prime}$ is a face of $\mathcal{S}^{\prime}$ and $\sigma$ is the inverse image of $\sigma^{\prime}$ in $\mathcal{S}$, then $X_{\sigma}=X_{\sigma^{\prime}}$.

7.1.2. If there is a homomorphism $\phi: Y \rightarrow X$ whose cokernel is finite such that $\langle\phi(y), z\rangle=\langle\phi(z), y\rangle$ for any $y, z \in Y$ and such that $\langle\phi(y), y\rangle \in \mathcal{S}$ for any $y \in Y$, then $\langle$,$\rangle is admissible.$

Let $S$ be an fs $\log$ scheme, let $X$ and $Y$ be finitely generated free $\mathbb{Z}$ modules, and let $\langle\rangle:, X \times Y \rightarrow\left(\mathbb{G}_{m, \log } / \mathbb{G}_{m}\right)_{S}$ be a $\mathbb{Z}$-bilinear form. We say $\langle$,$\rangle is admissible if the induced pairing X \times Y \rightarrow\left(M_{S}^{\mathrm{gp}} / \mathcal{O}_{S}^{\times}\right)_{\bar{s}}$ is $\left(M_{S} / \mathcal{O}_{S}^{\times}\right)_{\bar{s}}$ admissible for any $s \in S$.

For an admissible pairing $X \times Y \rightarrow\left(\mathbb{G}_{m, \log } / \mathbb{G}_{m}\right)_{S}$, we denote the image of $X \rightarrow \mathcal{H o m}\left(Y, \mathbb{G}_{m, \log } / \mathbb{G}_{m}\right)$ (regarded as a sheaf on $(\mathrm{fs} / S)$ ) by $\bar{X}$, and the image of $Y \rightarrow \mathcal{H o m}\left(X, \mathbb{G}_{m, \log } / \mathbb{G}_{m}\right)$ by $\bar{Y}$. These sheaves $\bar{X}$ and $\bar{Y}$ are inverse images of sheaves on the small étale site of $S$. This is seen from the fact that by the second statement in 7.1.1, for any fs log scheme $U$ over $S$, for any $s \in S$ and any $u \in U$ lying over $s$, the map of stalks $\bar{X}_{\bar{s}} \rightarrow \bar{X}_{\bar{u}}$ is an isomorphism.

An admissible pairing $\langle$,$\rangle is said to be non-degenerate if X \rightarrow$ $\mathcal{H o m}\left(Y, \mathbb{G}_{m, \log } / \mathbb{G}_{m}\right)$ and $Y \rightarrow \mathcal{H o m}\left(X, \mathbb{G}_{m, \log } / \mathbb{G}_{m}\right)$ are injective. That is, an admissible pairing is non-degenerate if and only if $X=\bar{X}$ and $Y=\bar{Y}$. 
Example 7.2. (1) For a $\log 1$-motif of type $(X, Y)$ whose $X$ and $Y$ are constant sheaves and which has a polarization, the associated pairing $X \times Y \rightarrow\left(\mathbb{G}_{m, \log } / \mathbb{G}_{m}\right)_{S}$ is admissible and non-degenerate. This follows from 7.1.2.

(2) Let $q \in M_{S}(S)$, let $X=Y=\mathbb{Z}$, and define the pairing $X \times Y \rightarrow$ $\left(\mathbb{G}_{m, \log } / \mathbb{G}_{m}\right)_{S}$ by $(m, n) \mapsto q^{m n}$. Then this pairing is admissible. We have $\bar{X}=i_{*} \mathbb{Z}$ with $i: S_{q=0} \rightarrow S$, where $S_{q=0}$ denotes the closed subscheme of $S$ defined as the zero of the image of $q$ in $\mathcal{O}_{S}(S)$. Hence this pairing is non-degenerate if and only if the image of $q$ in $\mathcal{O}_{S}$ is locally nilpotent.

In the rest of this section, $S$ denotes an fs log scheme.

Now we state the theorems $7.3,7.4$, and 7.6 whose proofs will be given in $7.7-7.26$.

Theorem 7.3. Let $X$ and $Y$ be finitely generated free $\mathbb{Z}$-modules, and let $\langle\rangle:, X \times Y \rightarrow\left(\mathbb{G}_{m, \log } / \mathbb{G}_{m}\right)_{S}$ be a non-degenerate admissible pairing.

Let $G$ be a commutative group scheme over the underlying scheme of $S$ having an exact sequence $0 \rightarrow T \rightarrow G \rightarrow B \rightarrow 0$ with $T$ a torus over $S$ and $B$ an abelian scheme over $S$. Assume that we are given an isomorphism $T \cong$ $\mathcal{H o m}\left(X, \mathbb{G}_{m}\right)$. Let $T_{\log }^{(Y)}=\mathcal{H o m}\left(X, \mathbb{G}_{m, \log }\right)^{(Y)} \subset T_{\log }=\mathcal{H o m}\left(X, \mathbb{G}_{m, \log }\right)$ (resp. $\left.G_{\log }^{(Y)} \subset G_{\log }\right)$ be the inverse image of $\mathcal{H o m}\left(X, \mathbb{G}_{m, \log } / \mathbb{G}_{m}\right)^{(Y)} \subset$ $\mathcal{H o m}\left(X, \mathbb{G}_{m, \log } / \mathbb{G}_{m}\right) \cong T_{\log } / T \cong G_{\log } / G$.

(1) Let $H$ be a commutative group scheme over $S$ and regard $H$ as a sheaf of abelian groups on $(\mathrm{fs} / S)$ in the natural way. Then we have canonical isomorphisms

$$
\mathcal{H o m}\left(G_{\log }^{(Y)}, H\right) \cong \mathcal{H} \operatorname{Hom}(B, H), \quad \mathcal{E} x t\left(G_{\log }^{(Y)}, H\right) \cong \mathcal{E} x t(B, H)
$$

induced by the projection $G_{\log }^{(Y)} \rightarrow B$. We have

$$
\mathcal{H o m}\left(G_{\log }^{(Y)} / G, H\right)=0
$$

and an isomorphism

$$
\mathcal{E} x t\left(G_{\log }^{(Y)} / G, H\right) \cong \mathcal{H o m}(T, H)
$$

given as the boundary map associated to the exact sequence $0 \rightarrow T \rightarrow$ $T_{\log }^{(Y)} \rightarrow G_{\log }^{(Y)} / G \rightarrow 0$. 
(2) We have

$$
\begin{gathered}
\mathcal{H o m}\left(T_{\log }^{(Y)}, \mathbb{G}_{m, \log }\right) \cong X \\
\mathcal{H o m}\left(T_{\log }^{(Y)} / T, \mathbb{G}_{m, \log }\right)=0, \mathcal{E} x t\left(T_{\log }^{(Y)} / T, \mathbb{G}_{m, \log }\right)=0, \mathcal{E} x t\left(T_{\log }^{(Y)}, \mathbb{G}_{m, \log }\right)=0
\end{gathered}
$$

(3) Let $G^{\prime}$ be a commutative group scheme over the underlying scheme of $S$ having an exact sequence $0 \rightarrow T^{\prime} \rightarrow G^{\prime} \rightarrow B^{\prime} \rightarrow 0$ with $T^{\prime}$ a torus over $S$ and $B^{\prime}$ an abelian scheme over $S$. Let $X^{\prime}=\mathcal{H o m}\left(T^{\prime}, \mathbb{G}_{m}\right)$. Then

$$
\begin{gathered}
\mathcal{H o m}\left(G, G^{\prime}\right) \stackrel{\cong}{\longrightarrow} \mathcal{H o m}\left(G_{\log }^{(Y)}, G_{\log }^{\prime}\right), \\
\mathcal{H o m}\left(X^{\prime}, X\right) \stackrel{\cong}{\longrightarrow} \operatorname{Hom}\left(G_{\log }^{(Y)} / G, G_{\log }^{\prime} / G^{\prime}\right) .
\end{gathered}
$$

TheOREm 7.4. Let $\left[Y \rightarrow G_{\log }\right]$ be a log 1-motif over $S$ of type $(X, Y)$ such that the induced pairing $X \times Y \rightarrow \mathbb{G}_{m, \log } / \mathbb{G}_{m}$ is admissible and nondegenerate (see Remark 7.5 (1) below), and let $\left[X \rightarrow G_{\log }^{*}\right]$ be its dual. Let $A=G_{\log }^{(Y)} / Y$. Then we have:

(1) $\mathcal{E} x t(A, \mathbb{Z}) \cong \mathcal{H o m}(Y, \mathbb{Z})$;

(2) $\mathcal{E} x t\left(A, \mathbb{G}_{m}\right) \cong G^{*}$

(3) $\mathcal{E} x t\left(A, \mathbb{G}_{m, \log }\right) \cong G_{\log }^{*} / X$

(4) The sheaves $\mathcal{H o m}(A, \mathbb{Z}), \mathcal{H o m}\left(A, \mathbb{G}_{m}\right), \mathcal{H o m}\left(A, \mathbb{G}_{m, \log }\right)$ are zero.

Remark 7.5. (1) In the above, we say a pairing with locally constant $X$ and $Y$ is admissible and non-degenerate if it is locally so in the sense of 7.1 .

(2) From Part III, we plan to consider the dual of a log abelian variety. Recall that the dual abelian scheme $A^{*}$ of an abelian scheme $A$ is $\mathcal{E} x t\left(A, \mathbb{G}_{m}\right)$. In the case of a log abelian variety with constant degeneration $A=G_{\log }^{(Y)} / Y$ associated to a pointwise polarizable $\log 1$-motif $\left[Y \rightarrow G_{\log }^{(Y)}\right]$, the dual $A^{*}$ of $A$ is given by $A^{*}=\left(G_{\log }^{*}\right)^{(X)} / X$. Thus, in this case, Theorem 7.4 (3) shows that $A^{*}$ is embedded in $\mathcal{E} x t\left(A, \mathbb{G}_{m, \text { log }}\right)$. Our plan is that $A^{*}$ of a general log abelian variety $A$ should be defined as a certain subgroup sheaf of $\mathcal{E} x t\left(A, \mathbb{G}_{m, \log }\right)$.

(3) In Theorem 7.3 and Theorem 7.4, we assumed that the admissible pairing $X \times Y \rightarrow\left(\mathbb{G}_{m, \log } / \mathbb{G}_{m}\right)_{S}$ is non-degenerate. The log abelian varieties to which these results are directly applied are those with constant degeneration. 
Theorem 7.6. Let $X, Y, X^{\prime}, Y^{\prime}$ be finitely generated free $\mathbb{Z}$-modules and let

$$
\langle,\rangle: X \times Y \longrightarrow\left(\mathbb{G}_{m, \log } / \mathbb{G}_{m}\right)_{S}, \quad\langle,\rangle^{\prime}: X^{\prime} \times Y^{\prime} \longrightarrow\left(\mathbb{G}_{m, \log } / \mathbb{G}_{m}\right)_{S}
$$

be admissible pairings. (They need not be non-degenerate.)

(1) Let $F$ be the sheaf of pairs $(u, v)$ of homomorphisms $u: \bar{X}^{\prime} \rightarrow$ $\bar{X}, v: \bar{Y} \rightarrow \bar{Y}^{\prime}$ such that $\left\langle u\left(x^{\prime}\right), y\right\rangle=\left\langle x^{\prime}, v(y)\right\rangle^{\prime}$ for any $x^{\prime} \in \bar{X}^{\prime}, y \in$ $\bar{Y}$. (Here $\bar{X}^{\prime}$ denotes the image of $X^{\prime} \rightarrow \mathcal{H o m}\left(Y^{\prime}, \mathbb{G}_{m, \log } / \mathbb{G}_{m}\right)$ and $\bar{Y}^{\prime}$ is defined similarly. $)$ Let $H=\mathcal{H o m}\left(X, \mathbb{G}_{m, \log } / \mathbb{G}_{m}\right)^{(Y)} / \bar{Y}, H^{\prime}=\mathcal{H o m}\left(X^{\prime}\right.$, $\left.\mathbb{G}_{m, \log } / \mathbb{G}_{m}\right)^{\left(Y^{\prime}\right)} / \bar{Y}^{\prime}$. Then the canonical homomorphism $F \rightarrow \mathcal{H o m}\left(H, H^{\prime}\right)$ is an isomorphism.

(2) Assume that we are given an isomorphism

$$
h: \mathcal{H o m}\left(X, \mathbb{G}_{m, \log } / \mathbb{G}_{m}\right)^{(Y)} / \bar{Y} \cong \mathcal{H} \operatorname{Hom}\left(X^{\prime}, \mathbb{G}_{m, \log } / \mathbb{G}_{m}\right)^{\left(Y^{\prime}\right)} / \bar{Y}^{\prime} .
$$

Then there is a unique pair of isomorphisms $\bar{X} \simeq \bar{X}^{\prime}$ and $\bar{Y} \simeq \bar{Y}^{\prime}$ which is compatible with $\bar{X} \times \bar{Y} \rightarrow\left(\mathbb{G}_{m, \log } / \mathbb{G}_{m}\right)_{S}$ and $\bar{X}^{\prime} \times \bar{Y}^{\prime} \rightarrow\left(\mathbb{G}_{m, \log } / \mathbb{G}_{m}\right)_{S}$ and which induces $h$.

Remark 7.6.1. (1) In 7.6 (1), the canonical homomorphism $F \rightarrow$ $\mathcal{H o m}\left(H, H^{\prime}\right)$ is defined since any section of $\mathcal{H o m}\left(X, \mathbb{G}_{m, \log } / \mathbb{G}_{m}\right)^{(Y)}$ factors through $X \rightarrow \bar{X}$. The last fact is shown as follows. Take a section $\varphi$ of $\operatorname{Hom}\left(X, \mathbb{G}_{m, \log } / \mathbb{G}_{m}\right)^{(Y)}$. If $x \in X$, locally on $S$, there exist $y, y^{\prime} \in Y$ such that $\langle x, y\rangle|\varphi(x)|\left\langle x, y^{\prime}\right\rangle$ in $M_{S}^{\mathrm{gp}} / \mathcal{O}_{S}^{\times}$. If $x$ belongs to the kernel of $X \rightarrow \bar{X}$, since the pairing $X \times Y \rightarrow \mathbb{G}_{m, \log } / \mathbb{G}_{m}$ factors as $X \times Y \rightarrow \bar{X} \times \bar{Y} \rightarrow \mathbb{G}_{m, \log } / \mathbb{G}_{m},\langle x, y\rangle$ and $\left\langle x, y^{\prime}\right\rangle$ vanish in $M_{S}^{\mathrm{gp}} / \mathcal{O}_{S}^{\times}$. Hence $\varphi(x)$ vanishes in $M_{S}^{\mathrm{gp}} / \mathcal{O}_{S}^{\times}$.

(2) In 7.6, (2) is a corollary of (1).

The rest of Section 7 is devoted to the proofs of the theorems 7.3, 7.4, and 7.6.

For the proofs of these theorems, we use the following Proposition 7.9. First we make a preparation to state it.

7.7. Let $X, Y$ be finitely generated free $\mathbb{Z}$-modules, and let $\langle\rangle:, X \times$ $Y \rightarrow\left(\mathbb{G}_{m, \log } / \mathbb{G}_{m}\right)_{S}$ be an admissible pairing. Then étale locally on $S$, there exist an fs chart $\mathcal{S} \rightarrow M_{S}$ and an admissible pairing $X \times Y \rightarrow \mathcal{S}^{\mathrm{gp}}$ which induces the original pairing. 
Assume that we are given such $\mathcal{S} \rightarrow M_{S}$ and $X \times Y \rightarrow \mathcal{S}^{\text {gp }}$ (we denote the last pairing also by $\langle\rangle$,$) . Let C$ be the subcone of $\operatorname{Hom}(\mathcal{S}, \mathbb{N}) \times$ $\operatorname{Hom}(X, \mathbb{Z})$ defined as

$$
C=\left\{(N, l) \mid l\left(X_{\operatorname{ker}(N)}\right)=0\right\}
$$

(cf. [6] 3.4). By a finitely generated subcone of $C$, we mean a finitely generated submonoid $\Delta$ of the additive monoid $C$ such that for any $a \in C$ satisfying $n a \in \Delta$ for some $n \geq 1$, we have $a \in \Delta$.

For a finitely generated subcone $\Delta$ of $C$, define subsheaves

$$
V(\Delta) \subset \mathcal{H o m}\left(X, \mathbb{G}_{m, \log }\right)^{(Y)}, \quad \bar{V}(\Delta) \subset \mathcal{H o m}\left(X, \mathbb{G}_{m, \log } / \mathbb{G}_{m}\right)^{(Y)}
$$

as follows ([6] 3.5). For an fs log scheme $U$ over $S$,

$$
\begin{array}{r}
\bar{V}(\Delta)(U)=\left\{\varphi \in \mathcal{H o m}\left(X, \mathbb{G}_{m, \log } / \mathbb{G}_{m}\right)(U) \mid\right. \\
\left.\mu \varphi(x) \in M_{U} / \mathcal{O}_{U}^{\times} \text {for any }(\mu, x) \in \Delta^{\vee}\right\},
\end{array}
$$

where $\Delta^{\vee}$ is the dual cone of $\Delta$ in $\mathcal{S}^{\mathrm{gp}} \times X$, that is,

$$
\Delta^{\vee}=\left\{(\mu, x) \in \mathcal{S}^{\mathrm{gp}} \times X \mid N(\mu)+l(x) \geq 0 \text { for all }(N, l) \in \Delta\right\} .
$$

Let $V(\Delta)$ be the inverse image of $\bar{V}(\Delta)$ in $\mathcal{H o m}\left(X, \mathbb{G}_{m, \log }\right)^{(Y)}$. Then $V(\Delta)$ is represented by the fiber product $S \times{ }_{\operatorname{Spec}(\mathbb{Z}[\mathcal{S}])} \operatorname{Spec}\left(\mathbb{Z}\left[\Delta^{\vee}\right]\right)$.

If $\Delta^{\prime}$ is also a finitely generated subcone of $C$ such that $\Delta \subset \Delta^{\prime}$, then $V(\Delta) \subset V\left(\Delta^{\prime}\right)$ and $\bar{V}(\Delta) \subset \bar{V}\left(\Delta^{\prime}\right)$.

We have

$$
\mathcal{H o m}\left(X, \mathbb{G}_{m, \log }\right)^{(Y)}=\bigcup_{\Delta} V(\Delta), \quad \mathcal{H o m}\left(X, \mathbb{G}_{m, \log } / \mathbb{G}_{m}\right)^{(Y)}=\bigcup_{\Delta} \bar{V}(\Delta),
$$

where $\Delta$ ranges over all finitely generated subcones of $C$. (The proofs are the same as the analytic version given in [6] 3.5.) The unit section of $\mathcal{H o m}\left(X, \mathbb{G}_{m, \log }\right)^{(Y)}\left(\operatorname{resp} . \mathcal{H o m}\left(X, \mathbb{G}_{m, \log } / \mathbb{G}_{m}\right)^{(Y)}\right)$ is contained in $V(\Delta)$ (resp. $\bar{V}(\Delta))$ if $\mathcal{S}^{\vee} \times\{1\} \subset \Delta$.

EXAMPLE 7.8. Let $K$ and $q$ be as in Section 1. Let $S=\operatorname{Spec}\left(O_{K} / m_{K}^{n}\right)$, let $\mathcal{S}=\mathbb{N}$ with a homomorphism $\mathcal{S} \rightarrow M_{S}$ which sends 1 to the class of a prime element of $K, X=Y=\mathbb{Z}, X \times Y \rightarrow \mathcal{S}^{\text {gp }}=\mathbb{Z}$ the pairing $(m, n) \mapsto m n e$, where $e=\operatorname{ord}_{K}(q)$. Then $V_{\alpha, n}$ in 1.5 coincides with $V(\Delta)$, where $\Delta \subset \operatorname{Hom}(\mathcal{S}, \mathbb{N}) \times \operatorname{Hom}(X, \mathbb{Z}) \subset \mathbb{Z}^{2}$ is the intersection of $\mathbb{Z}^{2}$ and the cone $\left\{a(1, e \alpha)+b\left(1, e \alpha^{\prime}\right) \mid a, b \in \mathbb{Q}, a, b \geq 0\right\}$ in $\mathbb{Q}^{2}$. (The $N_{\alpha}$ there is $\Delta^{\vee}$ here.) 
Proposition 7.9. Assume that we are given an $f$ s chart $\mathcal{S} \rightarrow M_{S}$, finitely generated free $\mathbb{Z}$-modules $X, Y$, and an admissible pairing $\langle\rangle:, X \times$ $Y \rightarrow \mathcal{S}^{\mathrm{gp}}$. Let $\Delta$ be a finitely generated subcone of $C$.

(1) There exists a finitely generated subcone $\Delta^{\prime}$ of $C$ containing $\Delta$ and satisfying the following conditions (i) and (ii).

(i) If $(\mu, x) \in\left(\Delta^{\prime}\right)^{\vee}\left(\mu \in \mathcal{S}^{\mathrm{gp}}, x \in X\right)$, then $\mu \in \mathcal{S}$.

(ii) Let $\sigma$ be a face of $\mathcal{S}$. If $(\mu, x) \in\left(\Delta^{\prime}\right)^{\vee}$ and $x \notin X_{\sigma}$, then there exist $\mu^{\prime}, \mu^{\prime \prime} \in \mathcal{S}$ such that $\mu=\mu^{\prime} \mu^{\prime \prime}$ and such that $\left(\mu^{\prime}, x\right) \in \Delta^{\vee}$ and $\mu^{\prime \prime} \notin \sigma$.

(2) Let $\Delta^{\prime}$ be a finitely generated subcone of $C$ as in (1). Let $f: V(\Delta) \rightarrow$ $V\left(\Delta^{\prime}\right)$ be the inclusion morphism. Then

$$
f^{-1}\left(M_{V\left(\Delta^{\prime}\right)}^{\mathrm{gp}} / \mathcal{O}_{V\left(\Delta^{\prime}\right)}^{\times}\right) \simeq M_{S}^{\mathrm{gp}} / \mathcal{O}_{S}^{\times} \oplus \bar{X}
$$

Here we denote the inverse image of $M_{S}^{\mathrm{gp}} / \mathcal{O}_{S}^{\times}$on $V(\Delta)$ also by $M_{S}^{\mathrm{gp}} / \mathcal{O}_{S}^{\times}$.

(3) Assume that the pairing $X \times Y \rightarrow M_{S}^{\mathrm{gp}} / \mathcal{O}_{S}^{\times}$is non-degenerate. Assume that $S$ is quasi-compact. Then there exists a finitely generated subcone $\Delta^{\prime}$ of $C$ containing $\Delta \cup\left(\mathcal{S}^{\vee} \times\{1\}\right)$ such that the morphism of underlying schemes of the inclusion morphism $V(\Delta) \rightarrow V\left(\Delta^{\prime}\right)$ coincides with the morphism of underlying schemes of the composition $V(\Delta) \rightarrow S \rightarrow V\left(\Delta^{\prime}\right)$, where the first arrow is the structural morphism and the second arrow is the unit section of $\mathcal{H o m}\left(X, \mathbb{G}_{m, \log }\right)^{(Y)}$.

(1) of Proposition 7.9 is the same as Proposition 3.4.8 of [6].

In Proposition 7.9 (3), in the case where the underlying scheme of $S$ is the Spec of a field, any $\Delta^{\prime}$ satisfying the conditions (i) and (ii) has the desired property. This fact is proved by the same method as [6] Proposition 3.5.6 (1) (i) which treats the analytic situation.

7.10. We prove Proposition 7.9 (2). For a finitely generated subcone $\Delta$ of $C$, we have a chart $\Delta^{\vee} \rightarrow M_{V(\Delta)}$. This induces a homomorphism $\mathcal{S}^{\mathrm{gp}} \oplus X=\left(\Delta^{\vee}\right)^{\mathrm{gp}} \rightarrow M_{V(\Delta)}^{\mathrm{gp}}$. We claim

7.10.1. The induced homomorphism $\varphi: X \rightarrow M_{V(\Delta)}^{\mathrm{gp}} / \mathcal{O}_{V(\Delta)}^{\times}$factors through $\bar{X}$.

Proof. Since $V(\Delta) \subset \mathcal{H o m}\left(X, \mathbb{G}_{m, \log }\right)^{(Y)}$, this reduces to the fact proved in Remark 7.6.1 (1). 
Now assume that $\Delta^{\prime}$ satisfies the conditions (i) and (ii). Let $v \in V\left(\Delta^{\prime}\right)$, and let $s$ be the image of $v$ in $S$. Let $\sigma$ be the kernel of $\mathcal{S} \rightarrow\left(M_{S} / \mathcal{O}_{S}^{\times}\right)_{\bar{s}}$ and let $\tau$ be the kernel of $\left(\Delta^{\prime}\right)^{\vee} \rightarrow\left(M_{V\left(\Delta^{\prime}\right)} / \mathcal{O}_{V\left(\Delta^{\prime}\right)}^{\times}\right) \bar{v}$. Since $\mathcal{S} \rightarrow M_{S}$ and $\left(\Delta^{\prime}\right)^{\vee} \rightarrow M_{V\left(\Delta^{\prime}\right)}$ are charts, we have

$$
\begin{gathered}
\mathcal{S}^{\mathrm{gp}} / \sigma^{\mathrm{gp}} \cong\left(M_{S}^{\mathrm{gp}} / \mathcal{O}_{S}^{\times}\right)_{\bar{s}}, \\
\left(\mathcal{S}^{\mathrm{gp}} \oplus X\right) / \tau^{\mathrm{gp}}=\left(\left(\Delta^{\prime}\right)^{\vee}\right)^{\mathrm{gp}} / \tau^{\mathrm{gp}} \cong\left(M_{V\left(\Delta^{\prime}\right)}^{\mathrm{gp}} / \mathcal{O}_{V\left(\Delta^{\prime}\right)}^{\times}\right)_{\bar{v}}
\end{gathered}
$$

Furthermore, $\bar{X}_{\bar{v}}=X / X_{\sigma}$. Hence it is sufficient to prove that if $v$ is the image of some point $u \in V(\Delta)$, then $\tau^{\mathrm{gp}}=\sigma^{\mathrm{gp}} \times X_{\sigma}$. By 7.10.1, we have $\tau^{\mathrm{gp}} \supset \sigma^{\mathrm{gp}} \times X_{\sigma}$. To prove the converse inclusion, it is sufficient to prove $\tau \subset$ $\sigma^{\mathrm{gp}} \times X_{\sigma}$. Let $(\mu, x) \in\left(\Delta^{\prime}\right)^{\vee}$. By the condition (i), we have $\mu \in \mathcal{S}$. If $x \notin X_{\sigma}$, then by the condition (ii), $\mu=\mu^{\prime} \mu^{\prime \prime}$ for some $\mu^{\prime}, \mu^{\prime \prime} \in \mathcal{S}$ such that $\left(\mu^{\prime}, x\right) \in$ $\Delta^{\vee}$ and $\mu^{\prime \prime} \notin \sigma$. This shows that the image of $(\mu, x)$ in $\left(M_{V(\Delta)} / \mathcal{O}_{V(\Delta)}^{\times}\right) \bar{u}$ does not vanish. Then the image of $(\mu, x)$ in $\left(M_{V\left(\Delta^{\prime}\right)} / \mathcal{O}_{V\left(\Delta^{\prime}\right)}^{\times}\right)_{v}$ does not vanish. Hence $(\mu, x) \notin \tau$. Hence if $(\mu, x) \in \tau$, we have $x \in X_{\sigma} \subset \tau^{\mathrm{gp}}$ and hence the image of $\mu$ belongs to $\tau$. Since the kernel of $\left(M_{S} / \mathcal{O}_{S}^{\times}\right)_{\bar{s}} \rightarrow\left(M_{V\left(\Delta^{\prime}\right)} / \mathcal{O}_{V\left(\Delta^{\prime}\right)}^{\times}\right) \bar{v}$ is trivial, we have $\mu \in \sigma$. This completes the proof of 7.9 (2).

In the following 7.11-7.12, we give preliminaries for the proof of Proposition 7.9 (3). We prove Proposition 7.9 (3) in 7.13-7.16.

7.11. (This is 3.4 .9 of [6].) Let $X$ and $Y$ be finitely generated free $\mathbb{Z}$ modules, let $\mathcal{S}$ be an fs monoid, and let $\langle\rangle:, X \times Y \rightarrow \mathcal{S}^{\text {gp }}$ be an admissible pairing. For each face $\sigma$ of $\mathcal{S}$, fix an element $s_{\sigma}$ of the interior of $\sigma$ and fix a $\mathbb{Z}$-basis $\left(e_{\sigma, i}\right)_{i}$ of $X_{\sigma}$. For an integer $a \geq 0$, let

$$
\begin{aligned}
C(a):=\{(N, l) \in \operatorname{Hom}(\mathcal{S}, \mathbb{N}) \times \operatorname{Hom}(X, \mathbb{Z}) \mid \\
\left.a \cdot N\left(s_{\sigma}\right) \geq\left|l\left(e_{\sigma, i}\right)\right| \text { for all } \sigma \text { and } i\right\} .
\end{aligned}
$$

The following is Lemma 3.4.10 of [6].

Lemma 7.12. (1) $C(a)$ is a finitely generated subcone of $C$.

(2) $\bigcup_{a} C(a)=C$.

Lemma 7.13. Let the assumptions be as in Proposition 7.9 (3). Let $\Theta$ be the set of all faces $\sigma$ of $\mathcal{S}$ for which there exists a point $s$ of $S$ such that $\sigma$ coincides with the kernel of $\mathcal{S} \rightarrow M_{S, \bar{s}} / \mathcal{O}_{S, \bar{s}}^{\times}$.

(1) If $\sigma \in \Theta$, then $X_{\sigma}=\{1\}$.

(2) If $N \in \operatorname{Hom}(\mathcal{S}, \mathbb{N})$ and $\operatorname{Ker}(N) \in \Theta$, then we have $(N, l) \in C$ for any $l \in \operatorname{Hom}(X, \mathbb{Z})$. 
Proof. (2) follows from (1). We prove (1). Let $s$ be a point of $S$ such that $\sigma$ is the kernel of $\mathcal{S} \rightarrow M_{S, \bar{s}} / \mathcal{O}_{S, \bar{s}}^{\times}$. Then $X_{\sigma}$ is contained in the kernel of $X \rightarrow \operatorname{Hom}\left(Y, M_{S, \bar{s}}^{\mathrm{gp}} / \mathcal{O}_{S, \bar{s}}^{\times}\right)$and hence should be $\{1\}$ because the pairing is nondegenerate.

Lemma 7.14. For each $\sigma \in \Theta$, fix a homomorphism $N_{\sigma}: \mathcal{S} \rightarrow \mathbb{N}$ such that $\operatorname{Ker}\left(N_{\sigma}\right)=\sigma$. Let $\mu \in \mathcal{S}$, and assume that $N_{\sigma}(\mu)>0$ for any $\sigma \in \Theta$. Then the image of $\mu$ in $\Gamma\left(S, \mathcal{O}_{S}\right)$ is nilpotent.

Proof. Since the intersection of all prime ideals of a commutative ring coincides with the set of nilpotent elements, and since $S$ is quasi-compact, it is sufficient to prove that for any $s \in S$, the image of $\mu$ in $M_{S, \bar{s}} / \mathcal{O}_{S, \bar{s}}^{\times}$is non-trivial. Let $\sigma \in \Theta$ be the kernel of $\mathcal{S} \rightarrow M_{S, \bar{s}} / \mathcal{O}_{S, \bar{s}}^{\times}$. Since $\mathcal{S} \rightarrow M_{S}$ is a chart, we have $\mathcal{S} / \sigma \stackrel{\cong}{\rightarrow} M_{S, \bar{s}} / \mathcal{O}_{S, \bar{s}}^{\times}$. Since $\mu$ does not belong to $\operatorname{Ker}\left(N_{\sigma}\right)=\sigma$, this implies that the image of $\mu$ in $M_{S . \bar{s}} / \mathcal{O}_{S, \bar{s}}^{\times}$is non-trivial.

The following Lemma 7.15 is a refinement of Lemma 7.14:

LEMMA 7.15. Let

$$
I:=\left\{\mu \in \mathcal{S} \mid \text { the image of } \mu \text { in } \Gamma\left(S, \mathcal{O}_{S}\right) \text { is nilpotent }\right\} .
$$

Then for each $n \geq 1$, the following holds for any sufficiently large integer $m:$ If $\mu \in \mathcal{S}$ and if $N_{\sigma}(\mu) \geq m$ for any $\sigma \in \Theta$, then $\mu$ belongs to the $n$-th power $I^{n}$ of the ideal $I$ of $\mathcal{S}$.

Here an ideal $J$ of $\mathcal{S}$ means a subset $J$ of $\mathcal{S}$ such that $a \mu \in J$ for any $a \in \mathcal{S}$ and $\mu \in J$. For $n \geq 1$, the $n$-th power of the ideal $J$ means the subset $\left\{\prod_{i=1}^{n} \mu_{i} \mid \mu_{i} \in J\right\}$.

Proof. We may assume that $I$ is not empty. An ideal of $\mathcal{S}$ is finitely generated. (This follows from the fact that the $\operatorname{ring} \mathbb{Z}[\mathcal{S}]$ is Noetherian.) Let $J$ be a finite subset of $I$ which generates the ideal $I$, and let $b$ be the integer $\max \left(\bigcup_{\sigma \in \Theta} N_{\sigma}(J)\right)$. We show that any $m \geq b n$ has the property stated in Lemma 7.15. We use the induction on $n$. If $n=1$, then Lemma 7.14 in fact shows that any $m \geq 1$ has the property stated in Lemma 7.15. Assume $n \geq 2$, and let $\mu$ be an element of $\mathcal{S}$ such that $N_{\sigma}(\mu) \geq b n$ for any $\sigma \in \Theta$. Then $\mu \in I$ by Lemma 7.14. Hence $\mu=\mu^{\prime} \mu^{\prime \prime}$ for some $\mu^{\prime} \in \mathcal{S}$ and for $\mu^{\prime \prime} \in J$. We have

$$
N_{\sigma}\left(\mu^{\prime}\right)=N_{\sigma}(\mu)-N_{\sigma}\left(\mu^{\prime \prime}\right) \geq b n-b=b(n-1) .
$$

By induction on $n$, we have $\mu^{\prime} \in I^{n-1}$. Hence $\mu \in I^{n}$. 
7.16. We now prove Proposition 7.9 (3).

For each face $\sigma$ of $\mathcal{S}$, fix an element $s_{\sigma}$ of the interior of $\sigma$ and a basis $\left(e_{\sigma, i}\right)_{i}$ of $X_{\sigma}$. For an integer $a \geq 0$, let $C(a)$ be as in 7.11. Fix also a finite subset $B$ of $\operatorname{Hom}(X, \mathbb{Z})$ which generates the $\mathbb{Z}$-module $\operatorname{Hom}(X, \mathbb{Z})$. By Lemma $7.13(2)$ and by $\bigcup_{a} C(a)=C(7.12(2))$, the following condition $(*)$ is satisfied if $a$ is large enough:

$$
\left(N_{\sigma}, l\right) \in C(a) \text { for any } \sigma \in \Theta \text { and } l \in B \text {. }
$$

Since $\Delta \subset C(a)$ for some of such an $a>0$, we may assume $\Delta=C(a)$ for some $a>0$ satisfying $(*)$.

Fix a finite set of generators $\left\{\left(N_{i}, l_{i}\right)\right\}_{1 \leq i \leq r}$ of $\Delta=C(a)$, and fix also a finite set of generators $J$ of the ideal $I$ of $\mathcal{S}$. Fix $k \geq 1$ such that $\alpha\left(I^{k}\right)=\{0\}$. Here $\alpha$ is the chart. Now let $m \geq 1$ be a sufficiently large integer satisfying the following (i) and (ii):

(i) If $\mu \in \mathcal{S}$ and if $N_{\sigma}(\mu) \geq m$ for any $\sigma \in \Theta$, then $\mu \in I^{k}$ (Note that (i) is satisfied if $m$ is large enough by Lemma 7.15);

(ii) $m>k \cdot \max \left\{N_{i}(\mu) \mid 1 \leq i \leq r, \mu \in J\right\}$.

We prove that $\Delta^{\prime}=C(\mathrm{am})$ has the property stated in 7.9 (3). Let $(\mu, x) \in\left(\Delta^{\prime}\right)^{\vee}$. It is sufficient to prove the following (a) and (b):

(a) $\mu \in \mathcal{S}$;

(b) $\alpha(\mu)=\alpha((\mu, x))$ on $V(\Delta)$.

First, (a) follows from $N_{i}(\mu) \geq\left|m l_{i}(x)\right| \geq 0$ for all $i$. (Here we use $\left(N_{i}, \pm m l_{i}\right) \in C(\mathrm{am})=\Delta^{\prime}$.) We prove (b). If $x=1$, then $\mu=(\mu, x)$ and so there is no problem. We prove that if $x \neq 1$, then there are elements $\mu^{\prime}$ and $\mu^{\prime \prime}$ of $\mathcal{S}$ such that $\mu=\mu^{\prime} \mu^{\prime \prime}, \alpha\left(\mu^{\prime \prime}\right)=0$, and $\left(\mu^{\prime}, x\right) \in \Delta^{\vee}$. Then on $V(\Delta)$, we will have

$$
\begin{aligned}
& \alpha((\mu, x))=\alpha\left(\left(\mu^{\prime}, x\right) \mu^{\prime \prime}\right)=\alpha\left(\left(\mu^{\prime}, x\right)\right) \alpha\left(\mu^{\prime \prime}\right)=0, \\
& \alpha(\mu)=\alpha\left(\mu^{\prime} \mu^{\prime \prime}\right)=\alpha\left(\mu^{\prime}\right) \alpha\left(\mu^{\prime \prime}\right)=0 .
\end{aligned}
$$

Assume $x \neq 1$. Take $l \in B$ such that $l(x) \neq 0$. For any $\sigma \in \Theta$, $\left(N_{\sigma}, l\right) \in C(a)$ by $(*)$ and hence $\left(N_{\sigma}, m l\right) \in C(a m)$. Hence

$$
N_{\sigma}(\mu) \geq m|l(x)| \geq m .
$$

By (i), we have $\mu \in I^{k}$. Hence $\mu=\mu^{\prime} \cdot \prod_{j=1}^{k} \mu_{j}$ for some $\mu^{\prime} \in \mathcal{S}$ and $\mu_{j} \in J$. Let $\mu^{\prime \prime}=\prod_{j=1}^{k} \mu_{j}$. Then $\alpha\left(\mu^{\prime \prime}\right)=0$. We prove $\left(\mu^{\prime}, x\right) \in \Delta^{\vee}$. It is sufficient 
to prove $N_{i}\left(\mu^{\prime}\right)+l_{i}(x) \geq 0$ for $1 \leq i \leq r$. If $l_{i}(x)=0$, we have nothing to prove. If $l_{i}(x) \neq 0$,

$$
\begin{aligned}
N_{i}\left(\mu^{\prime}\right)+l_{i}(x)= & N_{i}(\mu)-\sum_{j=1}^{k} N_{i}\left(\mu_{j}\right)+l_{i}(x) \\
\geq & m\left|l_{i}(x)\right|-k \cdot \max \left(\left\{N_{i}\left(\mu_{j}\right)\right\}_{i, j}\right)+l_{i}(x) \\
& \quad\left(\text { this follows from }\left(N_{i}, \pm m l_{i}\right) \in \Delta^{\prime}\right) \\
\geq & \left.m-1-k \cdot \max \left(\left\{N_{i}\left(\mu_{j}\right)\right\}_{i, j}\right) \geq 0 \quad \text { (by (ii) }\right) .
\end{aligned}
$$

7.17. We now start the proof of Theorem 7.3. We first prove that the sheaves $\mathcal{H o m}\left(T_{\log }^{(Y)}, H\right), \mathcal{H o m}\left(T_{\log }^{(Y)} / T, \mathbb{G}_{m, \log }\right), \mathcal{E} x t\left(T_{\log }^{(Y)}, H\right), \mathcal{E} x t\left(T_{\log }^{(Y)}\right.$, $\left.\mathbb{G}_{m, \log }\right)$ are zero. Without a loss of generality, let the assumptions be as in Proposition 7.9 (3). For a finitely generated subcone $\Delta$ of $C$ which contains $\mathcal{S}^{\vee} \times\{1\}$, let $S_{\Delta}$ be the fs $\log$ scheme over $S$ whose underlying scheme over $S$ is that of $S$ and which is endowed with the inverse image of the log structure of $V(\Delta)$ under the unit section $S \rightarrow V(\Delta)$ of $T_{\log }^{(Y)}$. Then by $7.9(3), T_{\log }^{(Y)}$ is the union of subsheaves represented by $S_{\Delta}$, where $\Delta$ ranges over all finitely generated subcones of $C$.

Proof of $\mathcal{H o m}\left(T_{\log }^{(Y)}, H\right)=0$. Let $h$ be a homomorphism $T_{\log }^{(Y)} \rightarrow H$. It is sufficient to prove that the restriction of $h$ to each $S_{\Delta}$ is trivial. Since $\operatorname{Mor}_{S}\left(S_{\Delta}, H\right)$ is identified with the set of $S$-morphisms $S \rightarrow H$, and since the origin $S \rightarrow S_{\Delta}$ of $T_{\log }$ is sent to the origin of $H$ by $h$, the morphism $S_{\Delta} \rightarrow H$ induced by $h$ is trivial.

Proof of $\mathcal{H o m}\left(T_{\log }^{(Y)} / T, \mathbb{G}_{m, \log }\right)=0$. Let $h$ be a homomorphism $T_{\log }^{(Y)} \rightarrow$ $\mathbb{G}_{m, \log }$ and assume that $h$ kills $T$. We show that the restriction of $h$ to each $S_{\Delta}$ is trivial. By Proposition $7.9(2)$, if we replace $\Delta$ by a sufficiently big finitely generated subcone of $C$, then the set $\Gamma\left(S_{\Delta}, M_{S_{\Delta}}^{\mathrm{gp}}\right)$ is identified with $\Gamma\left(S, M_{S}^{\mathrm{gp}} \times X\right)$. Let $(\mu, x) \in \Gamma\left(S, M_{S}^{\mathrm{gp}} \times X\right)$ be the element corresponding to the restriction of $h$ to $S_{\Delta}$. The pull back $\mu$ of $(\mu, x)$ to the origin $S \rightarrow S_{\Delta}$ of $T_{\log }$ is trivial, and this shows that the restriction of $h$ to $S_{\Delta}$ is induced by an element $x$ of $X$. But $h$ kills $T$, and this implies that $x: T \rightarrow \mathbb{G}_{m, \log }$ is trivial, that is, $x$ is trivial. Hence the restriction of $h$ to $S_{\Delta}$ is trivial.

Proof of $\mathcal{E} x t\left(T_{\log }^{(Y)}, H\right)=0$. Let $1 \rightarrow H \rightarrow F \stackrel{p}{\rightarrow} T_{\log }^{(Y)} \rightarrow 1$ be an exact sequence. We show that this sequence has a splitting $s: T_{\log }^{(Y)} \rightarrow F$ locally on $S$. For a finitely generated subcone $\Delta$ of $C$, consider the $H$-torsor $F_{\Delta}:=p^{-1}\left(S_{\Delta}\right)$ over $S_{\Delta}$. By the generality of sites, for any sheaf of abelian 
groups $\mathcal{F}$ on $\left(\mathrm{fs} / S_{\Delta}\right)$ and any $q \in \mathbb{Z}$, the cohomology group $H^{q}\left(\left(\mathrm{fs} / S_{\Delta}\right), \mathcal{F}\right)$ coincides with the $H^{q}$ of the restriction of $\mathcal{F}$ to the small étale site $S_{\text {ét }}$ of $S$. By applying this to $\mathcal{F}=H$ and $q=0,1$, we see that the category of $H$-torsors on (fs $/ S_{\Delta}$ ) is equivalent to that of $H$-torsors on $S_{\text {ét }}$. Since the unit section $S \rightarrow S_{\Delta}$ of $T_{\log }^{(Y)}$ induces the identity functor of the small étale site of $S$, the pull back by this morphism gives an equivalence from the category of $H$-torsors on ( $\left.\mathrm{fs} / S_{\Delta}\right)$ to the category of $H$-torsors on $(\mathrm{fs} / S)$. Since the pull back of $F_{\Delta}$ by the unit section $S \rightarrow S_{\Delta}$ of $T_{\log }^{(Y)}$ is the trivial $H$-torsor $H \subset F$, the above equivalence shows that there is a unique section $s_{\Delta}: S_{\Delta} \rightarrow F_{\Delta}$ of $p: F_{\Delta} \rightarrow S_{\Delta}$ whose pull back by the unit section $S \rightarrow S_{\Delta}$ of $T_{\log }^{(Y)}$ coincides with the unit section of $H$. Since these $s_{\Delta}$ are compatible with the inclusion morphisms $S_{\Delta} \rightarrow S_{\Delta^{\prime}}$ for $\Delta \subset \Delta^{\prime}$, and since $T_{\log }^{(Y)}$ is the union of $S_{\Delta}$ for all $\Delta$, we have a section $s: T_{\log }^{(Y)} \rightarrow F$ of $p: F \rightarrow T_{\log }^{(Y)}$ by gluing $s_{\Delta}$. It is easily checked that $s$ is a homomorphism.

Proof of $\mathcal{E} x t\left(T_{\log }^{(Y)}, \mathbb{G}_{m, \log }\right)=0$. Let $1 \rightarrow \mathbb{G}_{m, \log } \rightarrow F \stackrel{p}{\rightarrow} T_{\log }^{(Y)} \rightarrow 1$ be an exact sequence. We show that this sequence has a splitting $s: T_{\log }^{(Y)} \rightarrow F$ locally on $S$. For a finitely generated subcone $\Delta$ of $C$, consider the $\mathbb{G}_{m, \log ^{-}}$ torsor $F_{\Delta}:=p^{-1}\left(S_{\Delta}\right)$ over $S_{\Delta}$. The set of isomorphism classes of all $\mathbb{G}_{m, \log ^{-}}$ torsors on $\left(\mathrm{fs}_{\mathrm{s}} / S_{\Delta}\right)$ is identified with $H^{1}\left(S_{\text {ét }}, M_{S_{\Delta}}^{\mathrm{gp}}\right)$. If $\Delta$ is sufficiently large, by $7.9(2), M_{S_{\Delta}}^{\mathrm{gp}}=M_{S}^{\mathrm{gp}} \times X$ and this sheaf on $S_{\text {ét }}$ is independent of the choice of such $\Delta$. Thus the category of $\mathbb{G}_{m, \log }$-torsors on ( $\left.\mathrm{fs} / S_{\Delta}\right)$ is independent of the choice of such $\Delta$. Hence by working locally on $S$, we may assume that there is a section $s_{\Delta}: S_{\Delta} \rightarrow F_{\Delta}$ for all $\Delta$ which are compatible with the inclusion maps between $S_{\Delta}$. Let $s: T_{\mathrm{log}}^{(Y)} \rightarrow F$ be the unique morphism whose restriction to $S_{\Delta}$ coincides with $s_{\Delta}$ for all $\Delta$. The problem is that $s$ need not be a homomorphism. Consider the morphism

$$
g: T_{\log }^{(Y)} \times T_{\log }^{(Y)} \longrightarrow \mathbb{G}_{m, \log } ; g(a, b)=s(a+b) s(a)^{-1} s(b)^{-1} .
$$

Then $g$ satisfies

$$
g(a, b) \cdot g(a, b+c)^{-1} \cdot g(a+b, c) \cdot g(b, c)^{-1}=1 .
$$

Since $\operatorname{Mor}\left(S_{\Delta} \times{ }_{S} S_{\Delta}, \mathbb{G}_{m, \log }\right)=\Gamma\left(S, M_{S}^{\mathrm{gp}} \times X \times X\right)$ for any sufficiently big $\Delta$ by Proposition $7.9(2), g$ corresponds to an element $(\mu, x, y)$ of $\Gamma\left(S, M_{S}^{\mathrm{gp}} \times\right.$ $X \times X)$. The property $(*)$ of $g$ implies

$$
\begin{array}{r}
(\mu, x, y, 1) \cdot(\mu, x, y, y)^{-1} \cdot(\mu, x, x, y) \cdot(\mu, 1, x, y)^{-1}=1 \\
\text { in } \Gamma\left(S, M_{S}^{\mathrm{gp}} \times X \times X \times X\right) .
\end{array}
$$


This implies $x=y=1$, and hence $g$ is the constant morphism with value $\mu$. Hence if we replace $s$ by $\mu s$, then $g$ becomes trivial and hence $s$ becomes a homomorphism.

7.18. We prove (1) of Theorem 7.3.

By the exact sequence $0 \rightarrow T_{\log }^{(Y)} \rightarrow G_{\log }^{(Y)} \rightarrow B \rightarrow 0$, the results on $\mathcal{H o m}\left(G_{\log }^{(Y)}, H\right)$ and $\mathcal{E} x t\left(G_{\log }^{(Y)}, H\right)$ follow from $\mathcal{H o m}\left(T_{\log }^{(Y)}, H\right)=0$ and $\mathcal{E} x t\left(T_{\log }^{(Y)}, H\right)=0(7.17)$.

From the exact sequence $0 \rightarrow T \rightarrow T_{\log }^{(Y)} \rightarrow G_{\log }^{(Y)} / G \rightarrow 0$, we obtain an exact sequence

$$
\begin{aligned}
0 \longrightarrow \mathcal{H o m}\left(G_{\log }^{(Y)} / G, H\right) \longrightarrow \mathcal{H o m}\left(T_{\log }^{(Y)}, H\right) & \longrightarrow \mathcal{H o m}(T, H) \longrightarrow \mathcal{E} x t\left(G_{\log }^{(Y)} / G, H\right) \longrightarrow \mathcal{E} x t\left(T_{\log }^{(Y)}, H\right) .
\end{aligned}
$$

Since $\mathcal{H o m}\left(T_{\log }^{(Y)}, H\right)$ and $\mathcal{E} x t\left(T_{\log }^{(Y)}, H\right)$ vanish by 7.17 , we obtain the results on $\mathcal{H o m}\left(G_{\log }^{(Y)} / G, H\right)$ and $\mathcal{E} x t\left(G_{\log }^{(Y)} / G, H\right)$.

7.19. We prove the first part of Theorem $7.3(3)$. Let $h: G_{\log }^{(Y)} \rightarrow G_{\log }^{\prime}$ be a homomorphism. Since $\mathcal{H o m}\left(G, G_{\log }^{\prime} / G^{\prime}\right)=0$ as we have seen in Corollary $6.1 .2, h$ sends $G$ into $G^{\prime}$. This homomorphism $G \rightarrow G^{\prime}$ induces by functoriality a homomorphism $G_{\log } \rightarrow G_{\log }^{\prime}$. Let $g$ be the restriction of the last homomorphism to $G_{\log }^{(Y)}$. We prove $h=g$. In fact, $h-g$ is a homomorphism $G_{\log }^{(Y)} / G \rightarrow G_{\log }^{\prime}$ and $G_{\log }^{(Y)} / G \cong T_{\log }^{(Y)} / T$. Hence we are reduced to showing $\mathcal{H o m}\left(T_{\log }^{(Y)} / T, G_{\log }^{\prime}\right)=0$. This follows from $\mathcal{H o m}\left(T_{\log }^{(Y)} / T, B^{\prime}\right)=0$ and $\mathcal{H o m}\left(T_{\log }^{(Y)} / T, T_{\log }^{\prime}\right)=0$ proved in 7.17 .

7.20. We prove (2) of Theorem 7.3. By 7.17 and by the fact $\mathcal{H o m}\left(T_{\log }^{(Y)}, \mathbb{G}_{m, \log }\right) \cong X$ which is a special case $\left(B=0\right.$ and $\left.G^{\prime}=\mathbb{G}_{m}\right)$ of the first part of (3) of Theorem 7.3, it remains to prove $\mathcal{E} x t\left(T_{\log }^{(Y)} / T, \mathbb{G}_{m, \log }\right)=0$.

We have an exact sequence $\mathcal{H o m}\left(T_{\log }^{(Y)}, \mathbb{G}_{m, \log }\right) \rightarrow \mathcal{H o m}\left(T, \mathbb{G}_{m, \log }\right) \rightarrow$ $\mathcal{E} x t\left(T_{\log }^{(Y)} / T, \mathbb{G}_{m, \log }\right) \rightarrow \mathcal{E} x t\left(T_{\log }^{(Y)}, \mathbb{G}_{m, \log }\right)$, which can be rewritten as

$$
X \stackrel{1}{\longrightarrow} X \longrightarrow \mathcal{E} x t\left(T_{\log }^{(Y)} / T, \mathbb{G}_{m, \log }\right) \longrightarrow 0,
$$

where the first $X$ appears here by the first part of (3) of Theorem 7.3, the second $X$ appears by $\mathcal{H o m}\left(T, \mathbb{G}_{m, \log } / \mathbb{G}_{m}\right)=0(6.1 .1)$, and the last 0 appears by 7.17 . 
7.21. We prove the second part of Theorem 7.3 (3).

We have an exact sequence

$$
\begin{aligned}
\mathcal{H o m}\left(G_{\log }^{(Y)} / G, T_{\log }^{\prime}\right) & \longrightarrow \mathcal{H o m}\left(G_{\log }^{(Y)} / G, G_{\log }^{\prime} / G^{\prime}\right) \\
& \longrightarrow \mathcal{E x t}\left(G_{\log }^{(Y)} / G, T^{\prime}\right) \longrightarrow \mathcal{E} x t\left(G_{\log }^{(Y)} / G, T_{\log }^{\prime}\right) .
\end{aligned}
$$

The first and the last sheaves are zero by (2) of Theorem 7.3. The third is $\mathcal{H o m}\left(T, T^{\prime}\right)=\operatorname{Hom}\left(X^{\prime}, X\right)$ by (1) of Theorem 7.3. This completes the proof of Theorem 7.3.

Before we prove Theorem 7.4, we show the following result.

Proposition 7.22. (1) $\mathcal{E} x t(B, \mathbb{Z})=0$.

(2) $\mathcal{E} x t\left(B, \mathbb{G}_{m, \log } / \mathbb{G}_{m}\right)=0$.

Proof. Let $K$ be either $\mathbb{Z}$ or $\mathbb{G}_{m, \log } / \mathbb{G}_{m}$. Let $f:(\mathrm{fs} / B) \rightarrow(\mathrm{fs} / S)$ be the morphism of sites associated to the functor $(\mathrm{fs} / S) \rightarrow(\mathrm{fs} / B) ; U \mapsto U \times{ }_{S} B$. We first prove $R^{1} f_{*} K=0$. We have to prove that the restriction of $R^{1} f_{*} K$ to the small étale site of each object $U$ of $\left(\mathrm{fs}_{\mathrm{s}} / S\right)$ is zero. By replacing $U$ by $S$, we are reduced to proving that the stalk $\left(R^{1} f_{*} K\right)_{\bar{s}}$ is zero for any $s \in S$. Since $B$ is proper over $S$, by the proper base change theorem, the canonical map $\left(R^{1} f_{*} K\right)_{\bar{s}} \rightarrow R^{1}\left(f \times_{S} \bar{s}\right)_{*}(K)$ is injective, where $f \times_{S} \bar{s}: B \times_{S} \bar{s} \rightarrow \bar{s}$. Hence it is sufficient to prove that $H^{1}\left(B_{\text {ét }}, K\right)=0$ in the case where the underlying scheme of $S$ is the Spec of a separably closed field. In this case, $K$ is a torsion-free constant sheaf. It is well known that for a Noetherian normal scheme $W$ and for a torsion-free abelian group $J$, we have $H^{1}\left(W_{\text {ét }}, J\right)=0$.

Now let $0 \rightarrow K \rightarrow F \stackrel{p}{\rightarrow} B \rightarrow 0$ be an exact sequence. We prove that locally on $S$, there is a splitting $t: B \rightarrow F$ of this sequence. By $R^{1} f_{*} K=0$, we have, locally on $S$, a morphism $t: B \rightarrow F$ such that $p \circ t$ is the identity. The problem is that $t$ need not be a homomorphism. Since $p \circ t(0)=0$, the map $t(0)$ is a morphism $S \rightarrow K$. By replacing $t$ by $t(0)^{-1} t$ (this $t(0)$ denotes the constant morphism $B \rightarrow S \stackrel{t(0)}{\rightarrow} K$ ), we have $t$ such that $t(0)$ is the unit section of $K$. We prove that such a map $t$ is a homomorphism. For such a map $t$, consider the morphism

$$
g: B \times B \longrightarrow K ;(a, b) \longmapsto t(a+b) t(a)^{-1} t(b)^{-1} .
$$

To prove that $g$ is the unit section of $K$, since the stalk $K_{\bar{s}}(s \in S)$ is isomorphic to $K(\bar{s})$, we may assume that the underlying scheme of $S$ is the 
Spec of a separably closed field. Then the restriction of $K$ on the small étale site of $B \times B$ is a constant sheaf, and the restriction of $g$ to the origin $S \rightarrow B \times B$ of $B \times B$ coincides with the unit section of $K$. Hence $g: B \times B \rightarrow K$ is the unit section.

Corollary 7.23. $\operatorname{Ext}\left(G_{\log }^{(Y)}, \mathbb{Z}\right)=0$.

Proof. This follows from Proposition 7.22 (1) and the case $H=\mathbb{Z}$ of (1) of Theorem 7.3.

7.24. We prove Theorem 7.4.

We prove $(1)$ and $\mathcal{H o m}(A, \mathbb{Z})=0$. From the exact sequence $0 \rightarrow Y \rightarrow$ $G_{\log }^{(Y)} \rightarrow A \rightarrow 0$, we obtain an exact sequence

$$
\begin{aligned}
& 0 \longrightarrow \mathcal{H o m}(A, \mathbb{Z}) \longrightarrow \\
& \longrightarrow \mathcal{H o m}\left(G_{\log }^{(Y)}, \mathbb{Z}\right) \\
& \longrightarrow \mathcal{H o m}(Y, \mathbb{Z}) \longrightarrow \mathcal{E} x t(A, \mathbb{Z}) \longrightarrow \mathcal{E} x t\left(G_{\log }^{(Y)}, \mathbb{Z}\right)
\end{aligned}
$$

But $\mathcal{H o m}\left(G_{\log }^{(Y)}, \mathbb{Z}\right)$ vanishes by the case $H=\mathbb{Z}$ of $(1)$ of Theorem 7.3 and by $\mathcal{H o m}(B, \mathbb{Z})=0$, and $\mathcal{E} x t\left(G_{\log }^{(Y)}, \mathbb{Z}\right)$ vanishes by Corollary 7.23. This proves (1) and $\mathcal{H o m}(A, \mathbb{Z})=0$.

We prove (2), (3) and the vanishings of $\mathcal{H o m}\left(A, \mathbb{G}_{m}\right)$ and $\mathcal{H o m}(A$, $\left.\mathbb{G}_{m, \log }\right)$. By $A=\left[Y \rightarrow G_{\log }^{(Y)}\right]$ and $B=G_{\log }^{(Y)} / T_{\log }^{(Y)}$, we have an exact sequence for any sheaf $F$ of abelian groups on (fs $/ S$ )

$$
\begin{aligned}
0 \longrightarrow \operatorname{Ker}(\mathcal{H o m}(B, F) \longrightarrow \mathcal{H o m}(Y, F)) \longrightarrow \mathcal{H o m}(A, F) \longrightarrow \\
\quad \mathcal{H o m}\left(T_{\log }^{(Y)}, F\right) \longrightarrow \mathcal{E} x t([Y \rightarrow B], F) \longrightarrow \mathcal{E} x t(A, F) \longrightarrow \mathcal{E} x t\left(T_{\log }^{(Y)}, F\right) .
\end{aligned}
$$

Put $F=\mathbb{G}_{m}$. Then we obtain $(2)$ by $G^{*}=\mathcal{E} x t\left([Y \rightarrow B], \mathbb{G}_{m}\right)$ and 7.17, and we also obtain $\mathcal{H o m}\left(A, \mathbb{G}_{m}\right)=0$ by 7.17 and $\mathcal{H o m}\left(B, \mathbb{G}_{m}\right)=0$.

To prove (3), we first prove

$$
\mathcal{E} x t\left([Y \rightarrow B], \mathbb{G}_{m, \log }\right)=G_{\log }^{*} .
$$

By $7.22(2)$ and $\mathcal{H o m}\left(B, \mathbb{G}_{m, \log } / \mathbb{G}_{m}\right)=0$ (6.1.1), we have

$$
\mathcal{E} x t\left(B, \mathbb{G}_{m, \log }\right) \cong \mathcal{E} x t\left(B, \mathbb{G}_{m}\right) \text { and } \mathcal{H o m}\left(B, \mathbb{G}_{m, \log }\right) \cong \mathcal{H o m}\left(B, \mathbb{G}_{m}\right)=0 .
$$


Hence $\mathcal{E} x t\left([Y \rightarrow B], \mathbb{G}_{m, \log }\right)$ is the push out of the diagram

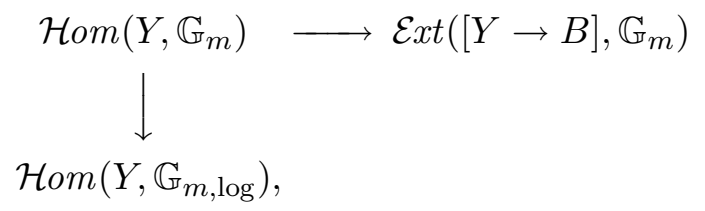

that is, the push out of $T_{\log }^{*} \leftarrow T^{*} \rightarrow G^{*}$, i.e., $G_{\log }^{*}$.

By $(*)$ and the above exact sequence for $F=\mathbb{G}_{m, \log }$, and by $\mathcal{H o m}\left(B, \mathbb{G}_{m, \log }\right)=0$, we have an exact sequence

$$
\begin{aligned}
0 \longrightarrow \mathcal{H o m}\left(A, \mathbb{G}_{m, \log }\right) \longrightarrow & \mathcal{H o m}\left(T_{\log }^{(Y)}, \mathbb{G}_{m, \log }\right) \longrightarrow G_{\log }^{*} \\
& \longrightarrow \mathcal{E} x t\left(A, \mathbb{G}_{m, \log }\right) \longrightarrow \mathcal{E} x t\left(T_{\log }^{(Y)}, \mathbb{G}_{m, \log }\right) .
\end{aligned}
$$

We have $\mathcal{H o m}\left(T_{\log }^{(Y)}, \mathbb{G}_{m, \log }\right)=X(7.3(2)), \mathcal{E} x t\left(T_{\log }^{(Y)}, \mathbb{G}_{m, \log }\right)=0(7.3(2))$, and $X \rightarrow G_{\log }^{*}$ is injective. Hence we obtain $\mathcal{E} x t\left(A, \mathbb{G}_{m, \log }\right) \cong G_{\log }^{*} / X$ and $\operatorname{Hom}\left(A, \mathbb{G}_{m, \log }\right)=0$. This completes the proof of Theorem 7.4.

7.25. Now we start the proof of Theorem 7.6.

First, we prove that the morphism $F \rightarrow \mathcal{H o m}\left(H, H^{\prime}\right)$ is an isomorphism in the case where the pairing $X \times Y \rightarrow\left(\mathbb{G}_{m, \log } / \mathbb{G}_{m}\right)_{S}$ is non-degenerate.

Let $L=\mathcal{H o m}\left(X, \mathbb{G}_{m, \log } / \mathbb{G}_{m}\right)^{(Y)}$. We have an exact sequence

$$
\mathcal{H o m}(L, \mathbb{Z}) \longrightarrow \mathcal{H o m}(Y, \mathbb{Z}) \longrightarrow \mathcal{E} x t(L / Y, \mathbb{Z}) \longrightarrow \mathcal{E} x t(L, \mathbb{Z})
$$

Applying (1) of Theorem 7.3 to $H=\mathbb{Z}$, we obtain $\mathcal{H o m}(L, \mathbb{Z})=0$ and $\mathcal{E} x t(L, \mathbb{Z}) \cong \mathcal{H o m}(T, \mathbb{Z})=0$. Hence we obtain

$$
\mathcal{E} x t(L / Y, \mathbb{Z}) \cong \operatorname{Hom}(Y, \mathbb{Z}) .
$$

Assume that we are given a homomorphism $h: L / Y \rightarrow L^{\prime} / Y^{\prime}$. Then via the above isomorphism, the homomorphism $\mathcal{E} x t\left(L^{\prime} / Y^{\prime}, \mathbb{Z}\right) \rightarrow \mathcal{E} x t(L / Y, \mathbb{Z})$ induced by $h$ gives a homomorphism $g: Y \rightarrow Y^{\prime}$. The construction of $g$ shows that the two extensions of $L / Y$ by $Y^{\prime}$, one is obtained from $0 \rightarrow$ $Y^{\prime} \rightarrow L^{\prime} \rightarrow L^{\prime} / Y^{\prime} \rightarrow 0$ by pulling back by $h$ and the other is obtained from $0 \rightarrow Y \rightarrow L \rightarrow L / Y \rightarrow 0$ by pushing forward by $g$, are isomorphic. Since $\operatorname{Hom}\left(L / Y, Y^{\prime}\right) \subset \mathcal{H o m}\left(L, Y^{\prime}\right)=0$ by the case $H=Y^{\prime}$ of (1) of Theorem 7.3, this shows that there is a unique homomorphism $f: L \rightarrow L^{\prime}$ which commutes with $g$ and $h$. By the second part of (3) of Theorem 7.3, this $f$ comes from a unique homomorphism $X^{\prime} \rightarrow X$. 
7.26. We complete the proof of Theorem 7.6.

It is sufficient to prove that for any fs $\log$ scheme $U$ over $S$ and any $u \in$ $U$, the map of stalks $F_{\bar{u}} \rightarrow \mathcal{H o m}\left(H, H^{\prime}\right)_{\bar{u}}$ is bijective. As is easily seen, the map $F_{\bar{u}} \rightarrow F(\bar{u})$ is bijective. The map $F(\bar{u}) \rightarrow \mathcal{H o m}\left(H, H^{\prime}\right)(\bar{u})$ is bijective by 7.25. Hence it is sufficient to prove that the map $\mathcal{H o m}\left(H, H^{\prime}\right)_{\bar{u}} \rightarrow$ $\mathcal{H o m}\left(H, H^{\prime}\right)(\bar{u})$ is injective.

We construct an injective morphism of sheaves $\mathcal{H o m}\left(H, H^{\prime}\right) \rightarrow$ $\mathcal{H o m}\left(X^{\prime}, \bar{X}\right)$. Since $\mathcal{H o m}\left(X^{\prime}, \bar{X}\right)_{\bar{u}} \rightarrow \mathcal{H o m}\left(X^{\prime}, \bar{X}\right)(\bar{u})$ is bijective as is easily seen, this will prove the injectivity of $\mathcal{H o m}\left(H, H^{\prime}\right)_{\bar{u}} \rightarrow \mathcal{H o m}\left(H, H^{\prime}\right)(\bar{u})$. (The morphism which we construct is a part of the inverse map of $F \rightarrow$ $\mathcal{H o m}\left(H, H^{\prime}\right)$, but we will not need this fact.)

Working locally on $S$, take an fs chart $\mathcal{S} \rightarrow M_{S}$ and an admissible pairing $X \times Y \rightarrow \mathcal{S}^{\mathrm{gp}}$ which induces $\langle$,$\rangle .$

The pull backs by the canonical morphisms $V(\Delta) \rightarrow H$ for finitely generated subcones $\Delta$ of $C$ define an injective morphism of sheaves

$$
\operatorname{Mor}\left(H, H^{\prime}\right) \stackrel{\subset}{\longrightarrow} \underset{\Delta}{\lim } \operatorname{Mor}\left(V(\Delta), H^{\prime}\right)
$$

Here Mor denotes the sheaf of morphisms (the group structures are neglected). Let $\Delta$ be a finitely generated subcone of $C$ such that $V(\Delta)$ contains the unit section of $\mathcal{H o m}\left(X, \mathbb{G}_{m}, \log \right)^{(Y)}$, and take a finitely generated subcone $\Delta^{\prime}$ of $C$ containing $\Delta$ satisfying the conditions (i) and (ii) of Proposition 7.9 (1). Let $f: V(\Delta) \rightarrow V\left(\Delta^{\prime}\right)$ be the inclusion map, and let $H_{\Delta^{\prime}}^{\prime}$ be the restriction of $H^{\prime}$ to the small étale site of $V\left(\Delta^{\prime}\right)$. By Proposition 7.9 (2), we have

$$
f^{-1}\left(H_{\Delta^{\prime}}^{\prime}\right) \subset \mathcal{H o m}\left(X^{\prime}, M_{S}^{\mathrm{gp}} / \mathcal{O}_{S}^{\times}\right) / \bar{Y}^{\prime} \times \mathcal{H} \operatorname{Hom}\left(X^{\prime}, \bar{X}\right) .
$$

Here we denote the inverse image of the sheaf $M_{S}^{\mathrm{gp}} / \mathcal{O}_{S}^{\times}$under $V(\Delta) \rightarrow S$ by $M_{S}^{\mathrm{gp}} / \mathcal{O}_{S}^{\times}$. By the following 7.26.1 which is proved easily, and which we apply to $P=V(\Delta)$ and $Q=S, H^{0}(V(\Delta)$, ) of the sheaf on the right hand side of (1) is $H^{0}\left(S, \mathcal{H o m}\left(X^{\prime}, M_{S}^{\mathrm{gp}} / \mathcal{O}_{S}^{\times}\right) / \bar{Y}^{\prime} \times \mathcal{H}\right.$ om $\left.\left(X^{\prime}, \bar{X}\right)\right)$. Hence the inclusions (1) for varying $\Delta$ give an injective morphism

$$
\lim _{\Delta} \operatorname{Mor}\left(V(\Delta), H^{\prime}\right) \stackrel{\subset}{\longrightarrow} \mathcal{H o m}\left(X^{\prime}, \mathbb{G}_{m, \log } / \mathbb{G}_{m}\right) / \bar{Y}^{\prime} \times \mathcal{H} o m\left(X^{\prime}, \bar{X}\right) .
$$

For $g \in \mathcal{M o r}\left(H, H^{\prime}\right)$, if we denote the image of $g$ in $\mathcal{H o m}\left(X^{\prime}, \mathbb{G}_{m, \log } / \mathbb{G}_{m}\right) / \bar{Y}^{\prime}$ $\times \mathcal{H o m}\left(X^{\prime}, \bar{X}\right)$ as $(c, h)$ with $c \in \mathcal{H}$ om $\left(X^{\prime}, \mathbb{G}_{m, \log } / \mathbb{G}_{m}\right) / \bar{Y}^{\prime}$ and $h: X^{\prime} \rightarrow \bar{X}$, 
then the composition $g \circ 0: S \rightarrow H^{\prime}$ of $g$ with the unit section $S \rightarrow H$ coincides with $c$. Hence if $g$ is a homomorphism, then $c$ vanishes. Hence we have an injective morphism of sheaves $\mathcal{H o m}\left(H, H^{\prime}\right) \rightarrow \mathcal{H o m}\left(X^{\prime}, \bar{X}\right)$.

7.26.1. Let $a: P \rightarrow Q, b: Q \rightarrow P$ be continuous maps of topological spaces such that $a \circ b$ is the identity map of $Q$ and such that $a^{-1}(s)$ is connected for any $s \in Q$. Then for any sheaf $F$ on $Q$, the canonical map $F \rightarrow a_{*} a^{-1}(F)$ is an isomorphism.

\section{$\S 8$. Proofs of Section 3}

We prove Theorem 3.4. Since a pointwise polarizable log 1-motif of type $(X, Y)$ induces a non-degenerate admissible pairing $X \times Y \rightarrow\left(\mathbb{G}_{m, \log } / \mathbb{G}_{m}\right)_{S}$, $(7.5(1))$, it is sufficient to prove the following theorem.

TheOREm 8.1. Let $\mathcal{C}_{1}$ be the category of log 1-motifs $\left[Y \rightarrow G_{\log }\right]$ such that the induced pairing $X \times Y \rightarrow\left(\mathbb{G}_{m, \log } / \mathbb{G}_{m}\right)_{S}$ is admissible and nondegenerate. Let $\mathcal{C}_{2}$ be the category of sheaves of abelian groups on $(\mathrm{fs} / S)$. Then the functor

$$
\mathcal{C}_{1} \longrightarrow \mathcal{C}_{2}:\left[Y \rightarrow G_{\log }\right] \longmapsto G_{\log }^{(Y)} / Y
$$

is fully faithful.

8.2. Let $M=\left[Y \rightarrow G_{\log }\right]$ and $M^{\prime}=\left[Y^{\prime} \rightarrow G^{\prime} \log \right]$ be objects of $\mathcal{C}_{1}$ and let $A=G_{\log }^{(Y)} / Y, A^{\prime}=G_{\log }^{\left(Y^{\prime}\right)} / Y^{\prime} \in \mathcal{C}_{2}$.

We show that a morphism $h: A \rightarrow A^{\prime}$ comes from a unique homomorphism $M \rightarrow M^{\prime}$.

First, by Theorem 7.4 (1), we have

$$
\mathcal{E} x t(A, \mathbb{Z}) \cong \mathcal{H o m}(Y, \mathbb{Z}) .
$$

By this and by $\mathcal{H o m}(A, \mathbb{Z})=0(7.4(4))$, we see that the exact sequence $0 \rightarrow Y \rightarrow G_{\log }^{(Y)} \rightarrow A \rightarrow 0$ given by the above isomorphism is the "universal" extension of $A$ by locally constant sheaves of finitely generated free $\mathbb{Z}$-modules, that is, the initial object among them.

By the isomorphism (1), the homomorphism $\mathcal{E} x t\left(A^{\prime}, \mathbb{Z}\right) \rightarrow \mathcal{E} x t(A, \mathbb{Z})$ induced by $h$ gives a homomorphism $g: Y \rightarrow Y^{\prime}$. The construction of $g$ shows that the two extensions of $A$ by $Y^{\prime}$, one is obtained from $0 \rightarrow$ $Y^{\prime} \rightarrow G^{\prime}\left(Y^{\prime}\right) \rightarrow A^{\prime} \rightarrow 0$ by pulling back by $h$ and the other is obtained from $0 \rightarrow Y \rightarrow G_{\log }^{(Y)} \rightarrow A \rightarrow 0$ by pushing forward by $g$, are isomorphic. 
Since $\operatorname{Hom}\left(A, Y^{\prime}\right)=0$, this shows that there is a unique homomorphism $f: G_{\log }^{(Y)} \rightarrow G^{\prime\left(Y^{\prime}\right)}$ which commutes with $g$ and $h$. By the first part of (3) of Theorem 7.3, this $f$ comes from a unique homomorphism $G \rightarrow G^{\prime}$. This completes the proof of the theorem.

\section{§9. Proofs of Section 4, I}

In this section we give the proofs of results in Section 4, except that the proof of Theorem 4.10 will be given in Sections 10-11.

9.1. Proof of the equivalence of definitions stated in 4.3 .

By 7.1.2, which is applied to the pairings $\bar{X}_{\bar{s}} \times \bar{Y}_{\bar{s}} \rightarrow\left(\mathbb{G}_{m, \log } / \mathbb{G}_{m}\right)_{S, \bar{s}}$ $(s \in S)$, if the condition 4.1.2 is satisfied, then the alternative condition by using admissibility is satisfied.

We prove the converse. Assume that the conditions in the alternative definition using admissibility on $X \times Y \rightarrow\left(\mathbb{G}_{m, \log } / \mathbb{G}_{m}\right)_{S}$ are satisfied. Let $s \in S$. Then the pull back of $A$ to $($ fs $/ \bar{s})$ is associated to a polarizable $\log$ 1-motif $\left[Y^{\prime} \rightarrow G_{\log }\right]$ of type $\left(X^{\prime}, Y^{\prime}\right)$. By Theorem 7.6, the pairing $X^{\prime} \times Y^{\prime} \rightarrow\left(\mathbb{G}_{m, \log } / \mathbb{G}_{m}\right)_{\bar{s}}$ is identified with $\bar{X}_{\bar{s}} \times \bar{Y}_{\bar{s}} \rightarrow\left(\mathbb{G}_{m, \log } / \mathbb{G}_{m}\right)_{\bar{s}}$. Hence a polarization $Y^{\prime} \rightarrow X^{\prime}$ induces a homomorphism $\bar{Y}_{\bar{s}} \rightarrow \bar{X}_{\bar{s}}$ satisfying the condition (i) in the condition 4.1.2.

9.2. We explain the facts stated in 4.4. The semi-abelian part $G$ is uniquely determined by $A$ as the biggest subgroup sheaf of $A$ which is represented by a commutative group scheme over the underlying scheme of $S$ with connected fibers (endowed with the inverse image of the log structure of $S$ ). To see this, it is sufficient to prove that for any commutative group scheme $H$ over the underlying scheme of $S$ with connected fibers, we have $\operatorname{Hom}\left(H, \mathcal{H o m}\left(X, \mathbb{G}_{m, \log } / \mathbb{G}_{m}\right)^{(Y)} / \bar{Y}\right)=0$. This last fact is shown by the same method as Lemma 6.1.1 just replacing $\mathbb{G}_{m, \log } / \mathbb{G}_{m}$ in the proof of it by $\mathcal{H o m}\left(X, \mathbb{G}_{m, \log } / \mathbb{G}_{m}\right)^{(Y)} / \bar{Y}$.

9.3. Since $G$ is uniquely determined by $A$, the quotient $A / G=$ $\operatorname{Hom}\left(X, \mathbb{G}_{m, \log } / \mathbb{G}_{m}\right)^{(Y)} / \bar{Y}$ is uniquely determined by $A$. By Theorem 7.6 , this implies that the sheaves $\bar{X}$ and $\bar{Y}$ and the pairing $\bar{X} \times \bar{Y} \rightarrow\left(\mathbb{G}_{m, \log } / \mathbb{G}_{m}\right)_{S}$ are uniquely determined by $A$.

By the uniqueness, they exist globally on $S$ (local objects glue together into a global object).

Remark 9.3.1. The sheaf $\bar{X}$ in fact coincides with the "character sheaf" of the semi-abelian scheme $G$ defined in [2]. We will discuss this in a sequel of this Part II. 
9.4. Proof of Proposition 4.5. We prove that a log abelian variety with constant degeneration is a log abelian variety. It is sufficient to prove that the condition 4.1.3 is satisfied.

Let $U$ be an fs $\log$ scheme over $S$, let $f: U \rightarrow A$ be a morphism, let $0: U \rightarrow A$ be the unit section, and let $E(f, 0)$ be the equalizer of $f$ and 0 defined as a subsheaf of $U$ over (fs $/ S$ ). It is sufficient to show that $E(f, 0)$ is represented by an fs $\log$ scheme over $U$ whose underlying scheme is finite over that of $U$. It is enough to show it étale locally on $S$. Hence we may assume that $X$ and $Y$ are constant and that there are a chart $\mathcal{S} \rightarrow M_{S}$ with an fs monoid $\mathcal{S}$ and an admissible pairing $X \times Y \rightarrow \mathcal{S}^{\mathrm{gp}}$ which induce the given pairing $X \times Y \rightarrow \mathbb{G}_{m, \log } / \mathbb{G}_{m}$. Since $A=G_{\log }^{(Y)} / Y$, étale locally on $U$, the morphism $f$ lifts to $f^{\prime}: U \rightarrow G_{\log }^{(Y)}$. Then $E(f, 0)$ is the disjoint union of subsheaves which are isomorphic to $E\left(f^{\prime}+y, 0\right)$ for $y \in Y$. Since $G_{\log }^{(Y)}=\bigcup_{\Delta} G_{\log }^{(\Delta)}$, where $\Delta$ ranges over all finitely generated subcones of $C$ and $G_{\log }^{(\Delta)}$ denotes the inverse image of $\bar{V}(\Delta)$ under $G_{\log } \rightarrow$ $\mathcal{H o m}\left(X, \mathbb{G}_{m, \log } / \mathbb{G}_{m}\right)$, étale locally on $U, f^{\prime}+y$ is a morphism into $G_{\log }^{(\Delta)}$ for some $\Delta$. We claim that $\bar{V}(\Delta) \cap \bar{V}(\Delta+y)=\varnothing$ for almost all $y \in Y$ and this shows that $G_{\log }^{(\Delta)} \cap G_{\log }^{(\Delta+y)}=\varnothing$ for almost all $y \in Y$.

We prove the claim. When $\sigma$ ranges over all faces of $\mathcal{S}$, as a set, $\operatorname{Spec}(\mathbb{Z}[\mathcal{S}])$ is a disjoint union of the locally closed subschemes $Z(\sigma)=$ $\operatorname{Spec} \mathbb{Z}\left[\sigma^{\mathrm{gp}}\right]=\operatorname{Spec}\left(\mathbb{Z}\left[\mathcal{S} \sigma^{\mathrm{gp}}\right] /\left(\mathcal{S} \sigma^{\mathrm{gp}}-\sigma^{\mathrm{gp}}\right)\right)$. It suffices to show that for each $\sigma$ such that $S \times \times_{\operatorname{Spec} \mathbb{Z}[\mathcal{S}]} Z(\sigma)$ is not empty, $V(\Delta \cap(\Delta+y)) \times_{\operatorname{Spec} \mathbb{Z}[\mathcal{S}]} Z(\sigma)$ is empty for almost all $y$, or equivalently that the image of $\Delta \cap(\Delta+y)$ in $\operatorname{Hom}(\mathcal{S}, \mathbb{N})$ does not intersect with the set $\{N \in \operatorname{Hom}(\mathcal{S}, \mathbb{N}) \mid \operatorname{Ker}(N)=\sigma\}$ for almost all $y$. By Lemma $7.12(2)$, we may assume that $\Delta=C(a)$ for some $a$. Then by [6] 5.2.7, there are only a finite number of $y$ modulo $Y_{\sigma}$ that fail the condition. Finally, since $S \times{ }_{\operatorname{Spec} \mathbb{Z}[\mathcal{S}]} Z(\sigma)$ is not empty, $X \times Y \rightarrow \mathcal{S}^{\mathrm{gp}} / \sigma^{\mathrm{gp}}$ is non-degenerate. This implies $Y_{\sigma}=\{1\}$, and completes the proof of the claim.

Hence it is sufficient to prove that for each $f: U \rightarrow G_{\text {log }}^{(\Delta)}$, the equalizer $E(f, 0)$ is represented by an fs log scheme over $U$ whose underlying scheme is finite over that of $U$. But this $E(f, 0) \rightarrow U$ is the pull back of the diagonal $G_{\log }^{(\Delta)} \rightarrow G_{\log }^{(\Delta)} \times_{S} G_{\log }^{(\Delta)}$ by $(f, 0): U \rightarrow G_{\log }^{(\Delta)} \times_{S} G_{\log }^{(\Delta)}$ (the fiber products are those in the category of fs $\log$ schemes), and $G_{\log }^{(\Delta)}$ is separated over $S$ since it is a scheme which is affine over $B$. Hence $E(f, 0) \rightarrow U$ is represented by a finite morphism. 
9.5. Proof of Theorem 4.6 (a criterion which tells when a log abelian variety is with constant degeneration).

We prove (1). The only if part is clear. We prove the if part. We may assume that the condition in 4.1.2 is satisfied not only étale locally but globally on $S$. Assume that the rank of the torus part of the fiber of $G$ is locally constant. Then since the rank of the stalk of $\bar{X}$ at $\bar{s}$ is the rank of the torus part of the fiber of $G$ on $s \in S$, we see that $\bar{X}$ is locally constant. Since the rank of $\bar{Y}$ at $\bar{s}$ is equal to the rank of $\bar{X}$ at $\bar{s}$, we see that $\bar{Y}$ is also locally constant. Hence we may assume that the admissible pairing $X \times Y \rightarrow\left(\mathbb{G}_{m, \log } / \mathbb{G}_{m}\right)_{S}$ which appears in the second condition 4.1.2 in the definition of log abelian variety is non-degenerate (i.e. $X=\bar{X}$ and $Y=\bar{Y}$ ).

Hence we can apply Theorem 7.3.

Let $L=\mathcal{H o m}\left(X, \mathbb{G}_{m, \log } / \mathbb{G}_{m}\right)^{(Y)}$. Let

$$
0 \longrightarrow G \longrightarrow \widetilde{A} \longrightarrow L \longrightarrow 0
$$

be the exact sequence obtained from $0 \rightarrow G \rightarrow A \rightarrow L / Y \rightarrow 0$ by pulling back by $L \rightarrow L / Y$. By (1) of Theorem 7.3 which we apply by taking the present $G$ as $H$, we have that this extension $0 \rightarrow G \rightarrow \widetilde{A} \rightarrow L \rightarrow 0$ comes from the canonical extension $0 \rightarrow T \rightarrow T_{\log }^{(Y)} \rightarrow L \rightarrow 0$ by pushing forward by a homomorphism $T \rightarrow G$. By checking at a fiber, we see that at each fiber, this homomorphism $T \rightarrow G$ is an embedding whose quotient is an abelian variety. On the other hand, by [2] I, 2.11, the assumption on the locally constant torus rank shows that $G$ is an extension of an abelian scheme by a torus. Hence this homomorphism $T \rightarrow G$ is an isomorphism between $T$ and the torus part of $G$. This shows that the extension (1) of $L$ by $G$ is isomorphic to the standard extension

$$
0 \longrightarrow G \longrightarrow G_{\log }^{(Y)} \longrightarrow L \longrightarrow 0
$$

$\left(G_{\log }^{(Y)}\right.$ denotes the inverse image of $L$ in $\left.G_{\log }\right)$. We have $Y \rightarrow G_{\log }$ as the composite of $Y \rightarrow \widetilde{A} \cong G_{\log }^{(Y)}$. We have thus $A=G_{\log }^{(Y)} / Y$. Since this $\log$ 1-motif $\left[Y \rightarrow G_{\log }\right]$ is pointwise polarizable by Theorem 8.1, we see that $A$ is a $\log$ abelian variety with constant degeneration.

We prove (2). This follows from (1) and Lemma 9.6 below.

Lemma 9.6. Let $X$ and $Y$ be finitely generated free $\mathbb{Z}$-modules, and let $X \times Y \rightarrow\left(\mathbb{G}_{m, \log } / \mathbb{G}_{m}\right)_{S}$ be an admissible pairing. Assume that $M_{S} / \mathcal{O}_{S}^{\times}$is a locally constant sheaf on the small étale site of $S$. Then $\bar{X}$ and $\bar{Y}$ are locally constant on $(\mathrm{fs} / S)$. 
The proof is immediate.

9.7. Proof of Proposition 4.7. We show that $A=\mathcal{E}_{q}$ defined in Section 1 is a $\log$ abelian variety.

By 1.7, we have that $A$ satisfies the condition 4.1.1 in the definition of $\log$ abelian variety.

The condition 4.1.2 in the definition of log abelian variety is satisfied as is seen from the exact sequence

$$
0 \longrightarrow G \longrightarrow \mathcal{E}_{q} \longrightarrow \mathbb{G}_{m, \log }^{(q)} /\left(\mathbb{G}_{m} q^{\mathbb{Z}}\right) \longrightarrow 0
$$

which follows from 5.3-5.4.

We prove that the condition 4.1.3 is satisfied. Morphisms $f, g: U \rightarrow A$ locally come from $f^{\prime}, g^{\prime}: \mathcal{E}_{q^{n}}^{(I)}$ for some $n, I$. By the exact sequence $0 \rightarrow$ $\mathbb{Z} / n \mathbb{Z} \rightarrow \mathcal{E}_{q^{n}} \rightarrow \mathcal{E}_{q} \rightarrow 0$, we see that the equalizer $E(f, g)$ is the disjoint union of $E\left(f^{\prime}, g^{\prime} q^{i}\right)$ for $i \in \mathbb{Z} / n \mathbb{Z}$. It is hence sufficient to prove that for any morphisms $f, g: U \rightarrow \mathcal{E}_{q^{n}}^{(I)}$ over $S$, the equalizer $E(f, g)$ is finite over $U$. But this is valid since $\mathcal{E}_{q^{n}}^{(I)}$ is a separated scheme over $O_{K}$.

9.8. We start the proof of the proposition 4.8 about global log abelian varieties. We first show that if $U$ is an fs $\log$ scheme over $S$, the diagram

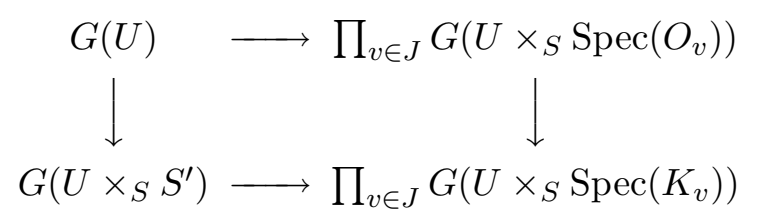

is cartesian. By limit argument, this is reduced to the case where $U$ is Noetherian, and to the following proposition.

Proposition 9.9. Let $R$ be a Noetherian commutative ring, $f$ an element of $R$, and let $R^{\prime}$ be a commutative ring over $R$ which is flat over $R$ such that $R / f R \stackrel{\simeq}{\rightarrow} R^{\prime} / f R^{\prime}$. Then for a separated scheme $F$ over $R$, the following diagram is cartesian.

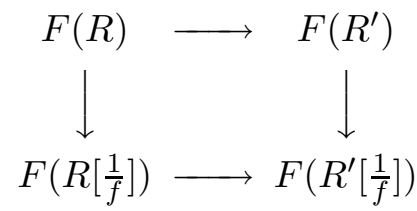


Proof. Let

$$
I=\left\{a \in R \mid f^{n} a=0 \text { for some } n \geq 1\right\} .
$$

It is sufficient to prove that the following two squares are cartesian.

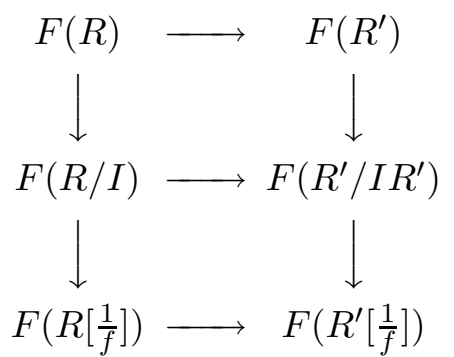

We first prove that the upper square is cartesian. First note that $R / f^{n} R \stackrel{\simeq}{\rightrightarrows}$ $R^{\prime} / f^{n} R^{\prime}$ for any $n \geq 1$ (this is deduced from $R / f R \stackrel{\simeq}{\rightarrow} R^{\prime} / f R^{\prime}$ by induction on $n$ and by the flatness of $R \rightarrow R^{\prime}$ ). Since $R$ is Noetherian, there is an $n \geq 1$ such that $f^{n} I=0$. We have $f^{n} R \cap I=0$ as is easily seen. Hence we have a cartesian diagram of surjections of rings

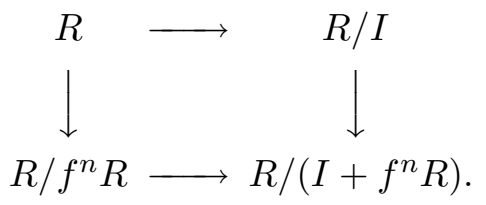

Hence the diagram

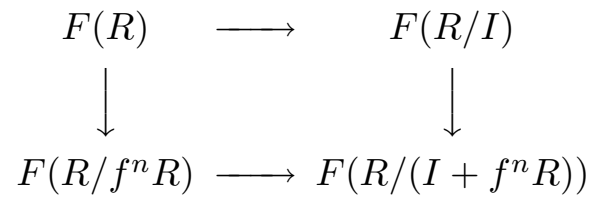

is cartesian. The image of $f$ in $R / I$ is a non zerodivisor. By the flatness of $R \rightarrow R^{\prime}$, the image of $f$ in $R^{\prime} / I R^{\prime}$ is a non zerodivisor, and it follows that $I R^{\prime}=\left\{a \in R^{\prime} \mid f^{n} a=0\right.$ for some $\left.n \geq 1\right\}$. Hence we have again a cartesian diagram if we replace $R$ in the diagram (2) by $R^{\prime}$, and $I$ by $I R^{\prime}$. Since $R / f^{n} R \stackrel{\simeq}{\rightarrow} R^{\prime} / f^{n} R^{\prime}$, this diagram is written as

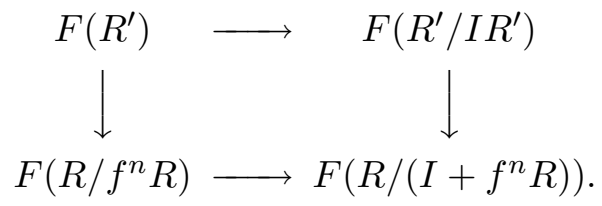


By comparing the two diagrams (2) and (3), we see that the upper square of (1) is cartesian.

We next prove that the lower square in (1) is cartesian. For this, we may assume that $f$ is a non zerodivisor in $R$ and $I=0$. By the fpqc descent for the faithfully flat morphism $R \rightarrow R[1 / f] \times R^{\prime}$, we have

$$
F(R) \stackrel{\simeq}{\longrightarrow}\left\{(a, b) \in F\left(R\left[\frac{1}{f}\right]\right) \times F\left(R^{\prime}\right) \mid g(a)=h(b), p_{1}(b)=p_{2}(b)\right\},
$$

where $g: F\left(R\left[\frac{1}{f}\right]\right) \rightarrow F\left(R^{\prime}\left[\frac{1}{f}\right]\right), h: F\left(R^{\prime}\right) \rightarrow F\left(R^{\prime}\left[\frac{1}{f}\right]\right), p_{1}, p_{2}: F\left(R^{\prime}\right) \rightarrow$ $F\left(R^{\prime} \otimes_{R} R^{\prime}\right)$. Since the image of $f$ in $R^{\prime} \otimes_{R} R^{\prime}$ is a non zerodivisor, $R^{\prime} \otimes_{R} R^{\prime} \rightarrow\left(R^{\prime} \otimes_{R} R^{\prime}\right)[1 / f]$ is injective. Since $F$ is separated, this shows that the map $F\left(R^{\prime} \otimes_{R} R^{\prime}\right) \rightarrow F\left(\left(R^{\prime} \otimes_{R} R^{\prime}\right)[1 / f]\right)$ is injective. Let $(a, b) \in$ $F(R[1 / f]) \times F\left(R^{\prime}\right)$ and assume $g(a)=h(b)$. Then the images of $p_{1}(b)$ and $p_{2}(b)$ in $F\left(\left(R^{\prime} \otimes_{R} R^{\prime}\right)[1 / f]\right)$ coincides with the image of $a$, and hence $p_{1}(b)=p_{2}(b)$. This shows that there is a unique element $c$ of $F(R)$ whose image in $F(R[1 / f]) \times F\left(R^{\prime}\right)$ is $(a, b)$.

9.10. Proof of Proposition 4.8. First we prove that $A$ satisfies the second condition in 4.1 of a log abelian variety by showing 4.8 (3). By comparing the cartesian diagram defining $A$ in 4.8 (1) and the cartesian diagram for $G$ in 9.8 , we have an exact sequence

$$
0 \longrightarrow G(U) \longrightarrow A(U) \longrightarrow \prod_{v \in J} \mathcal{E}_{q_{v}}\left(U \times{ }_{S} \operatorname{Spec}\left(O_{v}\right)\right) / G\left(U \times{ }_{S} \operatorname{Spec}\left(O_{v}\right)\right) .
$$

Hence we have an exact sequence

$$
0 \longrightarrow G \longrightarrow A \longrightarrow \bigoplus_{v} F^{(v)}, \quad \text { where } F^{(v)}=i_{v *}\left(\mathbb{G}_{m, \log }^{\left(q_{v}\right)} /\left(\mathbb{G}_{m} \cdot q_{v}^{\mathbb{Z}}\right)\right) \text {. }
$$

It remains to prove that the last arrow is surjective. It is sufficient to prove assuming $U$ is of finite type over $S$ that $A_{\bar{u}}=A\left(\mathcal{O}_{U, \bar{u}}\right) \rightarrow F^{(v)}\left(\mathcal{O}_{U, \bar{u}}\right)$ is surjective for any $u \in U$ lying over $v$. Let $R^{\prime}=\mathcal{O}_{U, \bar{u}}$ and let $R$ be the completion of $R^{\prime}$. We first show that $A(R) \rightarrow F^{(v)}(R)$ is surjective. For $f \in F^{(v)}(R)$, there exists an element $g \in \mathcal{E}_{q_{v}}(R)$ with image $f$. Then $(g, g) \in G\left(\operatorname{Spec}(R) \times{ }_{S} S^{\prime}\right) \times \mathcal{E}_{q_{v}}\left(\operatorname{Spec}(R) \times{ }_{S} \operatorname{Spec}\left(O_{v}\right)\right)$ defines an element $h$ of $A(R)$. Thus $A(R) \rightarrow F^{(v)}(R)$ is surjective. Via the isomorphism $F^{(v)}\left(R^{\prime}\right) \stackrel{\cong}{\rightrightarrows}$ $F^{(v)}(R)$, we regard $f$ also as an element of $F^{(v)}\left(R^{\prime}\right)$. Then there are a finitely generated subring $R^{\prime \prime}$ of $R$ over $R^{\prime}$ and an element $h^{\prime \prime} \in A\left(R^{\prime \prime}\right)$ whose image in $A(R)$ coincides with $h$ and whose image in $F^{(v)}\left(R^{\prime \prime}\right)$ coincides with the 
image of $f$ under $F^{(v)}\left(R^{\prime}\right) \rightarrow F^{(v)}\left(R^{\prime \prime}\right)$. By Artin's approximation (which holds since $S$ is excellent), there is an $R^{\prime}$-homomorphism $R^{\prime \prime} \rightarrow R^{\prime}$. Let $h^{\prime}$ be the image of $h^{\prime \prime}$ in $A\left(R^{\prime}\right)$ under $A\left(R^{\prime \prime}\right) \rightarrow A\left(R^{\prime}\right)$. Then the image of $h^{\prime}$ in $F^{(v)}\left(R^{\prime}\right)$ is $f$.

Next we prove (2) of Proposition 4.8.

The pull back of $A$ to $S^{\prime}$ is clearly $G$. We prove that the pull back $\left.A\right|_{O_{v}}$ of $A$ to $O_{v}$ is $\mathcal{E}_{q_{v}}$ for each $v \in J$. We have a canonical homomorphism $\left.A\right|_{O_{v}} \rightarrow \mathcal{E}_{q_{v}}$, and a commutative diagram of exact sequences

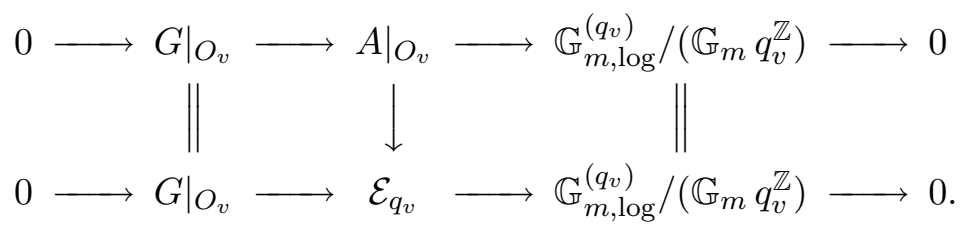

This proves $\left.A\right|_{O_{v}} \cong \mathcal{E}_{q_{v}}$.

Now by (2) of Proposition 4.8, we see that $A$ satisfies the first condition 4.1.1.

It remains to prove that $A$ satisfies the third condition 4.1.3. Let $U$ be an fs $\log$ scheme over $S$, let $f: U \rightarrow A$ be a morphism, and let $E(f, 0)$ be the equalizer of $f$ and the zero section 0 of $A$. We will prove that $E(f, 0)$ is representable étale locally on $U$. If we prove this, then since the morphism from the disjoint union of $S^{\prime}$ and $\operatorname{Spec}\left(O_{v}\right)$ for all $v \in J$ to $S$ is faithfully flat, the finiteness of $E(f, 0)$ over $U$ is reduced to the fact the zero section of $G \times{ }_{S} S^{\prime}$ is finite and the zero section of $\mathcal{E}_{q_{v}}$ is represented by finite morphisms for any $v \in J$. (Note that by the finiteness, étale local representability implies the representabiliy.)

To prove $E(f, 0)$ is étale locally representable, working locally on $S$, we may assume that $J$ is a one point set $\{v\}$ and there is an element $t$ of $\mathcal{O}(S)$ whose image in $K_{v}$ belongs to $q_{v} O_{v}^{\times}$and which is invertible on $S \backslash\{v\}$. We have $i_{v *}\left(\mathbb{G}_{m, \log }^{\left(q_{v}\right)} /\left(\mathbb{G}_{m} q_{v}^{\mathbb{Z}}\right)\right)=\mathbb{G}_{m, \log }^{(t)} /\left(\mathbb{G}_{m} t^{\mathbb{Z}}\right)$. We prove

9.10.1. For any morphism $f: U \rightarrow \mathbb{G}_{m, \log }^{(t)} /\left(\mathbb{G}_{m} t^{\mathbb{Z}}\right)$, the equalizer $E(f, 0)$ is representable étale locally on $U$. Here 0 denotes the unit section of $\mathbb{G}_{m, \log }^{(t)} /\left(\mathbb{G}_{m} t^{\mathbb{Z}}\right)$.

If we prove this, then for $f: U \rightarrow A$, the étale local representability of $E(f, 0)$ is shown as follows. Let $\bar{f}: U \rightarrow \mathbb{G}_{m, \log }^{(t)} /\left(\mathbb{G}_{m} t^{\mathbb{Z}}\right)$ be the morphism induced by $f$. Then $f$ induces $f^{\prime}: E(\bar{f}, 0) \rightarrow G$, and $E(f, 0)$ is identified 
with the equalizer of $f^{\prime}$ and $0: E(\bar{f}, 0) \rightarrow G$ which is representable over $E(\bar{f}, 0)$.

We prove 9.10.1. Étale locally on $U$, take a lifting $\tilde{f}: U \rightarrow \mathbb{G}_{m, \log } / \mathbb{G}_{m}$ of $f$. Then $E(f, 0)$ is the union of $E\left(\widetilde{f} t^{n}, 0\right)$ for $n \in \mathbb{Z}$. If $m, n \in \mathbb{Z}$ and $m \neq n$, the intersection of $E\left(\widetilde{f} t^{m}, 0\right)$ and $E\left(\widetilde{f} t^{n}, 0\right)$ is open both in $E\left(\widetilde{f} t^{m}, 0\right)$ and in $E\left(\widetilde{f} t^{n}, 0\right)$ (the open sets are the parts where $t \bmod \mathbb{G}_{m}$ vanishes), and hence it remains to prove

9.10.2. For any fs $\log$ scheme $U$ and any morphisms $f, g: U \rightarrow$ $\mathbb{G}_{m, \log } / \mathbb{G}_{m}$, the equalizer $E(f, g)$ is étale locally on $U$, representable by an fs $\log$ scheme over $U$.

In fact, étale locally on $U$, writing $f=f_{1} f_{2}^{-1}, g=g_{1} g_{2}^{-1}, E(f, g)=$ $E\left(f_{1} g_{2}, f_{2} g_{1}\right)$. Hence we may assume that $f, g$ come from sections of $M_{U}$, which are denoted by the same letters respectively. Then $E(f, g)$ is represented by the fiber product of $U \rightarrow \operatorname{Spec}\left(\mathbb{Z}\left[T_{1}, T_{2}\right]\right) \leftarrow \operatorname{Spec}\left(\mathbb{Z}\left[T_{3}, T_{4}^{ \pm}\right]\right)$, where $\operatorname{Spec}\left(\mathbb{Z}\left[T_{1}, T_{2}\right]\right)$ is endowed with the log structure associated to $\mathbb{N}^{2} \rightarrow$ $\mathbb{Z}\left[T_{1}, T_{2}\right] ;(m, n) \mapsto T_{1}^{m} T_{2}^{n}, \operatorname{Spec}\left(\mathbb{Z}\left[T_{3}, T_{4}^{ \pm}\right]\right)$is endowed with the log structure associated to $\mathbb{N} \rightarrow \mathbb{Z}\left[T_{3}, T_{4}^{ \pm}\right] ; n \mapsto T_{3}^{n}, U \rightarrow \operatorname{Spec}\left(\mathbb{Z}\left[T_{1}, T_{2}\right]\right)$ is given by $T_{1} \mapsto f, T_{2} \mapsto g$, and $\operatorname{Spec}\left(\mathbb{Z}\left[T_{3}, T_{4}^{ \pm}\right]\right) \rightarrow \operatorname{Spec}\left(\mathbb{Z}\left[T_{1}, T_{2}\right]\right)$ is given by $T_{1} \mapsto T_{3}, T_{2} \mapsto T_{3} T_{4}$

\section{$\S 10$. Computation of Hom and Ext (analytic theory)}

In this section, we study Hom sheaves and Ext sheaves as in Section 7, but in analytic sites. The aim is to prove Theorem 10.3, which will be used in Section 11. Let $S$ be an fs $\log$ analytic space. Let (fsan/S) be the category of fs log analytic spaces over $S$ which we regard as a site with the usual topology. In this section, $\mathbb{G}_{a}, \mathbb{G}_{m}, \mathbb{G}_{m, \log }, V(\Delta), \bar{V}(\Delta)$ are analytic versions considered in $[6]$.

10.1. Let $X \times Y \rightarrow\left(\mathbb{G}_{m, \log } / \mathbb{G}_{m}\right)_{S}$ be an admissible pairing, that is, a pairing such that for any $s \in S$, the induced $\mathbb{Z}$-bilinear form $X \times Y \rightarrow$ $M_{S, s}^{\mathrm{gp}} / \mathcal{O}_{S, s}^{\times}$is admissible in the sense of 7.1. (We do not assume that it is non-degenerate.) Let $T=\mathcal{H o m}\left(X, \mathbb{G}_{m}\right)$. In this section, for a commutative Lie group $G$ over the underlying analytic space of $S$ with connected fibers, we compute

$$
\mathcal{H o m}\left(T_{\log }^{(Y)}, G\right), \quad \mathcal{H o m}\left(T_{\log }^{(Y)} / T, G\right), \quad \mathcal{E} x t\left(T_{\log }^{(Y)}, G\right), \quad \mathcal{E} x t\left(T_{\log }^{(Y)} / T, G\right) .
$$

Here a Lie group over an analytic space $V$ means a smooth analytic space over $V$ which is separated over $V$ endowed with an (analytic) group structure 
over $V$. (" $G$ is separated over $V$ " means that for any Hausdorff open subset $U$ of $V$, the inverse image of $U$ in $G$ is Hausdorff.)

The result on $\mathcal{E} x t\left(T_{\log }^{(Y)} / T, G\right)$ will be used in 11.5 for the proof of Theorem 4.10 .

10.2. Let $G$ be a commutative Lie group over the underlying analytic space of $S$ with connected fibers.

The exponential map $\operatorname{Lie}(G) \rightarrow G$ is surjective. Let $\mathcal{H}_{1}(G, \mathbb{Z})$ be the kernel of it, so we have an exact sequence

$$
0 \longrightarrow \mathcal{H}_{1}(G, \mathbb{Z}) \longrightarrow \operatorname{Lie}(G) \longrightarrow G \longrightarrow 0 .
$$

This $\mathcal{H}_{1}(G, \mathbb{Z})$ is the pull back to (fsan/S) of a sheaf on the underlying topological space of $S$.

We have

$$
\mathcal{H}_{1}(G, \mathbb{Z})(-1) \cong \mathcal{H o m}\left(\mathbb{G}_{m}, G\right) .
$$

$\left((-1)\right.$ means $\otimes_{\mathbb{Z}} \mathbb{Z} \cdot(2 \pi i)^{-1}$.) Indeed, a homomorphism $\mathbb{G}_{m} \rightarrow G$ gives a homomorphism from $\mathbb{Z}(1)=\mathcal{H}_{1}\left(\mathbb{G}_{m}, \mathbb{Z}\right) \rightarrow \mathcal{H}_{1}(G, \mathbb{Z})$ and this gives $\mathcal{H o m}\left(\mathbb{G}_{m}, G\right) \rightarrow \mathcal{H}_{1}(G, \mathbb{Z})(-1)$. The converse map is given as follows. A section of $\mathcal{H}_{1}(G, \mathbb{Z})$ induces a homomorphism $\mathbb{G}_{a} \rightarrow \mathbb{G}_{a} \otimes \mathcal{H}_{1}(G, \mathbb{Z})$ which sends $\mathbb{Z}$ into $\mathcal{H}_{1}(G, \mathbb{Z})$. Hence a section of $\mathcal{H}_{1}(G, \mathbb{Z})(-1)$ induces a homomorphism from $\mathbb{G}_{a} / \mathbb{Z}(1)=\mathbb{G}_{m}$ to $\left(\mathbb{G}_{a} \otimes_{\mathbb{Z}} \mathcal{H}_{1}(G, \mathbb{Z})\right) / \mathcal{H}_{1}(G, \mathbb{Z})$, and via the canonical projection $\mathbb{G}_{a} \otimes_{\mathbb{Z}} \mathcal{H}_{1}(G, \mathbb{Z}) \rightarrow \operatorname{Lie}(G)$, a homomorphism $\mathbb{G}_{m} \rightarrow \operatorname{Lie}(G) / \mathcal{H}_{1}(G, \mathbb{Z}) \simeq G$.

The isomorphism (1) induces an isomorphism

$$
X \otimes \mathcal{H}_{1}(G, \mathbb{Z})(-1) \cong \mathcal{H o m}(T, G) .
$$

THEOREM 10.3. Let $G$ be a commutative Lie group over the underlying analytic space of $S$ with connected fibers. Then the sheaves $\mathcal{H o m}\left(T_{\log }^{(Y)} / T, G\right)$ and $\mathcal{E} x t\left(T_{\log }^{(Y)}, G\right)$ are zero, we have an isomorphism

$$
\mathcal{E} x t\left(T_{\log }^{(Y)} / T, G\right) \cong \bar{X} \otimes_{\mathbb{Z}} \mathcal{H}_{1}(G, \mathbb{Z})(-1)
$$

induced by the boundary map $X \otimes \mathcal{H}_{1}(G, \mathbb{Z})(-1) \cong \mathcal{H o m}(T, G) \rightarrow$ $\mathcal{E} x t\left(T_{\log }^{(Y)} / T, G\right)$, and we have an isomorphism

$$
\mathcal{H} \operatorname{com}\left(T_{\log }^{(Y)}, G\right) \cong \operatorname{Ker}(X \rightarrow \bar{X}) \otimes_{\mathbb{Z}} \mathcal{H}_{1}(G, \mathbb{Z})(-1) .
$$

Here $\bar{X}$ denotes the image of $X$ in $\mathcal{H o m}\left(Y, \mathbb{G}_{m, \log } / \mathbb{G}_{m}\right)$.

The rest of this section is devoted to the proof of this theorem. 
10.4. It is enough to prove Theorem 10.3 locally on $S$. Hence it may be supposed that we have a chart $\mathcal{S} \rightarrow M_{S}$ with an fs monoid and an admissible pairing $X \times Y \rightarrow \mathcal{S}^{\mathrm{gp}}$ which induce the given pairing $X \times Y \rightarrow \mathbb{G}_{m, \log } / \mathbb{G}_{m}$.

Let $C \subset \operatorname{Hom}(\mathcal{S}, \mathbb{N}) \times \operatorname{Hom}(X, \mathbb{Z})$ be as in 7.7 . Let $\Delta$ be a finitely generated subcone of $C$.

Let $\mathcal{K}$ be the complex of sheaves on $S$ whose degree $n$ part $\mathcal{K}^{n}$ is $\mathcal{H}^{0}\left(V(\Delta) \times T^{n}, \mathcal{O}\right)$ for $n \geq 0$ and is 0 for $n<0$, where $\mathcal{H}^{0}\left(V(\Delta) \times T^{n}, \quad\right)$ denotes the direct image from the site $\left(\mathrm{fsan} /\left(V(\Delta) \times T^{n}\right)\right)$ to the site $(\mathrm{fsan} / S)$, and the differential $d_{n}: \mathcal{K}^{n} \rightarrow \mathcal{K}^{n+1}$ for $n \geq 0$ is defined by

$$
\begin{aligned}
\left(d_{n} f\right)\left(v, t_{1}, \ldots, t_{n+1}\right)= & f\left(t_{1} v, t_{2}, \ldots, t_{n+1}\right) \\
& +\sum_{i=1}^{n}(-1)^{i} f\left(v, t_{1}, \ldots, t_{i-1}, t_{i} t_{i+1}, t_{i+2}, \ldots, t_{n+1}\right) \\
& +(-1)^{n+1} f\left(v, t_{1}, \ldots, t_{n}\right) .
\end{aligned}
$$

For example, in the case $n=0,\left(d_{0} f\right)(v, t)=f(t v)-f(v)$. $H^{0}$.

Let $K$ be the complex defined by replacing $\mathcal{H}^{0}$ in the definition of $\mathcal{K}$ by

These $\mathcal{K}$ and $K$ are the Cech complexes associated to the covering $V(\Delta) \rightarrow \bar{V}(\Delta)$ (note

$$
V(\Delta) \times T \simeq V(\Delta) \times \bar{V}(\Delta) V(\Delta) ;(v, t) \longmapsto(v, t v)) .
$$

Lemma 10.5. Assume that the projection $\Delta \rightarrow \operatorname{Hom}(\mathcal{S}, \mathbb{N})$ is surjective. Then the canonical map $\mathcal{O}_{S} \rightarrow \mathcal{K}$ is a quasi-isomorphism. Here $\mathcal{O}_{S}$ is the sheaf $U \mapsto \mathcal{O}(U)$ on $(\mathrm{fsan} / S)$ regarded as a complex concentrated in degree 0 .

Proof. Locally on $S, S$ is isomorphic to a closed analytic subspace of $S^{\prime}=W \times \operatorname{Spec}(\mathbb{C}[\mathcal{S}])$ an whose ideal sheaf is generated by a finite number of global sections, where $W$ is a finite product of copies of $\{z \in \mathbb{C}|| z \mid<1\}$ ( $W$ is with no $\log$ structure) and $S$ is endowed with the inverse image of the $\log$ structure of $S^{\prime}$. For such $S$, we will prove that $\mathcal{O}(S) \rightarrow K$ is a quasi-isomorphism. This will show that $\mathcal{O}_{S} \rightarrow \mathcal{K}$ is a quasi-isomorphism in general.

Let the situation $S \subset S^{\prime}$ be as above, and let $K^{\prime}$ be the " $K$ for $S^{\prime \prime}$ ". Then $K=\mathcal{O}(S) \otimes_{\mathcal{O}\left(S^{\prime}\right)} K^{\prime}$. To prove that $\mathcal{O}(S) \rightarrow K$ is a quasi-isomorphism, it is sufficient to construct homomorphisms of $\mathcal{O}\left(S^{\prime}\right)$-modules

$$
s_{n}:\left(K^{\prime}\right)^{n} \rightarrow\left(K^{\prime}\right)^{n-1} \text { for } n \geq 1, \quad s_{0}:\left(K^{\prime}\right)^{0} \rightarrow \mathcal{O}\left(S^{\prime}\right)
$$


such that $d s_{n}+s_{n+1} d$ for $n \geq 0$ (resp. $s_{0} d$ ) is the identity map of $\left(K^{\prime}\right)^{n}$ (resp. $\left.\mathcal{O}\left(S^{\prime}\right)\right)$. Here we denote by $d=d_{-1}$ the canonical map $\mathcal{O}\left(S^{\prime}\right) \rightarrow\left(K^{\prime}\right)^{0}$.

We construct $s_{n}$ as follows. We may and will assume $S^{\prime}=S=W \times$ $\operatorname{Spec}(\mathbb{C}[\mathcal{S}])_{\text {an }}$

Let $P=\Delta^{\vee}$. Note

$$
\mathcal{S}=\mathcal{S} \times\{1\} \subset P \subset \mathcal{S}^{\mathrm{gp}} \times X .
$$

The surjectivity of $\Delta \rightarrow \operatorname{Hom}(\mathcal{S}, \mathbb{N})$ shows that the homomorphism $\mathcal{S} \rightarrow P$ is exact (that is, $\mathcal{S}$ coincides with the inverse image of $P$ under $\mathcal{S}^{\mathrm{gp}} \rightarrow P^{\mathrm{gp}}$ ).

The set $H^{0}\left(V(\Delta) \times T^{n}, \mathcal{O}\right)$ is identified with the set of all formal infinite sums

$$
\sum c_{p, x_{1}, \ldots, x_{n}} p \otimes x_{1} \otimes \cdots \otimes x_{n},
$$

satisfying the following condition (i), where $p$ ranges over $P, x_{i}(1 \leq i \leq n)$ ranges over $X$, and $c_{p, x_{1}, \ldots, x_{n}} \in \mathcal{O}(W)$.

(i) For any $w \in W$ and any homomorphism $\varphi: P \times X^{n} \rightarrow \mathbb{C}^{\times}$, we have

$$
\sum\left|c_{p, x_{1}, \ldots, x_{n}}(w)\right| \cdot\left|\varphi\left(p, x_{1}, \ldots, x_{n}\right)\right|<\infty .
$$

For $p \in P$, let $\bar{p}$ be the image of $p$ in $X$. Then the map

$$
H^{0}\left(V(\Delta) \times T^{n}, \mathcal{O}\right) \longrightarrow H^{0}\left(V(\Delta) \times T^{n+1}, \mathcal{O}\right)
$$

is given by (the infinite $\mathcal{O}(W)$-linear combination of)

$$
\begin{aligned}
p \otimes x_{1} \otimes \cdots & \otimes x_{n} \longmapsto p \otimes \bar{p} \otimes x_{1} \otimes \cdots \otimes x_{n} \\
& +\sum_{i=1}^{n}(-1)^{i} p \otimes x_{1} \otimes \cdots \otimes x_{i-1} \otimes x_{i} \otimes x_{i} \otimes x_{i+1} \otimes \cdots \otimes x_{n} \\
& +(-1)^{n+1} p \otimes x_{1} \otimes \cdots \otimes x_{n} \otimes 1 .
\end{aligned}
$$

We define a homotopy $\left(s_{n}\right)_{n}$ as follows. The map

$$
s_{n}: H^{0}\left(V(\Delta) \times T^{n}, \mathcal{O}\right) \longrightarrow H^{0}\left(V(\Delta) \times T^{n-1}, \mathcal{O}\right) \quad(n \geq 1)
$$

sends $p \otimes x_{1} \otimes \cdots \otimes x_{n}$ to 0 if $x_{n} \neq 1$, and to $(-1)^{n} p \otimes x_{1} \otimes \cdots \otimes x_{n-1}$ if $x_{n}=1$. The map

$$
s_{0}: H^{0}(V(\Delta), \mathcal{O}) \longrightarrow \mathcal{O}(S)
$$

sends $p \notin \mathcal{S}$ to 0 , and $p \in \mathcal{S}$ to $p$. (To prove $d_{-1} s_{0}+s_{1} d_{0}=1$, we use the exactness of $\mathcal{S} \rightarrow P$, that is, $p \in P$ belongs to $\mathcal{S}$ if and only if $\bar{p}=1$ ). 
Corollary 10.6. Assume that the projection $\Delta \rightarrow \operatorname{Hom}(\mathcal{S}, \mathbb{N})$ is surjective.

(1) $\mathcal{H}^{0}(\bar{V}(\Delta), \mathcal{O})=\mathcal{O}_{S}$

(2) $\mathcal{H}^{1}(\bar{V}(\Delta), \mathcal{O})=0$.

Here $\mathcal{H}^{m}(\bar{V}(\Delta), \quad)$ denotes the $m$-th higher direct image functor associated to the direct image functor from the category of sheaves on the site $(\mathrm{fsan} / S) / \bar{V}(\Delta)$ of the objects of $(\mathrm{fsan} / S)$ over the sheaf $\bar{V}(\Delta)$ to the category of sheaves on $(\mathrm{fsan} / S)$.

Proof. (1) In fact, $\mathcal{H}^{0}(\bar{V}(\Delta), \mathcal{O})$ is identified with the kernel of $d_{0}$ : $\mathcal{H}^{0}(V(\Delta), \mathcal{O}) \rightarrow \mathcal{H}^{0}(V(\Delta) \times T, \mathcal{O})$.

(2) A $\mathbb{G}_{a}$-torsor on $\bar{V}(\Delta)$ is a $\mathbb{G}_{a}$-torsor on $V(\Delta)$ endowed with a descent data on $V(\Delta) \times T$. Since $V(\Delta)$ is Stein locally on $S$, a $\mathbb{G}_{a}$-torsor on $V(\Delta)$ is trivial locally on $S$. For a trivial $\mathbb{G}_{a}$-torsor on $V(\Delta)$, the descent data is regarded as an element of the kernel of $d_{1}: H^{0}(V(\Delta) \times T, \mathcal{O}) \rightarrow H^{0}(V(\Delta) \times$ $\left.T^{2}, \mathcal{O}\right)$. By Lemma 10.5, it comes locally on $S$ from $H^{0}(V(\Delta), \mathcal{O})$. This shows that a $\mathbb{G}_{a}$-torsor on $\bar{V}(\Delta)$ is trivial locally on $S$.

10.7. In the rest of this section, we use some simplicial topological spaces.

A simplicial topological space is a contravariant functor from the category of non-empty totally ordered finite sets to the category of topological spaces.

A sheaf $F$ on a simplicial topological space $\mathcal{X}$ means a family $\left(F_{n}\right)_{n \geq 0}$, where $F_{n}$ is a sheaf on $\mathcal{X}_{n}=\mathcal{X}(\{0, \ldots, n\})$, endowed with a morphism $a_{F}: \mathcal{X}(a)^{-1}\left(F_{m}\right) \rightarrow F_{n}$ for each $m, n \geq 0$ and for each increasing map $a:\{0, \ldots, m\} \rightarrow\{0, \ldots, n\}$, satisfying $(a b)_{F}=a_{F} \circ \mathcal{X}(a)^{-1}\left(b_{F}\right)$ for any increasing maps $a:\{0, \ldots, m\} \rightarrow\{0, \ldots, n\}$ and $b:\{0, \ldots, l\} \rightarrow\{0, \ldots, m\}$, where $\mathcal{X}(a)^{-1}\left(b_{F}\right)$ is the pull back of $b_{F}$ by $\mathcal{X}(a)$.

For a sheaf $F=\left(F_{n}\right)_{n}$ of abelian groups on a simplicial topological space $\mathcal{X}$, we have a spectral sequence

$$
E_{1}^{i, j}=H^{j}\left(\mathcal{X}_{i}, F_{i}\right) \Rightarrow E_{\infty}^{i}=H^{i}(\mathcal{X}, F) .
$$

For a topological space $V$ and for a topological group $\Gamma$ acting on $V$ continuously, let $[V] /[\Gamma]$ be the following simplicial topological space. We define $([V] /[\Gamma])(\{0, \ldots, n\})=V \times \Gamma^{n}$. For an increasing map $a:\{0, \ldots, m\} \rightarrow$ $\{0, \ldots, n\}$, the corresponding map $V \times \Gamma^{n} \rightarrow V \times \Gamma^{m}$ is given by $\left(v, t_{1}, \ldots, t_{n}\right)$ $\mapsto\left(u, s_{1}, \ldots, s_{m}\right)$, where $u=t_{a(0)} \cdots t_{1} v$ and $s_{i}=t_{a(i)} \cdots t_{a(i-1)+1}$. 
We apply this construction to the cases where $(V, \Gamma)=(V(\Delta)$, $\left.\operatorname{Hom}\left(X, \mathbb{C}^{\times}\right)\right),(V, \Gamma)=\left(V(\Delta)^{\log }, \operatorname{Hom}\left(X, \mathbb{C}^{\times}\right)\right)$and $(V, \Gamma)=\left(V(\Delta)^{\log }\right.$, $\left.\operatorname{Hom}\left(X, \mathbb{S}^{1}\right)\right)$. For any sheaf of abelian groups $F$ on the underlying topological space of $S$, we have

10.7.1. $H^{m}(\bar{V}(\Delta), F) \cong H^{m}\left([V(\Delta)] /\left[\operatorname{Hom}\left(X, \mathbb{C}^{\times}\right)\right], F\right)$.

10.7.2. $H^{m}\left(\left[V(\Delta)^{\log }\right] /\left[\operatorname{Hom}\left(X, \mathbb{C}^{\times}\right)\right], F\right) \cong H^{m}\left(\left[V(\Delta)^{\log }\right] /[\operatorname{Hom}(X\right.$, $\left.\left.\left.\mathbb{S}^{1}\right)\right], F\right)$.

Here we denote the inverse images of $F$ by the same letter $F$.

We prove 10.7.1. In fact, we will prove this not only for a sheaf $F$ of abelian groups on the underlying topological space of $S$, but also for any sheaf $F$ of abelian groups on $(\operatorname{fsan} / S) / \bar{V}(\Delta)$. For $m=0$, since $\bar{V}(\Delta)$ is the quotient of $V(\Delta)$ by the action of $\mathcal{H o m}\left(X, \mathbb{C}^{\times}\right) \times S$ in the category of sheaves on $($ fsan $/ S)$, we have an evident isomorphism 10.7.1. Hence it is sufficient to prove that the functors $H^{m}\left([V(\Delta)] /\left[\operatorname{Hom}\left(X, \mathbb{C}^{\times}\right)\right], \quad\right)$ for $m \geq 1$ applied to sheaves of abelian groups on (fsan $/ S) / \bar{V}(\Delta)$ are "effaceable". Any sheaf of abelian groups $F$ on (fsan $/ S) / \bar{V}(\Delta)$ is embedded in a sheaf $F^{\prime}$ of abelian groups such that the restriction of $F^{\prime}$ on each object of $($ fsan $/ S) / \bar{V}(\Delta)$ is flasque (for example, we can take the sheaf $F^{\prime}(U)=\prod_{u \in U} F_{u}$ ). Furthermore, $F^{\prime}$ is embedded in $F^{\prime \prime}=f_{*} f^{-1} F^{\prime}$, where $f_{*}$ is the direct image functor from the category of sheaves on $($ fsan $/ V(\Delta))=($ fsan $/ S) / V(\Delta)$ to the category of sheaves on $(\operatorname{fsan} / S) / \bar{V}(\Delta)$. By the flasque property of $F^{\prime}$, we have $R^{m} f_{*} f^{-1} F^{\prime}=0$ for $m \geq 1$. Hence in the above spectral sequence (1) for $\mathcal{X}=[V(\Delta)] /\left[\operatorname{Hom}\left(X, \mathbb{C}^{\times}\right)\right]$and for $F^{\prime \prime}$, we have

$$
\begin{aligned}
E_{1}^{i, j} & =H^{j}\left(V(\Delta) \times \Gamma^{i}, F^{\prime \prime}\right) \\
& =H^{j}\left(V(\Delta) \times \Gamma^{i}, R f_{*} f^{-1} F^{\prime}\right) \\
& =H^{j}\left(V(\Delta) \times \Gamma^{i+1}, F^{\prime}\right),
\end{aligned}
$$

where $\Gamma=\operatorname{Hom}\left(X, \mathbb{C}^{\times}\right)$. Hence the group $E_{1}^{i, j}$ is zero if $j \geq 1$ by the flasque property of $F^{\prime}$. As is seen by a standard argument, the complex $E_{1}^{\cdot, 0}$ is acyclic in degree $\geq 1$. Hence $H^{i}\left([V(\Delta)] /\left[\operatorname{Hom}\left(X, \mathbb{C}^{\times}\right)\right], F^{\prime \prime}\right)=0$ for any $i \geq 1$.

Next 10.7.2 follows from the fact that the inclusion $\operatorname{Hom}\left(X, \mathbb{S}^{1}\right) \rightarrow$ $\operatorname{Hom}\left(X, \mathbb{C}^{\times}\right)$is a homotopy equivalence.

For a sheaf $F$ of abelian groups on the underlying topological space of $S$, let

$$
\mathcal{H}^{m}([\bar{V}(\Delta)], F), \mathcal{H}^{m}\left([\bar{V}(\Delta)]^{\log }, F\right), \mathcal{H}^{m}\left([S]^{\log }, F\right)
$$


be the sheaves on $($ fsan $/ S)$ associated to the presheaves $U \mapsto H^{m}\left(\left[V(\Delta) \times_{S}\right.\right.$ $\left.U] /\left[\operatorname{Hom}\left(X, \mathbb{C}^{\times}\right)\right], F\right), U \mapsto H^{m}\left(\left[\left(V(\Delta) \times{ }_{S} U\right)^{\log }\right] /\left[\operatorname{Hom}\left(X, \mathbb{C}^{\times}\right)\right], F\right)$, and $U \mapsto H^{m}\left(U^{\log }, F\right)$, respectively. By 10.7 .1 which is applied on $U$ by taking a compatible chart with $\mathcal{S}$, we have

10.7.3. $\mathcal{H}^{m}(\bar{V}(\Delta), F) \cong \mathcal{H}^{m}([\bar{V}(\Delta)], F)$.

Remark 10.7.4. (This remark is not used in the rest of this paper.) Let $(\text { fsan } / S)^{\log }$ be the site which is denoted as $\left(\mathrm{fs}_{\mathrm{s}} / S\right)^{\log }$ in [6] 3.1.6. For a sheaf $V$ on (fsan $/ S)$, let $\tau^{-1}(V)$ be the inverse image of $V$ on $(\text { fsan } / S)^{\log \text {, }}$ and let $R^{m} \nu_{*}$ be the $m$-th higher direct image functor from the category of sheaves of abelian groups on $(\mathrm{fsan} / S)^{\log } / \tau^{-1}(V)$ to the category of sheaves of abelian groups on $(\mathrm{fsan} / S)$. Let $F$ be a sheaf of abelian groups on the underlying topological space of $S$ (the following in fact holds for any sheaf of

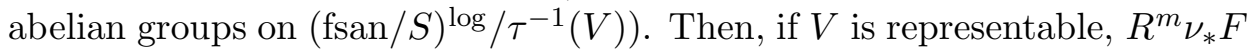
coincides with the sheaf associated to the presheaf $U \mapsto H^{m}\left(\left(U \times_{S} V\right)^{\log }, F\right)$ (hence in the case $V=S$, this coincides with $\mathcal{H}^{m}\left([S]^{\log }, F\right)$ defined above). In the case $V=\bar{V}(\Delta)$, we have

$$
R^{m} \nu_{*} F \cong \mathcal{H}^{m}\left([\bar{V}(\Delta)]^{\log }, F\right) .
$$

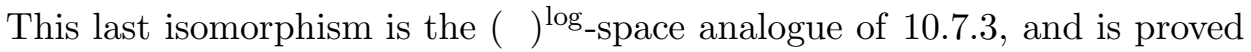
in the same way.

10.8. We say a projective system $\left(H_{\lambda}\right)_{\lambda \in \Lambda}$ in an abelian category with a directed ordered index set $\Lambda$ is essentially zero if for each $\lambda \in \Lambda$, there is $\mu \in \Lambda$ such that $\mu \geq \lambda$ and such that $H_{\mu} \rightarrow H_{\lambda}$ is the zero morphism.

We say a homomorphism $\left(f_{\lambda}: H_{\lambda} \rightarrow H_{\lambda}^{\prime}\right)_{\lambda \in \Lambda}$ of projective systems with directed ordered index set $\Lambda$ is essentially an isomorphism if $\left(\operatorname{Ker}\left(f_{\lambda}\right)\right)_{\lambda}$ and $\left(\operatorname{Coker}\left(f_{\lambda}\right)\right)_{\lambda}$ are essentially zero.

Lemma 10.9. Let $F$ be a sheaf of abelian groups on the underlying topological space of $S$. When $\Delta$ ranges over all finitely generated subcones of $C$, the canonical map $\left(\mathcal{H}^{m}\left([S]^{\log }, F\right) \rightarrow \mathcal{H}^{m}\left([\bar{V}(\Delta)]^{\log }, F\right)\right)_{\Delta}$ is essentially an isomorphism.

Proof. The kernel of the map in 10.9 for $\Delta$ becomes zero as soon as $V(\Delta)$ contains the unit section of $T_{\log }^{(Y)}$.

The essential surjectivity is shown as follows. For each $\Delta$, take a finitely generated subcone $\Delta^{\prime}$ of $C$ containing $\Delta$ satisfying the conditions (i) and 
(ii) in 7.9 (1). The existence of the homotopy ([6] Proposition 3.5.6 (1) (iii)) between the canonical map $V(\Delta)^{\log } \rightarrow V\left(\Delta^{\prime}\right)^{\log }$ and the composition $V(\Delta)^{\log } \rightarrow S^{\log } \times \operatorname{Hom}\left(X, \mathbb{S}^{1}\right) \rightarrow V\left(\Delta^{\prime}\right)^{\log }$ (the second arrow comes from the unit section of $T_{\log }^{(Y)}$ which is contained in $V\left(\Delta^{\prime}\right)$ by the condition (i) in 7.9 (1)) shows the following: The canonical map

$$
H^{m}\left(\left[V\left(\Delta^{\prime}\right)^{\log }\right] /\left[\operatorname{Hom}\left(X, \mathbb{S}^{1}\right)\right], F\right) \longrightarrow H^{m}\left(\left[V(\Delta)^{\log }\right] /\left[\operatorname{Hom}\left(X, \mathbb{S}^{1}\right)\right], F\right)
$$

coincides with the composition

$$
\begin{aligned}
& H^{m}\left(\left[V\left(\Delta^{\prime}\right)^{\log }\right] /\left[\operatorname{Hom}\left(X, \mathbb{S}^{1}\right)\right], F\right) \\
& \quad \longrightarrow H^{m}\left(\left[S^{\log } \times \operatorname{Hom}\left(X, \mathbb{S}^{1}\right)\right] /\left[\operatorname{Hom}\left(X, \mathbb{S}^{1}\right)\right], F\right) \\
& \quad \longrightarrow H^{m}\left(\left[V(\Delta)^{\log }\right] /\left[\operatorname{Hom}\left(X, \mathbb{S}^{1}\right)\right], F\right),
\end{aligned}
$$

where the first arrow comes from the unit section. We have $H^{m}\left(\left[S^{\log } \times\right.\right.$ $\left.\left.\operatorname{Hom}\left(X, \mathbb{S}^{1}\right)\right] /\left[\operatorname{Hom}\left(X, \mathbb{S}^{1}\right)\right], F\right)=H^{m}\left(S^{\log }, F\right)$. This shows that the image of $H^{m}\left(\left[V\left(\Delta^{\prime}\right)^{\log ]} /\left[\operatorname{Hom}\left(X, \mathbb{S}^{1}\right)\right], F\right) \rightarrow H^{m}\left(\left[V(\Delta)^{\log }\right] /\left[\operatorname{Hom}\left(X, \mathbb{S}^{1}\right)\right], F\right)\right.$ is contained in the image of $H^{m}\left(S^{\log }, F\right)$.

Lemma 10.10. Let $F$ be a sheaf of abelian groups on the underlying topological space of $S$. When $\Delta$ ranges over all finitely generated subcones of $C$, the projective system $\left(\mathcal{H}^{1}(\bar{V}(\Delta), F)\right)_{\Delta}$ is essentially zero.

Proof. We first prove

10.10.1. For $m \geq 1$, the map $\left(\mathcal{H}^{m}(\bar{V}(\Delta), F) \rightarrow \mathcal{H}^{m}\left([\bar{V}(\Delta)]^{\log }, F\right)\right)_{\Delta}$ is essentially zero.

In fact, take $\Delta^{\prime}$ as in the proof of 10.9. Then the composition $\mathcal{H}^{m}\left(\bar{V}\left(\Delta^{\prime}\right), F\right) \rightarrow \mathcal{H}^{m}(\bar{V}(\Delta), F) \rightarrow \mathcal{H}^{m}\left([\bar{V}(\Delta)]^{\log }, F\right)$ factors as $\mathcal{H}^{m}\left(\bar{V}\left(\Delta^{\prime}\right)\right.$, $F) \rightarrow \mathcal{H}^{m}(S, F) \rightarrow \mathcal{H}^{m}\left([S]^{\log }, F\right) \rightarrow \mathcal{H}^{m}\left([\bar{V}(\Delta)]^{\log }, F\right)$. Since $\mathcal{H}^{m}(S, F)=$ 0 for $m \geq 1$, this proves that the map $\mathcal{H}^{m}\left(\bar{V}\left(\Delta^{\prime}\right), F\right) \rightarrow \mathcal{H}^{m}\left([\bar{V}(\Delta)]^{\log }, F\right)$ is the zero map.

Now we can prove Lemma 10.10. Let $\tau:\left[V(\Delta)^{\log }\right] /\left[\operatorname{Hom}\left(X, \mathbb{C}^{\times}\right)\right] \rightarrow$ $[V(\Delta)] /\left[\operatorname{Hom}\left(X, \mathbb{C}^{\times}\right)\right]$be the canonical morphism. Then we have $\tau_{*}(F)=$ $F$. Hence the canonical map

$$
\mathcal{H}^{1}(\bar{V}(\Delta), F)=\mathcal{H}^{1}([\bar{V}(\Delta)], F) \rightarrow \mathcal{H}^{1}\left([\bar{V}(\Delta)]^{\log }, F\right)
$$

is injective. By 10.10.1, these injections for varying $\Delta$ form an essentially zero map. This proves Lemma 10.10 . 
Let $G$ be as in 10.2 .

Lemma 10.11. When $\Delta$ ranges over all finitely generated cones of $C$, the projective system of maps $\left(G \rightarrow \mathcal{H}^{0}(\bar{V}(\Delta), G)\right)_{\Delta}$ is essentially an isomorphism.

Proof. The kernel becomes zero as soon as $V(\Delta)$ contains the unit section of $T_{\log }^{(Y)}$.

To prove the essential surjectivity, we apply Lemma 10.10 to $F=$ $\mathcal{H}_{1}(G, \mathbb{Z})$. The exact sequence $0 \rightarrow F \rightarrow \operatorname{Lie}(G) \rightarrow G \rightarrow 0$ induces an exact sequence

$$
\mathcal{H}^{0}(\bar{V}(\Delta), \operatorname{Lie}(G)) \longrightarrow \mathcal{H}^{0}(\bar{V}(\Delta), G) \longrightarrow \mathcal{H}^{1}(\bar{V}(\Delta), F)
$$

for any $\Delta$. Take $\Delta^{\prime} \supset \Delta$ such that $\mathcal{H}^{1}\left(\bar{V}\left(\Delta^{\prime}\right), F\right) \rightarrow \mathcal{H}^{1}(\bar{V}(\Delta), F)$ is the zero map. Then since $\mathcal{H}^{0}(\bar{V}(\Delta), \operatorname{Lie}(G))=\operatorname{Lie}(G)$ if $\Delta$ is sufficiently large by Corollary 10.6, the image of $\mathcal{H}^{0}\left(\bar{V}\left(\Delta^{\prime}\right), G\right) \rightarrow \mathcal{H}^{0}(\bar{V}(\Delta), G)$ is contained in the image of $\operatorname{Lie}(G) \rightarrow \mathcal{H}^{0}(\bar{V}(\Delta), G)$, but the last map factors as $\operatorname{Lie}(G) \rightarrow$ $G \rightarrow \mathcal{H}^{0}(\bar{V}(\Delta), G)$.

10.12. We prove $\mathcal{H o m}\left(T_{\log }^{(Y)} / T, G\right)=0$. We have an injection

$$
\mathcal{H o m}\left(T_{\log }^{(Y)} / T, G\right) \rightarrow \underset{\Delta}{\lim } \mathcal{H}^{0}(\bar{V}(\Delta), G)
$$

By Lemma 10.11, this inverse limit coincides with $G$. Since a homomorphism $T_{\log }^{(Y)} / T \rightarrow G$ sends the unit section of $T_{\log }^{(Y)} / T$ to the unit section of $G$, this injection $\mathcal{H o m}\left(T_{\log }^{(Y)} / T, G\right) \rightarrow G$ is the zero map.

Lemma 10.13. We have an exact sequence

$$
0 \longrightarrow \mathcal{H o m}\left(T_{\log }^{(Y)}, \mathbb{G}_{m}\right) \longrightarrow X \longrightarrow \bar{X} \longrightarrow 0 .
$$

Proof. By 10.12 applied to the case $G=\mathbb{G}_{m}$, the map $\mathcal{H o m}\left(T_{\log }^{(Y)}, \mathbb{G}_{m}\right)$ $\rightarrow \mathcal{H o m}\left(T, \mathbb{G}_{m}\right)=X$ is injective. We prove that the image coincides with $\operatorname{Ker}(X \rightarrow \bar{X})$. For $x \in X$, the map $Y \rightarrow \mathbb{G}_{m, \log } / \mathbb{G}_{m}$ induced by $x$ is the composition $Y \rightarrow T_{\log }^{(Y)} / T \rightarrow \mathbb{G}_{m, \log } / \mathbb{G}_{m}$, where the last arrow is induced by $x$. If $x$ belongs to the image of $\mathcal{H o m}\left(T_{\log }^{(Y)}, \mathbb{G}_{m}\right)$, this last arrow is zero, and hence the image of $x$ in $\bar{X}$ is zero. Let $\varphi$ be a local section of $T_{\log }^{(Y)}$. Then locally there are $y_{1}, y_{2} \in Y$ such that $\left\langle x, y_{1}\right\rangle\left|\left(\varphi(x) \bmod \mathbb{G}_{m}\right)\right|\left\langle x, y_{2}\right\rangle$. If the image of $x$ in $\bar{X}$ vanishes, then $\left\langle x, y_{i}\right\rangle$ vanish for $i=1,2$, and hence $\varphi(x) \bmod \mathbb{G}_{m}$ vanishes. Hence $x$ induces $T_{\log }^{(Y)} \rightarrow \mathbb{G}_{m}$. 
Corollary 10.14. The connecting map $X=\mathcal{H} \operatorname{Hom}\left(T, \mathbb{G}_{m}\right) \rightarrow$ $\mathcal{E} x t\left(T_{\log }^{(Y)} / T, \mathbb{G}_{m}\right)$ factors through the canonical surjection $X \rightarrow \bar{X}$.

LEMMA 10.15. (1) If $\Delta$ is a sufficiently big finitely generated subcone of $C$, the canonical map $\mathbb{G}_{m, \log } / \mathbb{G}_{m} \oplus \bar{X} \rightarrow \mathcal{H}^{0}\left(V(\Delta), \mathbb{G}_{m, \log } / \mathbb{G}_{m}\right)$ is injective.

(2) Let $\Delta$ be a finitely generated subcone of $C$ containing $\mathcal{S}^{\vee} \times\{1\}$ and let $\Delta^{\prime}$ be a finitely generated subcone of $C$ containing $\Delta$ and satisfying the conditions (i) and (ii) of Proposition 7.9 (1). Then the image of $\mathcal{H}^{0}\left(V\left(\Delta^{\prime}\right), \mathbb{G}_{m, \log } / \mathbb{G}_{m}\right) \rightarrow \mathcal{H}^{0}\left(V(\Delta), \mathbb{G}_{m, \log } / \mathbb{G}_{m}\right)$ coincides with the image of the composition $\mathbb{G}_{m, \log } / \mathbb{G}_{m} \oplus \bar{X} \rightarrow \mathcal{H}^{0}\left(V\left(\Delta^{\prime}\right), \mathbb{G}_{m, \log } / \mathbb{G}_{m}\right) \rightarrow$ $\mathcal{H}^{0}\left(V(\Delta), \mathbb{G}_{m, \log } / \mathbb{G}_{m}\right)$.

Proof. Let $f: V(\Delta) \rightarrow V\left(\Delta^{\prime}\right)$ be the canonical map. Then we have

$$
M_{S}^{\mathrm{gp}} / \mathcal{O}_{S}^{\times} \oplus \bar{X} \stackrel{\simeq}{\longrightarrow} f^{-1}\left(M_{V\left(\Delta^{\prime}\right)}^{\mathrm{gp}} / \mathcal{O}_{V\left(\Delta^{\prime}\right)}^{\times}\right) .
$$

The algebraic version of this is Proposition 7.9 (2). This analytic version is proved in the same way. This shows that $H^{0}\left(S, M_{S}^{\mathrm{gp}} / \mathcal{O}_{S}^{\times} \oplus \bar{X}\right) \rightarrow$ $H^{0}\left(V\left(\Delta^{\prime}\right), M_{V\left(\Delta^{\prime}\right)}^{\mathrm{gp}} / \mathcal{O}_{V\left(\Delta^{\prime}\right)}^{\times}\right)$is injective. This proves (1). To prove (2), it is sufficient to prove

$$
H^{0}\left(S, M_{S}^{\mathrm{gp}} / \mathcal{O}_{S}^{\times} \oplus \bar{X}\right) \stackrel{\simeq}{\longrightarrow} H^{0}\left(V(\Delta), M_{S}^{\mathrm{gp}} / \mathcal{O}_{S}^{\times} \oplus \bar{X}\right) .
$$

This follows from the case $P=V(\Delta), Q=S$ of 7.26.1.

10.16. Let $\Delta$ be a finitely generated subcone of $C$. We define a canonical homomorphism

$$
\bar{X} \longrightarrow \mathcal{H}^{2}(\bar{V}(\Delta), \mathbb{Z}(1))
$$

as follows. By 10.14, we have a canonical homomorphism $\bar{X} \rightarrow \mathcal{E} x t\left(T_{\log }^{(Y)} / T\right.$, $\left.\mathbb{G}_{m}\right)$. The canonical map $\mathcal{E} x t\left(T_{\log }^{(Y)} / T, \mathbb{G}_{m}\right) \rightarrow \mathcal{H}^{1}\left(\bar{V}(\Delta), \mathbb{G}_{m}\right)$ and the connecting map $\mathcal{H}^{1}\left(\bar{V}(\Delta), \mathbb{G}_{m}\right) \rightarrow \mathcal{H}^{2}(\bar{V}(\Delta), \mathbb{Z}(1))$ of the exact sequence $0 \rightarrow$ $\mathbb{Z}(1) \rightarrow \mathbb{G}_{a} \stackrel{\exp }{\longrightarrow} \mathbb{G}_{m} \rightarrow 0$ induce the composition $\bar{X} \rightarrow \mathcal{H}^{2}(\bar{V}(\Delta), \mathbb{Z}(1))$.

For any sheaf of abelian groups $F$ on $S$, the homomorphism (1) induces

$$
\bar{X} \otimes F(-1) \longrightarrow \mathcal{H}^{2}(\bar{V}(\Delta), F) .
$$

LEMma 10.17. Let $F$ be a sheaf of abelian groups on the underlying topological space of $S$. Then the projective system of maps $(\bar{X} \otimes F(-1) \rightarrow$ $\left.\mathcal{H}^{2}(\bar{V}(\Delta), F)\right)_{\Delta}$ is essentially an isomorphism. 
Proof. Consider the spectral sequence

$$
E_{2}^{i, j}=\mathcal{H}^{i}\left([\bar{V}(\Delta)], R^{j} \tau_{*} F\right) \Rightarrow E_{\infty}^{i}=\mathcal{H}^{i}\left([\bar{V}(\Delta)]^{\log }, F\right)
$$

and the corresponding spectral sequence for $\Delta^{\prime}$. Here $\mathcal{H}^{i}\left([\bar{V}(\Delta)], R^{j} \tau_{*} F\right)$ is the sheafification of $U \mapsto H^{i}\left(\left[V(\Delta) \times{ }_{S} U\right] /\left[\operatorname{Hom}\left(X, \mathbb{C}^{\times}\right)\right], R^{j} \tau_{*} F\right)$ with $\tau$ the canonical map $\left[\left(V(\Delta) \times{ }_{S} U\right)^{\log ]}\right] /\left[\operatorname{Hom}\left(X, \mathbb{C}^{\times}\right)\right] \rightarrow\left[V(\Delta) \times{ }_{S} U\right] /[\operatorname{Hom}(X$, $\left.\mathbb{C}^{\times}\right)$.

We have

10.17.1. $\mathcal{H}^{1}\left([S]^{\log }, F\right) \cong \mathbb{G}_{m, \log } / \mathbb{G}_{m} \otimes F(-1)$.

This follows from [9] (1.5).

For $\tau: V(\Delta)^{\log } \rightarrow V(\Delta)$, we have $R^{1} \tau_{*} \mathbb{Z}(1)=M_{V(\Delta)}^{\mathrm{gp}} / \mathcal{O}_{V(\Delta)}^{\times}$. Hence the canonical homomorphism $\bar{X} \rightarrow M_{V(\Delta)}^{\mathrm{gp}} / \mathcal{O}_{V(\Delta)}^{\times}$gives a homomorphism

$$
\bar{X} \otimes F(-1) \longrightarrow E_{2}^{0,1}=\mathcal{H}^{0}\left([\bar{V}(\Delta)], R^{1} \tau_{*} F\right) .
$$

We can check that the canonical homomorphism $\bar{X} \otimes F(-1) \rightarrow E_{2}^{2,0}$ in 10.16 coincides with the composition of the above $\bar{X} \otimes F(-1) \rightarrow E_{2}^{0,1}$ with the differential $E_{2}^{0,1} \rightarrow E_{2}^{2,0}$ of the spectral sequence.

By Lemma 10.15, we have

10.17.2. $\left(\left(\mathbb{G}_{m, \log } / \mathbb{G}_{m} \otimes F(-1)\right) \oplus(\bar{X} \otimes F(-1)) \rightarrow \mathcal{H}^{0}\left([\bar{V}(\Delta)], R^{1} \tau_{*} F\right)\right)_{\Delta}$ is essentially an isomorphism.

The spectral sequence gives an exact sequence

$$
\begin{aligned}
\mathcal{H}^{1}\left([\bar{V}(\Delta)]^{\log }, F\right) \longrightarrow \mathcal{H}^{0}([\bar{V}(\Delta)], & \left.R^{1} \tau_{*} F\right) \\
& \longrightarrow \mathcal{H}^{2}([\bar{V}(\Delta)], F) \longrightarrow \mathcal{H}^{2}\left([\bar{V}(\Delta)]^{\log }, F\right) .
\end{aligned}
$$

When $\Delta$ varies, by $10.9,10.10 .1,10.17 .1,10.17 .2$, this gives an exact sequence in the category of projective systems modulo essentially zero systems

$$
\begin{aligned}
\mathbb{G}_{m, \log } / \mathbb{G}_{m} \otimes F(-1) \longrightarrow\left(\left(\mathbb{G}_{m, \log } / \mathbb{G}_{m} \otimes F(-1)\right) \oplus(\bar{X} \otimes F(-1))\right)_{\Delta} \\
\longrightarrow\left(\mathcal{H}^{2}([\bar{V}(\Delta)], F)\right)_{\Delta} \longrightarrow 0 .
\end{aligned}
$$

This proves Lemma 10.17 . 
Lemma 10.18. The composition

$$
\begin{aligned}
& \bar{X} \otimes \mathcal{H}_{1}(G, \mathbb{Z})(-1) \stackrel{10.2(1)}{\cong} \bar{X} \otimes \mathcal{H o m}\left(\mathbb{G}_{m}, G\right) \\
& \stackrel{10.14}{\longrightarrow} \mathcal{E} x t\left(T_{\log }^{(Y)} / T, \mathbb{G}_{m}\right) \otimes \mathcal{H o m}\left(\mathbb{G}_{m}, G\right) \\
& \longrightarrow \mathcal{E} x t\left(T_{\log }^{(Y)} / T, G\right) \longrightarrow \mathcal{H}^{1}(\bar{V}(\Delta), G)
\end{aligned}
$$

gives an essential isomorphism $\left(\bar{X} \otimes \mathcal{H}_{1}(G, \mathbb{Z})(-1) \rightarrow \mathcal{H}^{1}(\bar{V}(\Delta), G)\right)_{\Delta}$.

Proof. Applying $\mathcal{H}^{m}(\bar{V}(\Delta), \quad)$ to the exact sequence $0 \rightarrow \mathcal{H}_{1}(G, \mathbb{Z}) \rightarrow$ $\operatorname{Lie}(G) \rightarrow G \rightarrow 0$, and using $\mathcal{H}^{1}\left(\bar{V}(\Delta), \mathbb{G}_{a}\right)=0$ (Corollary 10.6), we have an injection $\mathcal{H}^{1}(\bar{V}(\Delta), G) \rightarrow \mathcal{H}^{2}\left(\bar{V}(\Delta), \mathcal{H}_{1}(G, \mathbb{Z})\right)$. The composition $\bar{X} \otimes$ $\mathcal{H}_{1}(G, \mathbb{Z})(-1) \rightarrow \mathcal{H}^{1}(\bar{V}(\Delta), G) \rightarrow \mathcal{H}^{2}\left(\bar{V}(\Delta), \mathcal{H}_{1}(G, \mathbb{Z})\right)$ is, when $\Delta$ varies, an essential isomorphism by Lemma 10.17. This proves Lemma 10.18.

10.19. We prove the result on $\mathcal{E} x t\left(T_{\log }^{(Y)} / T, G\right)$ in Theorem 10.3.

We have a canonical homomorphism $\mathcal{E} x t\left(T_{\log }^{(Y)} / T, G\right) \rightarrow \varliminf_{\Delta} \mathcal{H}^{1}(\bar{V}(\Delta)$, $G)$. The latter sheaf is isomorphic to $\bar{X} \otimes \mathcal{H}_{1}(G, \mathbb{Z})(-1)$ by Lemma 10.18 . Since we have a canonical homomorphism $\bar{X} \otimes \mathcal{H}_{1}(G, \mathbb{Z})(-1) \rightarrow \mathcal{E} x t\left(T_{\log }^{(Y)} / T\right.$, $G)$ whose composite with the above canonical homomorphism gives the identity map of $\bar{X} \otimes \mathcal{H}_{1}(G, \mathbb{Z})$, it is sufficient to prove that a section of $\mathcal{E} x t\left(T_{\log }^{(Y)} / T, G\right)$ is trivial if its image in $\varliminf_{\Delta} \mathcal{H}^{1}(\bar{V}(\Delta), G)$ is trivial. For such an extension $0 \rightarrow G \rightarrow F \rightarrow L \rightarrow 0\left(L=T_{\log }^{(Y)} / T\right)$, we have sections $s_{\Delta}: \bar{V}(\Delta) \rightarrow F$ if we neglect the group structure. Replacing $s_{\Delta}$ by $s_{\Delta} s_{\Delta}(e)^{-1}$, we may assume that $s_{\Delta}$ sends the unit section $e$ to 1 whenever $\bar{V}(\Delta)$ contains $e$. Let $\hat{s}_{\Delta}: \bar{V}(\Delta) \rightarrow F$ be the restriction of $s_{\Delta^{\prime}}$ for a sufficiently large $\Delta^{\prime}$, which is independent of the choice of $\Delta^{\prime}$ by Lemma 10.11. Then the $\hat{s}_{\Delta}$ s glue together into a section $s: L \rightarrow F$ sending $e$ to 1 . Again by Lemma 10.11 (which we apply by using $\Delta \times \Delta$ in the place of $\Delta$ ), the restricted morphism $s(x y) s(x)^{-1} s(y)^{-1}: \bar{V}(\Delta) \times \bar{V}(\Delta) \rightarrow G$ comes from $a \in G$. Since $s(e)=1$, we have $a=1$, so that $s(x y)=s(x) s(y)$ on $\bar{V}(\Delta)$ for any $\Delta$. Thus $s$ preserves the group structure.

10.20. We prove the statements on $\mathcal{H o m}\left(T_{\log }^{(Y)}, G\right)$ and $\mathcal{E} x t\left(T_{\log }^{(Y)}, G\right)$ in Theorem 10.3.

By the exact sequence $0 \rightarrow T \rightarrow T_{\log }^{(Y)} \rightarrow T_{\log }^{(Y)} / T \rightarrow 0$, we have an exact 
sequence

$$
\begin{aligned}
& 0 \longrightarrow \mathcal{H o m}\left(T_{\log }^{(Y)} / T, G\right) \longrightarrow \mathcal{H o m}\left(T_{\log }^{(Y)}, G\right) \longrightarrow \mathcal{H o m}(T, G) \\
& \longrightarrow \mathcal{E} x t\left(T_{\log }^{(Y)} / T, G\right) \longrightarrow \mathcal{E} x t\left(T_{\log }^{(Y)}, G\right) \longrightarrow \mathcal{E} x t(T, G)
\end{aligned}
$$

By 10.12 and 10.19, this is rewritten as

$$
\begin{aligned}
0 \longrightarrow 0 \longrightarrow \mathcal{H o m}\left(T_{\log }^{(Y)}, G\right) \longrightarrow X \otimes \mathcal{H}_{1}(G, \mathbb{Z})(-1) \\
\longrightarrow \bar{X} \otimes \mathcal{H}_{1}(G, \mathbb{Z})(-1) \longrightarrow \mathcal{E x t}\left(T_{\log }^{(Y)}, G\right) \longrightarrow \mathcal{E x t}(T, G)
\end{aligned}
$$

Hence we obtain the statement for $\mathcal{H o m}\left(T_{\log }^{(Y)}, G\right)$. To prove $\mathcal{E} x t\left(T_{\log }^{(Y)}, G\right)=$ 0 , it is sufficient to show $\mathcal{E} x t(T, G)=0$ and hence it is sufficient to prove $\mathcal{E} x t\left(\mathbb{G}_{m}, G\right)=0$. By the exact sequence $0 \rightarrow \mathbb{Z}(1) \rightarrow \mathbb{G}_{a} \rightarrow \mathbb{G}_{m} \rightarrow 0$, we have an exact sequence

$$
\mathcal{H o m}\left(\mathbb{G}_{a}, G\right) \longrightarrow \mathcal{H o m}(\mathbb{Z}(1), G) \longrightarrow \mathcal{E} x t\left(\mathbb{G}_{m}, G\right) \longrightarrow \mathcal{E} x t\left(\mathbb{G}_{a}, G\right) .
$$

The map $\mathcal{H o m}\left(\mathbb{G}_{a}, G\right) \rightarrow \mathcal{H o m}(\mathbb{Z}(1), G)$ is surjective because for a local section $a$ of $G, a=\exp (b)$ locally for some local section $b$ of Lie $(G)$, and the homomorphism $\mathbb{G}_{a} \rightarrow G ; t \mapsto \exp \left((2 \pi i)^{-1}(t b)\right)$ induces $\mathbb{Z}(1) \rightarrow G$ which sends $2 \pi i$ to $a$. It remains to prove $\mathcal{E} x t\left(\mathbb{G}_{a}, G\right)=0$. Consider an exact sequence $0 \rightarrow G \rightarrow F \rightarrow \mathbb{G}_{a} \rightarrow 0$. By taking Lie, we have a commutative diagram of exact sequences

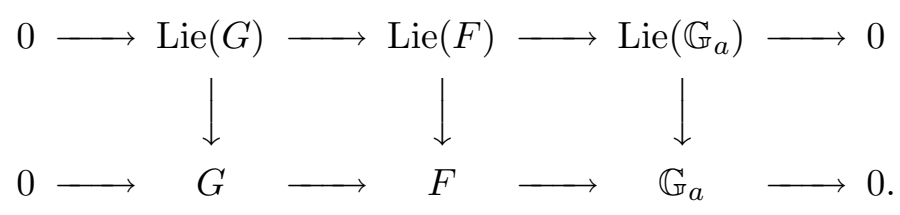

Here the vertical arrows are the exponential maps. Since the exponential map of $\mathbb{G}_{a}$ is an isomorphism, this shows that the extension $0 \rightarrow G \rightarrow F \rightarrow$ $\mathbb{G}_{a} \rightarrow 0$ comes from an exact sequence $0 \rightarrow \operatorname{Lie}(G) \rightarrow \operatorname{Lie}(F) \rightarrow \operatorname{Lie}\left(\mathbb{G}_{a}\right) \rightarrow$ 0 of locally free modules on $S$ by pushing forward via $\exp : \operatorname{Lie}(G) \rightarrow G$, and hence splits locally on $S$.

\section{$\S 11$. Proofs of Section 4, II}

In this section, $S$ denotes an fs $\log$ scheme over $\mathbb{C}$ locally of finite type. We will prove 4.10 . 
Proposition 11.1. Let $M=\left[Y \rightarrow G_{\log }\right]$ be a log 1-motif over $S$ of type $(X, Y)$ such that the associated pairing $X \times Y \rightarrow\left(\mathbb{G}_{m, \log } / \mathbb{G}_{m}\right)_{S}$ is admissible and non-degenerate. Let $A=G_{\log }^{(Y)} / Y$.

(1) $A^{\text {an }}$ is a log complex torus over $S^{\text {an }}$ in the sense of [6] (cf. 4.11.4).

(2) If $M$ is polarizable étale locally on $S, A^{\text {an }}$ is polarizable locally on $S^{\text {an }}$ in the sense of [6] (cf. 4.11.5).

The log complex tori obtained in this way are of very special type. They have to be "with constant degeneration".

11.2. We prove Proposition 11.1 (1). It is sufficient to prove that locally on $S^{\text {an }}$, there are finitely generated free $\mathbb{Z}$-modules $X^{\prime}, Y^{\prime}$, surjective homomorphisms $X^{\prime} \rightarrow X, Y^{\prime} \rightarrow Y$, and a $\mathbb{Z}$-bilinear form $\langle\rangle:, X^{\prime} \times$ $Y^{\prime} \rightarrow\left(\mathbb{G}_{m, \log }\right)_{S}$ satisfying the following conditions (1) and (2), such that $A^{\text {an }} \cong \mathcal{H o m}\left(X^{\prime}, \mathbb{G}_{m, \log }\right)^{\left(Y^{\prime}\right)} / Y^{\prime}$.

(1) The diagram

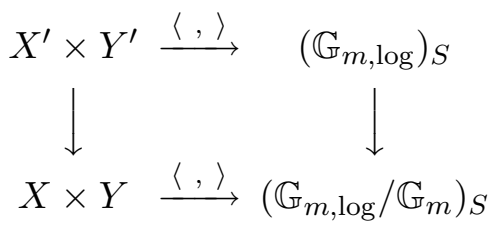

is commutative.

(2) Let $X^{\prime \prime}=\operatorname{Ker}\left(X^{\prime} \rightarrow X\right)$ and $Y^{\prime \prime}=\operatorname{Ker}\left(Y^{\prime} \rightarrow Y\right)$. Then the induced pairing $\langle\rangle:, X^{\prime \prime} \times Y^{\prime \prime} \rightarrow\left(\mathbb{G}_{m}\right)_{S}$ has the following property. For each $s \in S^{\text {an }}$, the pairing of $\mathbb{R}$-vector spaces

$$
\left(\mathbb{R} \otimes X^{\prime \prime}\right) \times\left(\mathbb{R} \otimes Y^{\prime \prime}\right) \longrightarrow \mathbb{R} ;(x, y) \longmapsto \log (|\langle x, y\rangle(s)|) \quad\left(x \in X^{\prime \prime}, y \in Y^{\prime \prime}\right)
$$

is non-degenerate.

We first construct $X^{\prime \prime}, Y^{\prime \prime}$ and a pairing $X^{\prime \prime} \times Y^{\prime \prime} \rightarrow\left(\mathbb{G}_{m}\right)_{S}$.

Since $\mathcal{O}_{S^{\text {an }}} \otimes_{\mathbb{Z}} \mathcal{H}_{1}\left(B^{\text {an }}, \mathbb{Z}\right) \rightarrow \operatorname{Lie}\left(B^{\text {an }}\right)$ is surjective, we can find locally on $S^{\text {an }}$ a locally constant subgroup sheaf of $\mathcal{H}_{1}\left(B^{\text {an }}, \mathbb{Z}\right)$ which we denote by $\left(X^{\prime \prime}\right)^{*}$ satisfying the following conditions (3) and (4).

(3) The homomorphism $\mathcal{O}_{S^{\text {an }}} \otimes_{\mathbb{Z}}\left(X^{\prime \prime}\right)^{*} \rightarrow \operatorname{Lie}\left(B^{\text {an }}\right)$ is an isomorphism.

(4) $\mathcal{H}_{1}\left(B^{\text {an }}, \mathbb{Z}\right) /\left(X^{\prime \prime}\right)^{*}$ is torsion free.

Define

$$
X^{\prime \prime}=\mathcal{H o m}\left(\left(X^{\prime \prime}\right)^{*}, \mathbb{Z}\right)(1), \quad Y^{\prime \prime}=\mathcal{H}_{1}\left(B^{\text {an }}, \mathbb{Z}\right) /\left(X^{\prime \prime}\right)^{*} .
$$


We have an exact sequence

$$
0 \longrightarrow \mathcal{H o m}\left(X^{\prime \prime}, \mathbb{Z}\right)(1) \longrightarrow \mathcal{H}_{1}\left(B^{\text {an }}, \mathbb{Z}\right) \longrightarrow Y^{\prime \prime} \longrightarrow 0 .
$$

Since we have a perfect pairing of finitely generated free $\mathbb{Z}$-modules

$$
\mathcal{H}_{1}\left(B^{\text {an }}, \mathbb{Z}\right) \times \mathcal{H}_{1}\left(\left(B^{*}\right)^{\text {an }}, \mathbb{Z}\right) \longrightarrow \mathbb{Z}(1),
$$

(5) gives an exact sequence

$$
0 \longrightarrow \mathcal{H o m}\left(Y^{\prime \prime}, \mathbb{Z}(1)\right) \longrightarrow \mathcal{H}_{1}\left(\left(B^{*}\right)^{\text {an }}, \mathbb{Z}\right) \longrightarrow X^{\prime \prime} \longrightarrow 0 .
$$

The homomorphism $\mathcal{O}_{S^{\text {an }}} \otimes_{\mathbb{Z}}\left(Y^{\prime \prime}\right)^{*} \rightarrow \operatorname{Lie}\left(\left(B^{*}\right)^{\text {an }}\right)$ is an isomorphism, where $\left(Y^{\prime \prime}\right)^{*}=\mathcal{H o m}\left(Y^{\prime \prime}, \mathbb{Z}\right)(1)$.

The isomorphism $\mathcal{H o m}\left(X^{\prime \prime}, \mathbb{G}_{a}\right) \stackrel{\simeq}{\rightarrow} \operatorname{Lie}\left(B^{\text {an }}\right)$ induces a homomorphism of subgroups $\mathcal{H o m}\left(X^{\prime \prime}, \mathbb{Z}\right)(1)=\left(X^{\prime \prime}\right)^{*} \rightarrow \mathcal{H}_{1}\left(B^{\text {an }}, \mathbb{Z}\right)$ which is injective with cokernel $Y^{\prime \prime}$, and hence it induces a homomorphism of quotient groups $\mathcal{H o m}\left(X^{\prime \prime}, \mathbb{G}_{m}\right) \rightarrow B^{\text {an }}$ which is surjective with kernel $Y^{\prime \prime}$. Thus we have an exact sequence (a multiplicative presentation of $B^{\text {an }}$ )

$$
0 \longrightarrow Y^{\prime \prime} \longrightarrow \mathcal{H o m}\left(X^{\prime \prime}, \mathbb{G}_{m}\right) \longrightarrow B^{\text {an }} \longrightarrow 0 .
$$

The same construction using (6) gives an exact sequence

$$
0 \longrightarrow X^{\prime \prime} \longrightarrow \mathcal{H o m}\left(Y^{\prime \prime}, \mathbb{G}_{m}\right) \longrightarrow\left(B^{*}\right)^{\text {an }} \longrightarrow 0
$$

It can be shown that the pairing $X^{\prime \prime} \times Y^{\prime \prime} \rightarrow\left(\mathbb{G}_{m}\right)_{S}$ obtained in $(7)$ coincides with the one obtained in (8), and that this pairing satisfies the condition (2).

We define $X^{\prime}$ and $Y^{\prime}$ by the following cartesian diagrams $(*)$ with which we have commutative diagrams of exact sequences

(9)
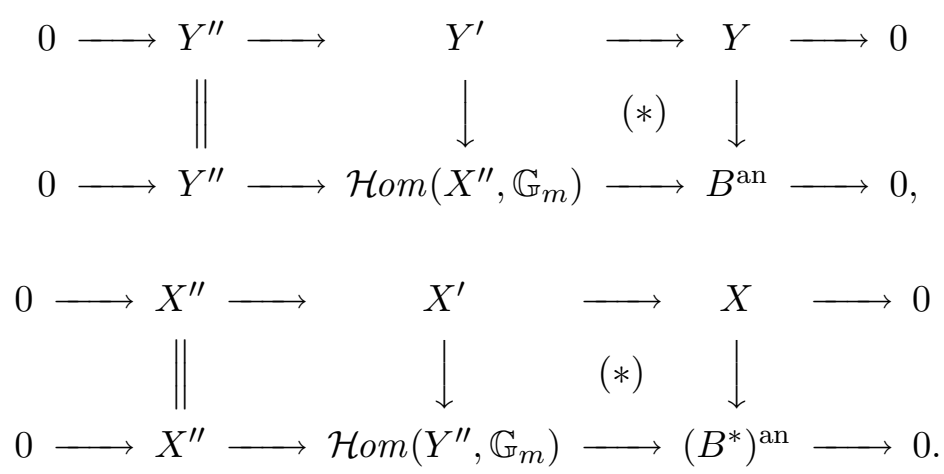
We have exact sequences

$$
\begin{gathered}
0 \longrightarrow Y^{\prime \prime} \longrightarrow \mathcal{H o m}\left(X^{\prime}, \mathbb{G}_{m}\right) \longrightarrow G^{\text {an }} \longrightarrow 0 \\
0 \longrightarrow X^{\prime \prime} \longrightarrow \mathcal{H o m}\left(Y^{\prime}, \mathbb{G}_{m}\right) \longrightarrow\left(G^{*}\right)^{\text {an }} \longrightarrow 0
\end{gathered}
$$

Here $\mathcal{H o m}\left(Y^{\prime}, \mathbb{G}_{m}\right) \rightarrow\left(G^{*}\right)^{\text {an }}$ is defined as the composition

$$
\begin{aligned}
\mathcal{H o m}\left(Y^{\prime}, \mathbb{G}_{m}\right) \longrightarrow \mathcal{E} x t\left(\left[Y^{\prime} \rightarrow \mathcal{H o m}\left(X^{\prime \prime}, \mathbb{G}_{m}\right)\right], \mathbb{G}_{m}\right) \\
\\
\quad \cong \mathcal{E} x t\left(\left[Y \rightarrow B^{\mathrm{an}}\right], \mathbb{G}_{m}\right)=\left(G^{*}\right)^{\text {an }},
\end{aligned}
$$

and $\mathcal{H o m}\left(X^{\prime}, \mathbb{G}_{m}\right) \rightarrow G^{\text {an }}$ is defined similarly.

Let $\left(X^{\prime}\right)^{*}=\mathcal{H o m}\left(X^{\prime}, \mathbb{Z}\right)(1), X^{*}=\mathcal{H o m}(X, \mathbb{Z})(1)$. The exact sequence (11) induces an injective homomorphism $\left(X^{\prime}\right)^{*}=\mathcal{H}_{1}\left(\mathcal{H o m}\left(X^{\prime}, \mathbb{G}_{m}\right), \mathbb{Z}\right) \rightarrow$ $\mathcal{H}_{1}\left(G^{\text {an }}, \mathbb{Z}\right)$, and we have a commutative diagram of exact sequences and its dual version

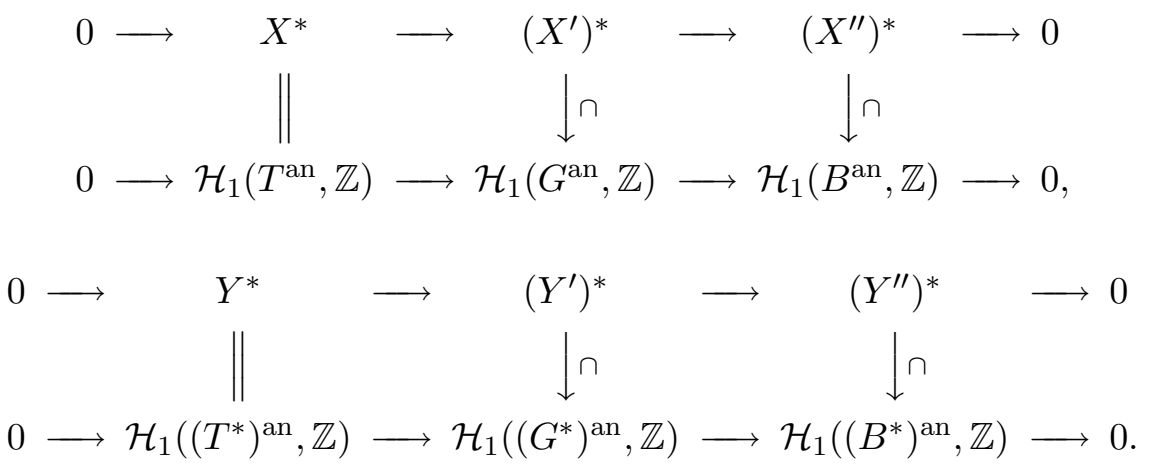

Now we define the pairing $\langle\rangle:, X^{\prime} \times Y^{\prime} \rightarrow\left(\mathbb{G}_{m, \log }\right)_{S}$ as follows.

Let $\mathcal{H o m}\left(X^{\prime}, \mathbb{G}_{m, \log }\right)_{0}$ be the subgroup sheaf of $\mathcal{H o m}\left(X^{\prime}, \mathbb{G}_{m, \log }\right)$ consisting of all local sections which send $X^{\prime \prime}$ into $\mathbb{G}_{m}$. Then the homomor$\operatorname{phism} \mathcal{H o m}\left(X^{\prime}, \mathbb{G}_{m}\right) \rightarrow G^{\text {an }}$ induces a homomorphism $\mathcal{H o m}\left(X^{\prime}, \mathbb{G}_{m, \log }\right)_{0} \rightarrow$ $\left(G_{\log }\right)^{\text {an }}$. This and the similar construction by using $\mathcal{H o m}\left(Y^{\prime}, \mathbb{G}_{m}\right) \rightarrow$ $\left(G^{*}\right)^{\text {an }}$ give cartesian diagrams

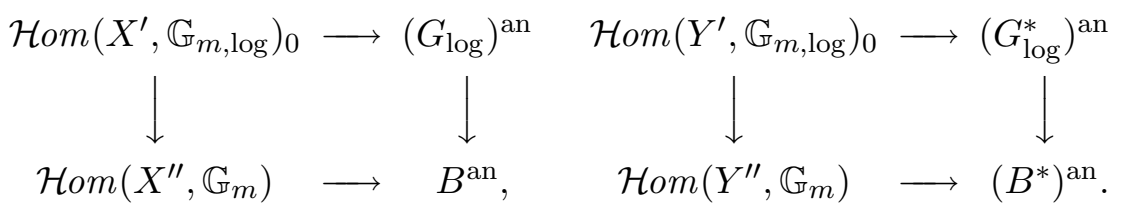

By the left cartesian diagram, the pair of the homomorphisms $Y^{\prime} \rightarrow Y \rightarrow$ $\left(G_{\log }\right)^{\text {an }}$ and $Y^{\prime} \rightarrow \mathcal{H o m}\left(X^{\prime \prime}, \mathbb{G}_{m}\right)$ induces a homomorphism $Y^{\prime} \rightarrow \mathcal{H o m}\left(X^{\prime}\right.$, 
$\left.\mathbb{G}_{m, \log }\right)_{0}$. Similarly, by the right cartesian diagram, the pair of the homomorphisms $X^{\prime} \rightarrow X \rightarrow\left(G_{\log }^{*}\right)$ an (here the last arrow comes from the dual $\log 1$-motif $M^{*}=\left[X \rightarrow G_{\mathrm{log}}^{*}\right]$ of $\left.M\right)$ and $X^{\prime} \rightarrow \mathcal{H o m}\left(Y^{\prime \prime}, \mathbb{G}_{m}\right)$ induces a homomorphism $X^{\prime} \rightarrow \mathcal{H o m}\left(Y^{\prime}, \mathbb{G}_{m, \log }\right)_{0}$. It can be shown that the pairings $X^{\prime} \times Y^{\prime} \rightarrow\left(\mathbb{G}_{m, \log }\right)_{S}$ obtained in these two ways are the same. The diagram

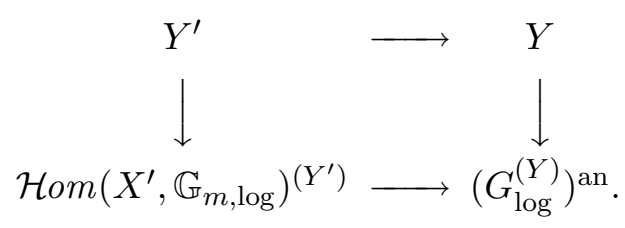

is cartesian. Hence we have

$$
A^{\text {an }}=\left(G_{\log }^{(Y)}\right)^{\text {an }} / Y \cong \mathcal{H} \text { om }\left(X^{\prime}, \mathbb{G}_{m, \log }\right)^{\left(Y^{\prime}\right)} / Y^{\prime} .
$$

This proves that $A^{\text {an }}$ is a $\log$ complex torus over $S^{\text {an }}$.

11.3. We prove Proposition 11.1 (2). Assume that we have a polarization $h: M=\left[Y \rightarrow G_{\log }\right] \rightarrow M^{*}=\left[X \rightarrow G_{\log }^{*}\right]$. This gives a polarization $h: B \rightarrow B^{*}$. The composition $\mathcal{H}_{1}\left(B^{\text {an }}, \mathbb{Z}\right) \times \mathcal{H}_{1}\left(B^{\text {an }}, \mathbb{Z}\right) \rightarrow$ $\mathcal{H}_{1}\left(B^{\text {an }}, \mathbb{Z}\right) \times \mathcal{H}_{1}\left(\left(B^{*}\right)^{\text {an }}, \mathbb{Z}\right) \rightarrow \mathbb{Z}(1)$ induced by $h$ is an anti-symmetric pairing. We can find $\left(X^{\prime \prime}\right)^{*}$ in 11.2 such that this pairing kills $\left(X^{\prime \prime}\right)^{*} \times\left(X^{\prime \prime}\right)^{*}$. Then the pairing induces $\left(X^{\prime \prime}\right)^{*} \times Y^{\prime \prime}=\left(X^{\prime \prime}\right)^{*} \times \mathcal{H}_{1}\left(B^{\text {an }}, \mathbb{Z}\right) /\left(X^{\prime \prime}\right)^{*} \rightarrow \mathbb{Z}(1)$, that is, a homomorphism $\phi: Y^{\prime \prime} \rightarrow X^{\prime \prime}$. The following diagram is commutative:

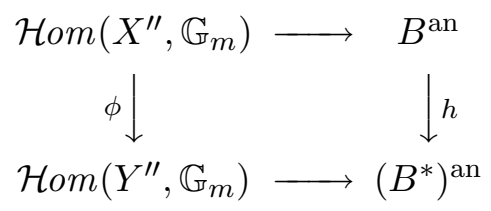

Since $h: B \rightarrow B^{*}$ is a polarization, we have:

(1) $\langle\phi(y), z\rangle=\langle\phi(z), y\rangle$ for any $y, z \in Y^{\prime \prime}$.

(2) For each $s \in S^{\text {an }}$, the induced symmetric bilinear form

$$
\left(\mathbb{R} \otimes Y^{\prime \prime}\right) \times\left(\mathbb{R} \otimes Y^{\prime \prime}\right) \longrightarrow \mathbb{R} ;(y, z) \longmapsto-\log (|\langle\phi(y), z\rangle(s)|) \quad\left(y, z \in Y^{\prime \prime}\right)
$$

is positive definite.

Now a homomorphism $\phi: Y^{\prime} \rightarrow X^{\prime}$ is defined (by the definitions of $X^{\prime}$ and $Y^{\prime}$ as fiber products in (9) and (10) in 11.2) by $h: Y \rightarrow X, h: B \rightarrow B^{*}$, 
and $\mathcal{H o m}\left(X^{\prime \prime}, \mathbb{G}_{m}\right) \rightarrow \mathcal{H o m}\left(Y^{\prime \prime}, \mathbb{G}_{m}\right)\left(\right.$ by $\left.\phi: Y^{\prime \prime} \rightarrow X^{\prime \prime}\right)$. The diagram

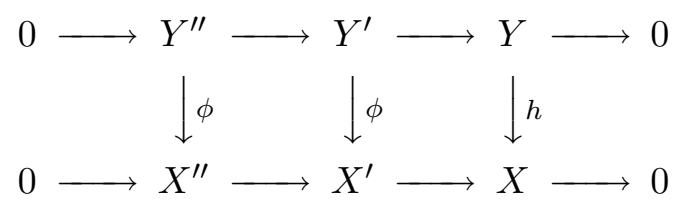

is commutative.

To prove that $\phi: Y^{\prime} \rightarrow X^{\prime}$ gives a polarization of $A^{\text {an }}$ in the sense of [6] (cf. 4.11), it is sufficient to prove that the following conditions (i)-(iii) are satisfied (cf. 4.11.3, [6] 1.2.7).

(i) $\phi: Y^{\prime} \rightarrow X^{\prime}$ is injective and the cokernel of $\phi$ is finite.

(ii) $\langle\phi(y), z\rangle=\langle\phi(z), y\rangle$ for any $y, z \in Y^{\prime}$.

(iii) For any $y \in Y^{\prime},\langle\phi(y), y\rangle \in M_{S}$ in $M_{S}^{g p}$. For any $y \in Y^{\prime} \backslash\{0\}$, the map $\alpha: M_{S} \rightarrow \mathcal{O}_{S}$ sends $\langle\phi(y), y\rangle$ to a function on $S$ whose values are always of absolute value $<1$.

The condition (i) is satisfied as is seen from the above commutative diagram and from the injectivity and the finiteness of the cokernel of the homomorphisms $\phi: Y^{\prime \prime} \rightarrow X^{\prime \prime}$ and $h: Y \rightarrow X$. The condition (iii) is satisfied as is seen from the above property (2) of $\phi: Y^{\prime \prime} \rightarrow X^{\prime \prime}$ and from the property (c) of $h: Y \rightarrow X$ in Definition 2.8. It remains to prove that the condition (ii) is satisfied. That is, it is sufficient to prove that the composition $Y^{\prime} \rightarrow \mathcal{H o m}\left(X^{\prime}, \mathbb{G}_{m, \log }\right)_{0} \stackrel{\phi}{\rightarrow} \mathcal{H o m}\left(Y^{\prime}, \mathbb{G}_{m, \log }\right)_{0}$ coincides with the composition $Y^{\prime} \stackrel{\phi}{\rightarrow} X^{\prime} \rightarrow \mathcal{H o m}\left(Y^{\prime}, \mathbb{G}_{m, \log }\right)_{0}$. Since $\mathcal{H o m}\left(Y^{\prime}, \mathbb{G}_{m, \log }\right)_{0} \subset$ $\left(G_{\log }^{*}\right)^{\text {an }} \times \mathcal{H} \operatorname{Hom}\left(Y^{\prime \prime}, \mathbb{G}_{m}\right)$, this is reduced to the commutativity of the two diagrams

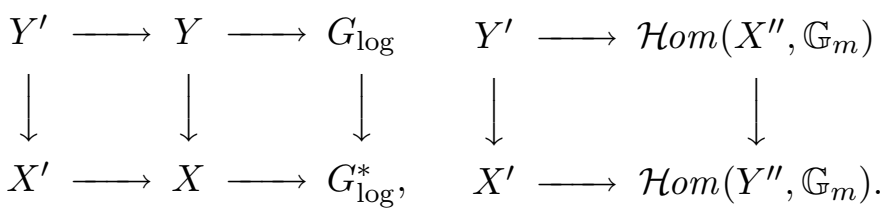

(the commutativity of the right diagram is evident by the definition of $Y^{\prime} \rightarrow$ $\left.X^{\prime}\right)$.

This completes the proof of Proposition 11.1.

11.4. In the proof of Theorem 4.10, we will use the following lemmas 11.4.1 and 11.4.3. 
LEMMA 11.4.1. Let $S$ be an fs log scheme whose underlying scheme is of finite type over a field $k$ of characteristic 0 . Then there is a finite sequence of $f$ s log schemes $S_{n} \stackrel{f_{n}}{\longrightarrow} S_{n-1} \stackrel{f_{n-1}}{\longrightarrow} \cdots \stackrel{f_{1}}{\longrightarrow} S_{0}=S$ satisfying the following conditions (i) and (ii).

(i) Each $f_{i}(1 \leq i \leq n)$ is either proper surjective or strict étale surjective. (Here " $f_{i}$ is proper" means that the underlying morphism of schemes of $f_{i}$ is proper, and " $f_{i}$ is strict" means that the log structure of $S_{i}$ coincides with the pull back of the log structure of $S_{i-1}$ via $f_{i}$ ).

(ii) Let $P$ be any connected component of $S_{n}$. Then the underlying scheme of $P$ is smooth over $k$, and there is a divisor $D$ on the underlying scheme of $P$ with normal crossings such that the log structure of $P$ coincides with

$$
M_{P, D, r}=\mathbb{N}^{r} \times\left\{f \in \mathcal{O}_{P} \mid f \text { is invertible outside } D\right\} \subset \mathbb{N}^{r} \times \mathcal{O}_{P}
$$

for some $r \geq 0$ with $\alpha: M_{P, D, r} \rightarrow \mathcal{O}_{P}$ which sends $(m, f)$ to $f$ if $m=0$ and to 0 otherwise.

Proof. We may assume that there are an fs monoid $N$ such that $N^{\times}=$ $\{1\}$ and a chart $N \rightarrow M_{S}$. Take a regular subdivision of $\operatorname{Hom}(N, \mathbb{N})$ and the proper log étale scheme $S^{\prime}$ over $S$ corresponding to it ([8] Proposition (9.9); in fact, its statement should be modified slightly, which can be easily done by considering the fs or integrality condition). Replacing $S$ by $S^{\prime}$ and working locally, we may assume that the $\log$ structure of $S$ is associated to a homomorphism $h: \mathbb{N}^{n} \rightarrow \mathcal{O}_{S}$ for some $n \geq 0$. By replacing $S$ by the disjoint union of irreducible components of $S$ endowed with the reduced scheme structures, we may assume that the underlying scheme of $S$ is integral. Then changing the order of the canonical base of $\mathbb{N}^{n}$ if necessary, we have $r$ such that $0 \leq r \leq n$ and such that for $m \in \mathbb{N}^{n}, h(m) \neq 0$ if and only if $m_{i}=0$ for all $1 \leq i \leq r$. Let $m$ be the element of $\mathbb{N}^{n}$ such that $m_{i}=0$ for $1 \leq i \leq r$ and such that $m_{i}=1$ for $r<i \leq n$. By the desingularization of Hironaka, there is a proper birational morphism of schemes $S^{\prime} \rightarrow S$ such that $S^{\prime}$ is smooth over $k$ and such that the closed subscheme of $S^{\prime}$ defined by $h(m)$ is a normal crossing divisor $D$ on $S^{\prime}$. We can replace $S$ by $S^{\prime}$ endowed with the $\log$ structure $M_{S^{\prime}, D, r}$.

Remark 11.4.2. In Lemma 11.4.1, we can always take $r=1$. Indeed, for any $r \geq 0$, we have a morphism of fs log schemes $\left(P, M_{P, D, 1}\right) \rightarrow$ $\left(P, M_{P, D, r}\right)$ associated to the homomorphism $\mathbb{N}^{r} \rightarrow \mathbb{N} ;\left(m_{i}\right) \mapsto \sum_{i} m_{i}$. 
Lemma 11.4.3. Let $f: P \rightarrow Q$ be a continuous map of topological spaces, let $R$ be a ring, and let $F$ be a sheaf of $R$-modules on $Q$ all of whose stalks are of finite presentation as $R$-modules. Assume that $f^{-1}(F)$ is locally constant and assume that $f$ is a surjective closed map. Then $F$ is locally constant.

Proof. Let $s \in Q$. Since $F_{s}$ is of finite presentation, there is a homomorphism $h:\left.F_{s} \rightarrow F\right|_{U}$ for some open neighborhood $U$ of $s$ such that the composition $F_{s} \rightarrow F(U) \rightarrow F_{s}$ is the identity map. We prove that $h$ induces an isomorphism $\left.F_{s} \cong F\right|_{U^{\prime}}$ for some open neighborhood $U^{\prime} \subset U$ of $s$. Since $f^{-1}(F)$ is locally constant, there is an open neighborhood $V$ of $f^{-1}(s)$ in $f^{-1}(U)$ such that $h$ induces an isomorphism $\left.F_{s} \cong f^{-1}(F)\right|_{V}$. Let $C$ be the complement of $V$ in $P$, and let $C^{\prime}$ be the image of $C$ in $Q$. Since $C$ is closed and $f$ is a closed map, $C^{\prime}$ is closed. Let $U^{\prime}=Q \backslash C^{\prime}$. Then, since $U^{\prime} \subset f(V)$ by the surjectivity of $f$, the map $\left.F_{s} \rightarrow F\right|_{U^{\prime}}$ is an isomorphism.

11.5. Proof of Theorem 4.10. We prove that if the underlying scheme of $S$ is locally of finite type over $\mathbb{C}$ and $A$ is a log abelian variety over $S$, then $A^{\text {an }}$ is a $\log$ abelian variety over $S^{\text {an }}$ in the sense of [6] (cf. 4.11.6).

By Proposition 11.1 (2), it is sufficient to prove that $A^{\text {an }}$ is a log complex torus.

We may assume that we have an admissible pairing $X \times Y \rightarrow M_{S}^{\mathrm{gp}} / \mathcal{O}_{S}^{\times}$ in 4.1.2 globally on $S$.

Let $G$ be the semi-abelian part of $A(4.4)$, and consider the exact sequence

$$
0 \longrightarrow G^{\text {an }} \longrightarrow A^{\text {an }} \longrightarrow L / \bar{Y} \longrightarrow 0, \quad \text { where } L=\mathcal{H o m}\left(X, \mathbb{G}_{m, \log } / \mathbb{G}_{m}\right)^{(Y)},
$$

and let

$$
0 \longrightarrow G^{\text {an }} \longrightarrow \widetilde{A}^{\text {an }} \longrightarrow L \longrightarrow 0
$$

be the pull back of it by $L \rightarrow L / \bar{Y}$. Let $\xi \in \bar{X} \otimes \mathcal{H}_{1}\left(G^{\text {an }}, \mathbb{Z}\right)(-1)$ be the element corresponding to the extension (1) via the isomorphism $\mathcal{E} x t\left(L, G^{\text {an }}\right) \cong$ $\bar{X} \otimes \mathcal{H}_{1}\left(G^{\text {an }}, \mathbb{Z}\right)(-1)$ in Theorem 10.3. At each $s \in S^{\text {an }}$, if $T / s$ denotes the torus part of $G \times{ }_{S} s$, the stalk $\xi_{s}$ of $\xi$ at $s$ belongs to $\bar{X}_{s} \otimes H_{1}\left((T / s)^{\text {an }}, \mathbb{Z}\right)(-1)$ and induces an isomorphism $H_{1}\left((T / s)^{\text {an }}, \mathbb{Z}\right) \cong \operatorname{Hom}\left(\bar{X}_{s}, \mathbb{Z}\right)(1)$.

Next we prove 
11.5.1. Let $W$ be an fs $\log$ analytic space over $S^{\text {an }}$ such that $M_{W} / \mathcal{O}_{W}^{\times}$ is locally constant. Then the pull back of $\mathcal{H}_{1}\left(G^{\text {an }}, \mathbb{Z}\right)$ to $($ fsan $/ W)$ is locally constant.

Proof. As is easily seen, locally on $W$, there are an fs log scheme $S^{\prime}$ over $S$ such that $M_{S^{\prime}} / \mathcal{O}_{S^{\prime}}^{\times}$is constant and a morphism $W \rightarrow\left(S^{\prime}\right)^{\text {an }}$ of fs $\log$ analytic spaces over $S^{\text {an }}$. By Theorem $4.6(2)$, the pull back of $A$ to $S^{\prime}$ is a $\log$ abelian variety with constant degeneration. Hence $G \times{ }_{S} S^{\prime}$ is an extension of an abelian scheme by a torus, and hence the pull back of $\mathcal{H}_{1}\left(G^{\text {an }}, \mathbb{Z}\right)$ to $\left(\right.$ fsan $\left./\left(S^{\prime}\right)^{\text {an }}\right)$ is locally constant.

11.5.2. Locally on $S^{\text {an }}$, there is a locally constant subgroup sheaf $\left(X^{\prime}\right)^{*}$ of $\mathcal{H}_{1}\left(G^{\text {an }}, \mathbb{Z}\right)$ satisfying the following conditions (i)-(iii).

(i) The composition

$$
\mathbb{G}_{a} \otimes_{\mathbb{Z}}\left(X^{\prime}\right)^{*} \longrightarrow \mathbb{G}_{a} \otimes_{\mathbb{Z}} \mathcal{H}_{1}\left(G^{\mathrm{an}}, \mathbb{Z}\right) \longrightarrow \operatorname{Lie}\left(G^{\mathrm{an}}\right)
$$

is an isomorphism.

(ii) For each $s \in S^{\text {an }}$, the subgroup $H_{1}\left((T / s)^{\text {an }}, \mathbb{Z}\right)$ of $H_{1}\left(\left(G \times_{S} s\right)^{\text {an }}, \mathbb{Z}\right)$ is contained in the stalk of $\left(X^{\prime}\right)^{*}$ at $s$.

(iii) $\mathcal{H}_{1}\left(G^{\text {an }}, \mathbb{Z}\right) /\left(X^{\prime}\right)^{*}$ is torsion free.

Proof. For each $s \in S^{\text {an }}$, we can find such $\left(X^{\prime}\right)^{*}$ for the pull back of $A$ to $($ fsan $/ s)$. (The subgroup $\left(X^{\prime}\right)^{*}$ of $H_{1}\left(G^{\text {an }}, \mathbb{Z}\right)$ in $11.2(13)$ is fine.) For each $s,\left(X^{\prime}\right)^{*} \rightarrow \mathcal{H}_{1}\left(G^{\text {an }}, \mathbb{Z}\right)_{s}$ extends to $\left(X^{\prime}\right)^{*} \rightarrow \mathcal{H}_{1}\left(G^{\text {an }}, \mathbb{Z}\right)$ on an open neighborhood $U$ of $s$ in $S^{\text {an }}$. We show that the above (i)-(iii) are satisfied on an open neighborhood $V$ of $s$ in $U$.

The composition $\left.\mathcal{O}_{U} \otimes_{\mathbb{Z}}\left(X^{\prime}\right)^{*} \rightarrow \mathcal{O}_{U} \otimes_{\mathbb{Z}} \mathcal{H}_{1}\left(G^{\text {an }}, \mathbb{Z}\right) \rightarrow \operatorname{Lie}\left(G^{\text {an }}\right)\right|_{U}$ is a homomorphism of $\mathcal{O}_{U}$-modules which are locally free of finite rank and whose pull back to $s$ is an isomorphism. Hence it is an isomorphism on some open neighborhood of $s$ in $U$. We replace $U$ by this neighborhood.

Since $\left.\left(X^{\prime}\right)^{*} \rightarrow \mathcal{O}_{U} \otimes_{\mathbb{Z}}\left(X^{\prime}\right)^{*} \cong \operatorname{Lie}\left(G^{\text {an }}\right)\right|_{U}$ is injective and it factors through $\left(X^{\prime}\right)^{*} \rightarrow \mathcal{H}_{1}\left(G^{\text {an }}, \mathbb{Z}\right)$, the last map is injective.

We show that for some open neighborhood $V$ of $s$ in $U,\left(X^{\prime}\right)_{t}^{*}$ contains $H_{1}\left((T / t)^{\text {an }}, \mathbb{Z}\right)$ for any $t \in V$. The stalk $\xi_{s} \in \bar{X}_{s} \otimes H_{1}\left(\left(G \times_{S} s\right)^{\text {an }}, \mathbb{Z}\right)$ of $\xi$ belongs to $\bar{X}_{s} \otimes H_{1}\left((T / s)^{\text {an }}, \mathbb{Z}\right)(-1) \subset \bar{X}_{s} \otimes\left(X^{\prime}\right)_{s}^{*}(-1)$. Hence $\xi$ belongs to $\bar{X} \otimes\left(X^{\prime}\right)^{*}(-1)$ on some open neighborhood $V$ of $s$ in $U$ and hence induces 
a homomorphism $\operatorname{Hom}\left(\bar{X}_{t}, \mathbb{Z}\right)(1) \rightarrow\left(X^{\prime}\right)_{t}^{*}$ for each $t \in V$. For each $t \in V$, the diagram

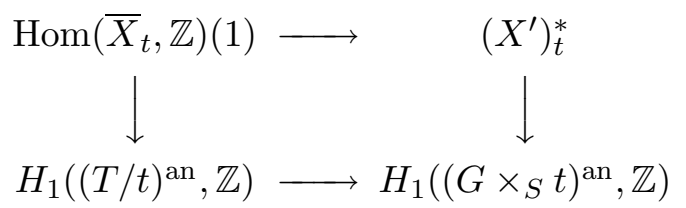

is commutative. Here the right vertical arrow and the lower horizontal arrow are inclusion maps and the other two arrows are induced by $\xi_{t}$. Since the left vertical arrow is an isomorphism, we have $H_{1}\left((T / t)^{\text {an }}, \mathbb{Z}\right) \subset\left(X^{\prime}\right)_{t}^{*}$.

Finally we prove that $\mathcal{H}_{1}\left(G^{\text {an }}, \mathbb{Z}\right) /\left(X^{\prime}\right)^{*}$ is torsion free on some open neighborhood of $s$ in $U$. By 11.5.1, if $W$ is a locally closed analytic subspace of $U$ such that $M_{W} / \mathcal{O}_{W}^{\times}$is locally constant, then the restriction of $\mathcal{H}_{1}\left(G^{\text {an }}, \mathbb{Z}\right) /\left(X^{\prime}\right)^{*}$ to $W$ is locally constant. Hence if we replace $U$ by a sufficiently small open neighborhood of $s$ in $U$, then the torsion part of $\mathcal{H}_{1}\left(G^{\text {an }}, \mathbb{Z}\right) /\left(X^{\prime}\right)^{*}$ is contained in the $n$-torsion part for some $n \geq 1$. The inclusion map $\left(X^{\prime}\right)^{*} \rightarrow \mathcal{H}_{1}\left(G^{\text {an }}, \mathbb{Z}\right)$ induces a surjective homomorphism $f: \mathcal{H o m}\left(X^{\prime}, \mathbb{G}_{m}\right) \rightarrow G^{\text {an }}$. We have an exact sequence

$$
0 \longrightarrow \mathcal{H}_{1}\left(G^{\text {an }}, \mathbb{Z}\right) /\left(X^{\prime}\right)^{*} \longrightarrow \mathcal{H o m}\left(X^{\prime}, \mathbb{G}_{m}\right) \stackrel{f}{\longrightarrow} G^{\text {an }} \longrightarrow 0 .
$$

Hence if we denote the $n$-torsion part of $\mathcal{H}_{1}\left(G^{\text {an }}, \mathbb{Z}\right) /\left(X^{\prime}\right)^{*}$ by $F$, we have the induced exact sequence

$$
0 \longrightarrow F \longrightarrow \mathcal{H o m}\left(X^{\prime}, \mu_{n}\right) \stackrel{f}{\longrightarrow} G^{\text {an }},
$$

where $\mu_{n}=\left\{z \in \mathbb{C}^{\times} \mid z^{n}=1\right\}$. For each non-trivial homomorphism $\psi: X^{\prime} \rightarrow \mu_{n}$, since $G$ is separated, the equalizer $C(\psi)$ of $f(\psi): U \rightarrow G^{\text {an }}$ and the unit section $U \rightarrow G^{\text {an }}$ is a closed analytic subspace of $U$. Since $F_{s}=0$, we have $s \notin C(\psi)$. Let $V=U \backslash \bigcup_{\psi} C(\psi)$. Then $V$ is an open neighborhood of $s$ in $U$, and $\left.F\right|_{V}=\{0\}$.

Working locally on $S^{\text {an }}$, let

$$
X^{\prime}=\mathcal{H o m}\left(\left(X^{\prime}\right)^{*}, \mathbb{Z}\right)(1)
$$

The section $\xi \in \bar{X} \otimes\left(X^{\prime}\right)^{*}(-1)$ defines a homomorphism $X^{\prime} \rightarrow \bar{X}$, which we still denote by $\xi$. At each $s \in S^{\text {an }}$, the stalk $\xi_{s}: X_{s}^{\prime} \rightarrow \bar{X}_{s}$ is the $\operatorname{Hom}(, \mathbb{Z}(1))$-dual of the inclusion map $H_{1}\left((T / s)^{\text {an }}, \mathbb{Z}\right) \rightarrow\left(X^{\prime}\right)_{s}^{*}$. Hence $\xi: X^{\prime} \rightarrow \bar{X}$ is surjective. 
From the pairing $X^{\prime} \times Y \rightarrow \bar{X} \times Y \rightarrow \mathbb{G}_{m, \log } / \mathbb{G}_{m}$ for which we have $\mathcal{H o m}\left(X^{\prime}, \mathbb{G}_{m, \log } / \mathbb{G}_{m}\right)^{(Y)}=\mathcal{H o m}\left(X, \mathbb{G}_{m, \log } / \mathbb{G}_{m}\right)^{(Y)}=L$, we obtain an exact sequence on $\left(\mathrm{fsan} / S^{\mathrm{an}}\right)$

$$
0 \longrightarrow \mathcal{H o m}\left(X^{\prime}, \mathbb{G}_{m}\right) \longrightarrow \mathcal{H o m}\left(X^{\prime}, \mathbb{G}_{m, \log }\right)^{(Y)} \longrightarrow L \longrightarrow 0 .
$$

Via the isomorphism $\mathcal{E} x t\left(L, \mathcal{H o m}\left(X^{\prime}, \mathbb{G}_{m}\right)\right) \cong \bar{X} \otimes\left(X^{\prime}\right)^{*}(-1)=\mathcal{H} \circ{ }^{\prime}\left(X^{\prime}\right.$, $\bar{X})$, this extension also corresponds to $\xi$. This fact with the commutative diagram

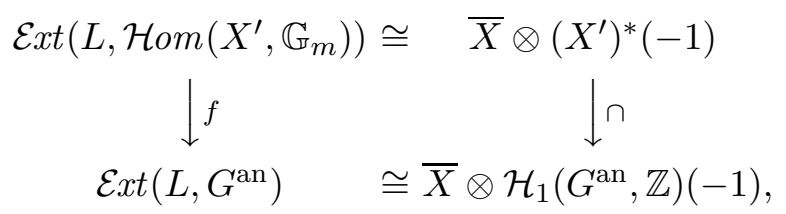

shows that the push forward of the exact sequence (2) by $f: \mathcal{H o m}\left(X^{\prime}, \mathbb{G}_{m}\right)$ $\rightarrow G^{\text {an }}$ coincides with the exact sequence (1). Hence we have a commutative diagram with exact rows

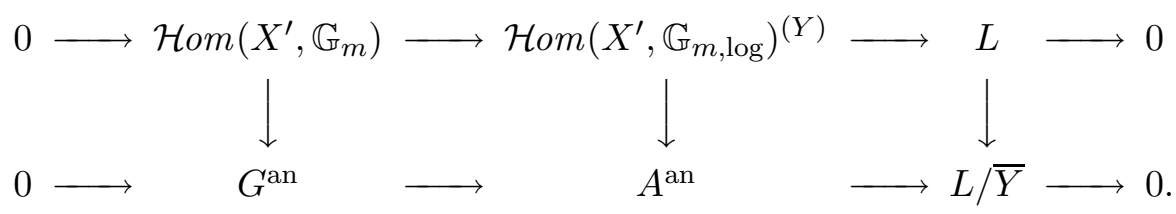

Let $Y^{\prime}$ be the kernel of the surjection $\mathcal{H o m}\left(X^{\prime}, \mathbb{G}_{m, \log }\right)^{(Y)} \rightarrow A^{\text {an }}$. The last commutative diagram gives the exact sequence of the kernels of the vertical arrows

$$
0 \longrightarrow \mathcal{H}_{1}\left(G^{\mathrm{an}}, \mathbb{Z}\right) / \mathcal{H o m}\left(X^{\prime}, \mathbb{Z}(1)\right) \longrightarrow Y^{\prime} \longrightarrow \bar{Y} \longrightarrow 0 .
$$

We have

$$
A^{\text {an }}=\mathcal{H o m}\left(X^{\prime}, \mathbb{G}_{m, \log }\right)^{(Y)} / Y^{\prime} .
$$

Hence Theorem 4.10 is proved if we prove

11.5.3. $Y^{\prime}$ is a locally constant sheaf.

We first prove

11.5.4. Let $W$ be an fs $\log$ analytic space over $S^{\text {an }}$ such that $M_{W} / \mathcal{O}_{W}^{\times}$ is locally constant. Then the pull back of $Y^{\prime}$ to $($ fsan $/ W)$ is locally constant.

This is proved in the same way as 11.5.1. As there, we are reduced to the case $M_{S} / \mathcal{O}_{S}^{\times}$is constant. Then $A$ is with constant degeneration, and hence $\mathcal{H}_{1}\left(G^{\text {an }}, \mathbb{Z}\right) / \mathcal{H o m}\left(X^{\prime}, \mathbb{Z}(1)\right)$ and $\bar{Y}$ are locally constant sheaves. Hence the exact sequence (3) shows that $Y^{\prime}$ is also locally constant.

From the exact sequence (3), we obtain also the following 11.5.5. 
11.5.5. Let $V$ be an fs $\log$ analytic space over $S^{\text {an }}$, let $v \in V$, and let $s \in S^{\text {an }}$ be the image of $v$. Then the map between the stalks $Y_{s}^{\prime} \rightarrow Y_{v}^{\prime}$ is bijective.

11.5.6. The stalks of $Y^{\prime}$ are finitely generated free $\mathbb{Z}$-modules. The $\mathbb{Z}$-rank of a stalk is a locally constant function on $S^{\text {an }}$ and is equal to the dimension of $A$.

Proof. Let $s \in S^{\text {an }}$. By the exact sequence (3), we have

$$
\operatorname{rank}_{\mathbb{Z}}\left(Y_{s}^{\prime}\right)=\operatorname{rank}_{\mathbb{Z}}\left(\bar{Y}_{s}\right)+\operatorname{rank}_{\mathbb{Z}}\left(\mathcal{H}_{1}\left(G^{\text {an }}, \mathbb{Z}\right)_{s}\right)-\operatorname{rank}_{\mathbb{Z}}\left(\left(X^{\prime}\right)_{s}^{*}\right) .
$$

We have $\operatorname{rank}_{\mathbb{Z}}\left(\bar{Y}_{s}\right)=\operatorname{dim}(T / s)$. Let $B / s$ be the quotient abelian variety $\left(G \times{ }_{S} s\right) /(T / s)$. Then

$$
\begin{aligned}
\operatorname{rank}_{\mathbb{Z}}\left(\mathcal{H}_{1}\left(G^{\text {an }}, \mathbb{Z}\right)_{s}\right) & =\operatorname{rank}_{\mathbb{Z}}\left(H_{1}\left((T / s)^{\text {an }}, \mathbb{Z}\right)\right)+\operatorname{rank}_{\mathbb{Z}}\left(H_{1}\left((B / s)^{\text {an }}, \mathbb{Z}\right)\right) \\
& =\operatorname{dim}(T / s)+2 \operatorname{dim}(B / s) .
\end{aligned}
$$

We have $\operatorname{rank}_{\mathbb{Z}}\left(\left(X^{\prime}\right)_{s}^{*}\right)=\operatorname{dim}\left(G \times_{S} s\right)$. These show that $\operatorname{rank}_{\mathbb{Z}}\left(Y_{s}^{\prime}\right)=$ $2 \operatorname{dim}(T / s)+2 \operatorname{dim}(B / s)-\operatorname{dim}\left(G \times \times_{S} s\right)=\operatorname{dim}\left(G \times{ }_{S} s\right)$.

11.5.7. $\mathbb{Q} \otimes Y^{\prime}$ is locally constant.

Proof. By 11.4.1, 11.4.3 and 11.5.5, we may assume that the underlying scheme of $S$ is smooth over $\mathbb{C}$ and the $\log$ structure of $S$ is $M_{S, D, r}$ (11.4.1) for some divisor $D$ on $S$ with normal crossings and for some $r \geq 0$.

Let $\Delta$ be the open unit disc $\{z \in \mathbb{C}|| z \mid<1\}$. Let $s \in S^{\text {an }}$. Then for some open neighborhood $U$ of $s$ in $S^{\text {an }}$, there is a homomorphism $h: Y_{s}^{\prime} \rightarrow$ $\left.Y^{\prime}\right|_{U}$ such that the composition $Y_{s}^{\prime} \rightarrow Y^{\prime}(U) \rightarrow Y_{s}^{\prime}$ is the identity map. We can take $U$ such that there is an isomorphism $U \cong \Delta^{a+b}(a, b \geq 0)$ which sends $s$ to the origin of $\Delta^{a+b}$ and which sends $U \cap D$ onto the subset of $\Delta^{a+b}$ consisting of all elements $\left(z_{i}\right)$ such that $z_{i}=0$ for some $1 \leq i \leq a$. It is sufficient to show that the restriction of $\mathbb{Q} \otimes Y^{\prime}$ to $U$ is constant. It is sufficient to prove that for any $t \in U, h$ induces an isomorphism $\mathbb{Q} \otimes Y_{s}^{\prime} \stackrel{\simeq}{\rightarrow} \otimes Y_{t}^{\prime}$. Since $\operatorname{rank}_{\mathbb{Z}}\left(Y_{s}^{\prime}\right)=\operatorname{rank}_{\mathbb{Z}}\left(Y_{t}^{\prime}\right)$ by 11.5.6, it is sufficient to prove that $Y_{s}^{\prime} \rightarrow Y_{t}^{\prime}$ is injective. Let $R$ be the sheaf of meromorphic functions on $S^{\text {an }}$. Since $Y^{\prime} \subset \mathcal{H o m}\left(X^{\prime}, \mathbb{G}_{m, \log }\right)$ and the restriction of $\mathbb{G}_{m, \log }$ to the topological space $S^{\text {an }}$ is a subsheaf of $\mathbb{N}^{r} \times R^{\times}$, and since the map $R(U) \rightarrow R_{t}$ is injective, the map $Y^{\prime}(U) \rightarrow Y_{t}^{\prime}$ is injective. As $Y_{s}^{\prime} \rightarrow Y^{\prime}(U)$ is clearly injective, we have the injectivity of $Y_{s}^{\prime} \rightarrow Y_{t}^{\prime}$.

To proceed from $\mathbb{Q} \otimes Y^{\prime}$ to $Y^{\prime}$, we use the following 11.5.8 and 11.5.9. 
11.5.8. Let $n \geq 1$ and let ${ }_{n} A=\operatorname{Ker}(n: A \rightarrow A)$. Let ${ }_{n} A_{s}$ be the stalk of the sheaf on the small étale site of $S$ defined by ${ }_{n} A$, and let ${ }_{n} A_{s}^{\text {an }}$ be the stalk at $s$ of the sheaf on $S^{\text {an }}$ defined by ${ }_{n} A^{\text {an }}$. Then the canonical map ${ }_{n} A_{s} \rightarrow{ }_{n} A_{s}^{\text {an }}$ is an isomorphism.

(Note that ${ }_{n} A_{s}$ is not ${ }_{n} A(s)$. If we denote by $\mathcal{O}_{S, s}^{h}$ the henselization of $\mathcal{O}_{S, s}$, then ${ }_{n} A_{s}={ }_{n} A\left(\mathcal{O}_{S, s}^{h}\right)$.)

Proof. We have a commutative diagram with exact rows

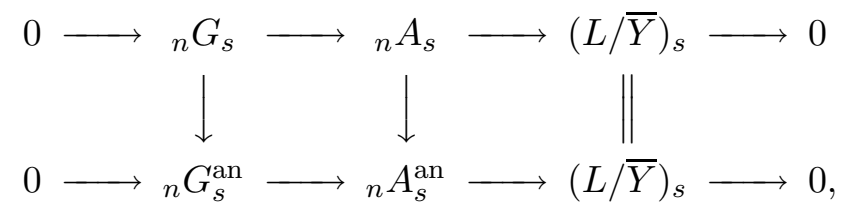

where $L=\mathcal{H o m}\left(X, \mathbb{G}_{m, \log } / \mathbb{G}_{m}\right)^{(Y)}$ (the algebraic version of this and the analytic one have the same stalks, as well as $\bar{Y}$ ) and the canonical map ${ }_{n} G_{s} \rightarrow{ }_{n} G_{s}^{\text {an }}$ is an isomorphism.

11.5.9. Let $U$ and $U \cong \Delta^{a+b}$ be as in the proof of 11.5.7. Let $t \in U$, and let $U(t)$ be the locally closed analytic subspace of $U$ defined to be the set of all $t^{\prime} \in U$ such that if $\left(t_{i}\right)$ (resp. $\left(t_{i}^{\prime}\right)$ ) denotes the image of $t$ (resp. $t^{\prime}$ ) in $\Delta^{a+b}$, then the sets $\left\{i \mid 1 \leq i \leq a, t_{i}=0\right\}$ and $\left\{i \mid 1 \leq i \leq a, t_{i}^{\prime}=0\right\}$ are the same. Endow $U(t)$ with the inverse image of the log structure of $S$. Then the restriction of $Y^{\prime}$ to $U(t)$ is locally constant.

Proof. Since $M_{U(t)} / \mathcal{O}_{U(t)}^{\times}$is locally constant, this follows from 11.5.4.

Now we prove that $Y^{\prime}$ is locally constant. It is sufficient to prove that for $U, s, t$ as above, the injection $h: Y_{s}^{\prime} \rightarrow Y_{t}^{\prime}$ is in fact an isomorphism. Note that to prove this, we may replace $S$ by any étale neighborhood $S^{\prime}$ of $s \rightarrow S, U$ by an open neighborhood $U^{\prime}$ of $s$ in $\left(S^{\prime}\right)^{\text {an }}$ contained in the inverse image of $U$ satisfying the same condition as $U$, and replace the point $t$ by any point $t^{\prime}$ of $U^{\prime}$ whose image in $U$ is contained in $U(t)$ (11.5.9).

Let $y \in Y_{t}^{\prime}$. We prove that $y$ belongs to the image of $Y_{s}^{\prime} \rightarrow Y_{t}^{\prime}$. There are $n \geq 1$ and $z \in Y_{s}^{\prime}$ such that $n y$ is the image of $z$.

We may assume that the following condition $(*)$ is satisfied.

(*) The image of the homomorphism $X^{\prime} \rightarrow M_{U, s}^{\mathrm{gp}}$ induced by $z$ is contained in the image of the $n$-th power map $n: M_{U, s}^{\mathrm{gp}} \rightarrow M_{U, s}^{\mathrm{gp}}$. 
In fact, as we have mentioned above, we can replace $S$ by an étale neighborhood of $s \rightarrow S$ on which the irreducible components of $D$ are defined by functions $f_{i}(1 \leq i \leq a)$. By 11.5.5, we can replace $S$ by the Kummer covering $S^{\prime}$ of $S$ defined by $n$-th roots of $f_{i}(1 \leq i \leq a)$ which we endow with the log structure replacing $\mathbb{N}^{r}$ by $((1 / n) \mathbb{N})^{r}$ and replacing $D$ by the inverse image of $D$ in $S^{\prime}$ which is still a normal crossing divisor, and replace $s$ by a point of $S^{\prime}$ lying over $s$. After these replacements, the condition $(*)$ is satisfied.

Assuming (*), take a homomorphism $\varphi: X^{\prime} \rightarrow M_{U, s}^{\mathrm{gp}}$ such that $n \varphi=z$. (We denote here the group law of $\mathcal{H o m}\left(X^{\prime}, \mathbb{G}_{m, \log }\right)$ additively). We may assume that $\varphi$ comes from a homomorphism $X^{\prime} \rightarrow M_{U}^{\mathrm{gp}}$ which we denote also by $\varphi$. We may further assume that the image of $n \varphi$ in $\operatorname{Hom}\left(X^{\prime}, M_{U, t}^{\mathrm{gp}}\right)$ coincides with $n y$ and hence the difference $y-\varphi$ at $t$ gives a homomorphism $\psi: X^{\prime} \rightarrow \mu_{n}$, where $\mu_{n}=\left\{z \in \mathbb{C}^{\times} \mid z^{n}=1\right\}$. Replacing $\varphi$ by $\varphi+\psi$, we obtain a homomorphism $\varphi: X^{\prime} \rightarrow M_{U}^{\mathrm{gp}}$ whose image in $\operatorname{Hom}\left(X^{\prime}, M_{U, t}^{\mathrm{gp}}\right)$ coincides with $y$. Let

$$
w=\left(\varphi \bmod Y^{\prime}\right) \in\left(\mathcal{H o m}\left(X^{\prime}, \mathbb{G}_{m, \log }\right)^{(Y)} / Y^{\prime}\right)(U)=A^{\text {an }}(U) .
$$

As $n \varphi \in Y^{\prime}, w$ belongs to ${ }_{n} A^{\text {an }}(U)$. We denote the image of $w$ under ${ }_{n} A_{s}^{\text {an }} \stackrel{\cong}{\rightrightarrows} A_{s}(11.5 .8)$ also by $w$. Replace $S$ by an étale neighborhood $S^{\prime}$ of $s \rightarrow S$ on which $w$ comes from an element of ${ }_{n} A\left(S^{\prime}\right)$ which we still denote by $w$. Let $E(w, 0)$ be the equalizer of $w: S \rightarrow A$ and the unit section $0: S \rightarrow A$.

Since the stalk of $\varphi$ at $t$ belongs to $Y_{t}^{\prime}$ and since $\left.Y^{\prime}\right|_{U(t)}$ is locally constant (11.5.9), the restriction of $\varphi$ on $U(t)$ belongs to $\left.Y^{\prime}\right|_{U(t)}$ and hence $w$ vanishes on $U(t)$. Hence the image of $E(w, 0)^{\text {an }} \rightarrow S^{\text {an }}$ contains $U(t)$. Since $E(w, 0)$ is finite over $S$, the image of $E(w, 0)^{\text {an }} \rightarrow S^{\text {an }}$ is closed. Since $s$ is contained in the closure of $U(t)$, the image of $E(w, 0)^{\text {an }} \rightarrow S^{\text {an }}$ contains $s$. Take $v \in E(w, 0)^{\text {an }}$ whose image in $S^{\text {an }}$ is $s$. Then $\varphi \in Y_{v}^{\prime}$ in $\mathbb{Q} \otimes Y_{v}^{\prime}$. Since $Y_{s}^{\prime}=Y_{v}^{\prime}(11.5 .5)$, we have $\varphi \in Y_{s}^{\prime}$ in $\mathbb{Q} \otimes Y_{s}^{\prime}$. Since the image of $\varphi$ in $Y_{t}^{\prime}$ is $y, y$ belongs to the image of $Y_{s}^{\prime} \rightarrow Y_{t}^{\prime}$.

This completes the proof of 11.5.3 and hence also the proof of Theorem 4.10 .

11.6. We show that if we drop the condition 4.1.3 in the definition of $\log$ abelian variety, then we would have a pathological object.

We consider an analytic object first. Let $n \geq 2$ and consider the exact sequence

$$
0 \longrightarrow \mathbb{Z} / n \mathbb{Z} \longrightarrow \mathbb{G}_{m, \log }^{(q)} / q^{n \mathbb{Z}} \longrightarrow \mathbb{G}_{m, \log }^{(q)} / q^{\mathbb{Z}} \longrightarrow 0
$$


on (fsan $/ \Delta)$, where $\Delta$ is the open unit disc endowed with the log structure associated to $\{0\} \subset \Delta$, and $q$ denotes the coordinate function of $\Delta$. Note that $\mathbb{G}_{m, \log }^{(q)} / q^{m \mathbb{Z}}$ for $m \geq 1$ are log abelian varieties over $\Delta$ in the sense of [6] (cf. 4.11.6). Define

$$
A=\left(\mathbb{G}_{m, \log }^{(q)} / q^{n \mathbb{Z}}\right) / j_{!}(\mathbb{Z} / n \mathbb{Z}),
$$

where $j$ is the inclusion map $\Delta \backslash\{0\} \rightarrow \Delta$. Then the pull back of $A$ to $\Delta \backslash\{0\}$ coincides with that of $\mathbb{G}_{m, \log }^{(q)} / q^{\mathbb{Z}}$, and the pull back of $A$ to $0 \in \Delta$ coincides with that of $\mathbb{G}_{m, \log }^{(q)} / q^{n \mathbb{Z}}$. This $A$ is not a log abelian variety over $\Delta$ in the sense of [6].

The above exact sequence comes from an exact sequence $0 \rightarrow \mathbb{Z} / n \mathbb{Z} \rightarrow$ $A_{1} \rightarrow A_{2} \rightarrow 0$ on a smooth curve $S$ over $\mathbb{C}$ with an fs log structure, where $A_{1}, A_{2}$ are $\log$ abelian varieties over $S$, via a morphism $\Delta \rightarrow S^{\text {an }}$. Let $s \in S$ be the image of $0 \in \Delta$ and let $j: S \backslash\{s\} \rightarrow S$ be the inclusion map. Then $A_{3}:=A_{1} / j_{!}(\mathbb{Z} / n \mathbb{Z})$ satisfies the conditions 4.1 .1 and 4.1.2 in the definition of $\log$ abelian variety, but not the condition 4.1.3. The pull back of $A_{3}$ to $\Delta$ is the above $A$. (The second condition is satisfied since if we denote by $G_{i}(i=1,2)$ the semi-abelian part of $A_{i}$, then we have an exact sequence $0 \rightarrow G_{2} \rightarrow A_{3} \rightarrow A_{1} / G_{1} \rightarrow 0$.)

In the proof of Theorem $4.10, Y^{\prime}$ for this object $A$ is not locally constant: The stalk of $Y^{\prime}$ at $0 \in \Delta$ is $q^{n \mathbb{Z}}$, but the stalks of $Y^{\prime}$ at points of $\Delta \backslash\{0\}$ are $q^{\mathbb{Z}}$.

\section{REFERENCES}

[1] P. Deligne and M. Rapoport, Les schemas de modules de courbes elliptiques, Modular functions of one variable, II, Antwerp 1972 Preceedings (P. Deligne and W. Kuyk, eds.), Lecture Notes in Math. 349, Berlin, Springer-Verlag, 1973, pp. 143-316.

[2] G. Faltings and C. Chai, Degeneration of abelian varieties, Ergebnisse der Mathematik und ihrer Grenzgebiete 3.Folge-Band 22, Springer-Verlag, Berlin, 1990.

[3] K. Fujiwara, Arithmetic compactifications of Shimura varieties (I), preprint (1990).

[4] A. Grothendieck and J. A. Dieudonne, Étude cohomologique des faisceaux cohérents (EGA III), Publ. Math., Inst. Hautes Étud. Sci. 11 (1961), 17 (1963).

[5] L. Illusie, An overview of the work of K. Fujiwara, K. Kato, and C. Nakayama on logarithmic étale cohomology, Cohomologies p-adiques et applications Arithmétiques (II) (P. Berthelot, J. M. Fontaine, L. Illusie, K. Kato and M. Rapoport, éd.), Astérisque 279, 2002, pp. 271-322.

[6] T. Kajiwara, K. Kato, and C. Nakayama, Logarithmic abelian varieties, Part I: Complex analytic theory, to appear in J. Math. Sci. Univ. Tokyo. 
[7] K. Kato, Logarithmic structures of Fontaine-Illusie, Algebraic analysis, geometry, and number theory (J.-I. Igusa, ed.), Johns Hopkins University Press, Baltimore, 1989, pp. 191-224.

[8] K. Kato, Toric singularities, Amer. J. Math., 116 (1994), 1073-1099.

[9] K. Kato and C. Nakayama, Log Betti cohomology, log étale cohomology, and log de Rham cohomology of log schemes over $\mathbb{C}$, Kodai Math. J., 22 (1999), 161-186.

[10] C. Nakayama, Logarithmic étale cohomology, Math. Ann., 308 (1997), 365-404.

[11] M. C. Olsson, Log algebraic stacks and moduli of log schemes, preprint.

[12] V. Pahnke, Uniformisierung log-abelscher Varietäten, Doctor thesis, Universität Ulm (2005).

[13] P. Roquette, Analytic theory of elliptic functions over local fields, Hamb. Math. Einzelschriften. Neue Folge·Heft 1, Vandenhoeck \& Ruprecht in Göttingen, 1970.

[14] J. H. Silverman, The arithmetic of elliptic curves, Graduate texts in mathematics; 106, Springer-Verlag, 1986.

Takeshi Kajiwara

Department of Applied Mathematics

Faculty of Engineering

Yokohama National University

Yokohama, 240-8501

Japan

kajiwara@ynu.ac.jp

Kazuya Kato

Department of Mathematics

Faculty of Science

Kyoto University

Kitashirakawa, Kyoto 606-8502

Japan

kzkt@math.kyoto-u.ac.jp

Chikara Nakayama

Department of Mathematics

Tokyo Institute of Technology

Ohokayama, Meguro, Tokyo 152-8551

Japan

cnakayam@math.titech.ac.jp 